University of Tennessee Health Science Center UTHSC Digital Commons

\title{
Dissecting the Novel Roles of TRIM56 in Antiviral Innate Immunity
}

Baoming Liu

University of Tennessee Health Science Center

Follow this and additional works at: https://dc.uthsc.edu/dissertations

Part of the Medical Immunology Commons

\section{Recommended Citation}

Liu, Baoming , "Dissecting the Novel Roles of TRIM56 in Antiviral Innate Immunity" (2016). Theses and Dissertations (ETD). Paper 375. http://dx.doi.org/10.21007/etd.cghs.2016.0394.

This Dissertation is brought to you for free and open access by the College of Graduate Health Sciences at UTHSC Digital Commons. It has been accepted for inclusion in Theses and Dissertations (ETD) by an authorized administrator of UTHSC Digital Commons. For more information, please contact jwelch30@uthsc.edu. 


\title{
Dissecting the Novel Roles of TRIM56 in Antiviral Innate Immunity
}

\begin{abstract}
The tripartite motif-containing (TRIM) proteins have emerged as a new class of host antiviral restriction factors, with several demonstrating roles in regulating innate antiviral responses. Of $>70$ known TRIMs, TRIM56 inhibits replication of bovine viral diarrhea virus, a ruminant pestivirus of the family Flaviviridae, but has no appreciable effect on VSV, a rhabdovirus. We have also shown that TRIM56 forms a complex with the Toll-like receptor-3 (TLR3) adaptor, TRIF, via its C-terminal residues 621-750, and augments TLR3-mediated interferon (IFN) induction and establishment of an antiviral state. Yet, TRIM56's antiviral spectrum and the precise underlying mechanisms by which TRIM56 executes its direct antiviral functions and modulates TLR3 signaling remain undefined. Also unclear are the molecular determinants governing the direct and indirect antiviral activities of TRIM56.
\end{abstract}

Herein, in Chapter 3, I show that TRIM56 poses a barrier to infections by yellow fever virus (YFV), dengue virus serotype-2 (DENV2), and human coronavirus virus (HCoV)-OC43. Moreover, I demonstrate that TRIM56's anti-flavivirus effects required both the E3 ligase activity that lies in the N-terminal RING domain and the integrity of its C-terminal portion, while the restriction of HCoV-OC43 relied upon the TRIM56 E3 ligase activity alone. Furthermore, TRIM56 was revealed to impair YFV and DENV2 propagation by suppressing intracellular viral RNA accumulation but to compromise HCoV-OC43 infection at a later step in the viral life cycle, suggesting that distinct TRIM56 domains accommodate differing antiviral mechanisms. Next, in Chapter 4, I show TRIM56 puts a check on replication of influenza A and B viruses in cell culture. Interestingly, the anti-influenza activity was independent of the E3 ligase activity, B-box, or coiled-coil domains. Rather, deletion of a 63-residue long, C-terminal tail portion of TRIM56 abrogated the antiviral function. Moreover, expression of this short C-terminal segment curtailed the replication of influenza viruses as effectively as that of full-length TRIM56. Mechanistically, TRIM56 was found to specifically impede intracellular influenza virus RNA synthesis. Altogether, TRIM56 is a versatile antiviral host factor that confers resistance to YFV, DENV2, HCoV-OC43 and influenza viruses through overlapping and distinct molecular determinants. Last, in Chapter 5, I report TRIM56 over-expression promoted activation of NF-KB following TLR3 engagement but not that induced by TNF- $\alpha-$ or IL-1 $\beta$. Next, I observed that the coiled-coil domain and residues 431-610, but not the B-box or residues 355-433, were required for TRIM56 augmentation of TLR3-dependent IFN- $\beta$ promoter activation. Furthermore, alanine screening mutagenesis suggested the S469A+S471A+S475A triple mutant and S471A, S475A and S710A single mutant failed to enhance TLR3 signaling. In line with this, S471A, S475A and S710A, as well as the coiledcoil deletion mutant lost the ability to enhance poly-l:C-mediated establishment of an antiviral state compared with wild-type TRIM56. Collectively, these data reveal novel insights into the mechanism of TRIM56 augmentation of TLR3-dependent antiviral response and highlight a role for TRIM56 scaffolding and phosphorylation in positive regulation of TLR3 signaling.

\author{
Document Type \\ Dissertation \\ Degree Name \\ Doctor of Philosophy (PhD) \\ Program \\ Biomedical Sciences \\ Research Advisor \\ Kui Li, Ph.D.
}




\section{Keywords}

Antiviral, dengue, influenza, innate immunity, TRIM, virus

\section{Subject Categories}

Medical Immunology | Medical Sciences | Medicine and Health Sciences 
Dissecting the Novel Roles of TRIM56 in Antiviral Innate Immunity

\author{
A Dissertation \\ Presented for \\ The Graduate Studies Council \\ The University of Tennessee \\ Health Science Center
}

\author{
In Partial Fulfillment \\ Of the Requirements for the Degree \\ Doctor of Philosophy \\ From The University of Tennessee
}

By

Baoming Liu

May 2016 
Portions of Chapters 2 and 3 (C) 2014 by American Society for Microbiology. Portions of Chapters 2 and 4 (C) 2016 by American Society for Microbiology. All other material (C) 2016 by Baoming Liu. All rights reserved. 


\section{ACKNOWLEDGEMENTS}

I would like to thank my graduate advisor, Kui Li, Ph.D., and my advisory committee: Meiyun Fan, Ph.D., Lorraine M. Albritton, Ph.D., Richard Webby, Ph.D., and Tony N. Marion, Ph.D., for their support and helpful advice. I would also like to acknowledge previous and current lab members -- Nan L. Li, M.D., Jie Wang, Ph.D., Dahai Wei, Ph.D., Yang Shen, Ph.D., Kattareeya Kumthip, Ph.D., Xiaofan Li, Ph.D., and Sandra Lester, Ph.D., for their comments and discussion on my work. I appreciate Mark Zanin, Ph.D. at St. Jude Children's Research Hospital and Michelle Sims at University of Tennessee Health Science Center for assistance with confocal microscopy. Additionally, I would like to recognize all the faculty and staff of the University of Tennessee Health Science Center Department of Microbiology, Immunology and Biochemistry for creating an excellent atmosphere and environment for learning and performing research; in particular Michael Whitt, Ph.D., Elizabeth Fitzpatrick, Ph.D., and David Nelson, Ph.D. for their suggestions on course study, and John Cox, Ph.D. for microscopy assistance.

This research was supported by National Institutes of Health grants AI101526 and AI069285 from the National Institute of Allergy and Infectious Diseases to K.L. as well as Frank Dugan Scholarship, American Heart Association Predoctoral Fellowship 15PRE25080234 and Sigma Xi Grant-in-Aid of Research awards G20141015598932 and G201510151400090 to B.L. 


\begin{abstract}
The tripartite motif-containing (TRIM) proteins have emerged as a new class of host antiviral restriction factors, with several demonstrating roles in regulating innate antiviral responses. Of $>70$ known TRIMs, TRIM56 inhibits replication of bovine viral diarrhea virus, a ruminant pestivirus of the family Flaviviridae, but has no appreciable effect on VSV, a rhabdovirus. We have also shown that TRIM56 forms a complex with the Tolllike receptor-3 (TLR3) adaptor, TRIF, via its C-terminal residues 621-750, and augments TLR3-mediated interferon (IFN) induction and establishment of an antiviral state. Yet, TRIM56's antiviral spectrum and the precise underlying mechanisms by which TRIM56 executes its direct antiviral functions and modulates TLR3 signaling remain undefined. Also unclear are the molecular determinants governing the direct and indirect antiviral activities of TRIM56.

Herein, in Chapter 3, I show that TRIM56 poses a barrier to infections by yellow fever virus (YFV), dengue virus serotype-2 (DENV2), and human coronavirus virus (HCoV)-OC43. Moreover, I demonstrate that TRIM56's anti-flavivirus effects required both the E3 ligase activity that lies in the N-terminal RING domain and the integrity of its C-terminal portion, while the restriction of $\mathrm{HCoV}-\mathrm{OC} 43$ relied upon the TRIM56 E3 ligase activity alone. Furthermore, TRIM56 was revealed to impair YFV and DENV2 propagation by suppressing intracellular viral RNA accumulation but to compromise $\mathrm{HCoV}-\mathrm{OC} 43$ infection at a later step in the viral life cycle, suggesting that distinct TRIM56 domains accommodate differing antiviral mechanisms. Next, in Chapter 4, I show TRIM56 puts a check on replication of influenza A and B viruses in cell culture. Interestingly, the anti-influenza activity was independent of the E3 ligase activity, B-box, or coiled-coil domains. Rather, deletion of a 63-residue long, C-terminal tail portion of TRIM56 abrogated the antiviral function. Moreover, expression of this short C-terminal segment curtailed the replication of influenza viruses as effectively as that of full-length TRIM56. Mechanistically, TRIM56 was found to specifically impede intracellular influenza virus RNA synthesis. Altogether, TRIM56 is a versatile antiviral host factor that confers resistance to YFV, DENV2, HCoV-OC43 and influenza viruses through overlapping and distinct molecular determinants. Last, in Chapter 5, I report TRIM56 over-expression promoted activation of NF- $\kappa \mathrm{B}$ following TLR3 engagement but not that induced by TNF- $\alpha$ - or IL-1 $\beta$. Next, I observed that the coiled-coil domain and residues 431-610, but not the B-box or residues 355-433, were required for TRIM56 augmentation of TLR3-dependent IFN- $\beta$ promoter activation. Furthermore, alanine screening mutagenesis suggested the S469A+S471A+S475A triple mutant and S471A, S475A and S710A single mutant failed to enhance TLR3 signaling. In line with this, S471A, S475A and S710A, as well as the coiled-coil deletion mutant lost the ability to enhance poly-I:Cmediated establishment of an antiviral state compared with wild-type TRIM56. Collectively, these data reveal novel insights into the mechanism of TRIM56 augmentation of TLR3-dependent antiviral response and highlight a role for TRIM56 scaffolding and phosphorylation in positive regulation of TLR3 signaling.
\end{abstract}




\section{TABLE OF CONTENTS}

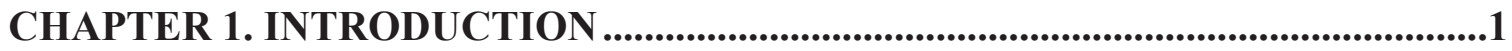

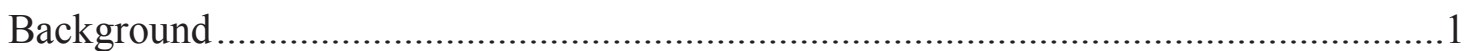

TLR3-mediated Innate Immune Responses against Positive-strand RNA Viruses.....1

Host Responses to Influenza Virus Infection...............................................................

TRIMs: Novel Players in Antiviral Innate Immunity .................................................

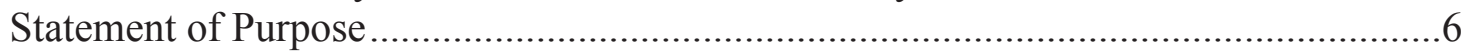

CHAPTER 2. METHODS AND MATERIALS.......................................................

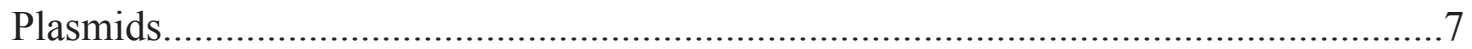

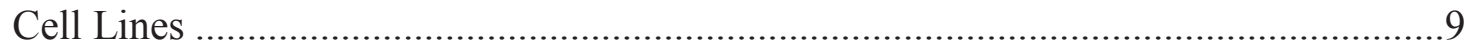

Viruses, Viral Infections, Replication Assays and Miscellaneous Reagents .................10

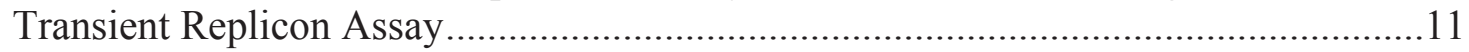

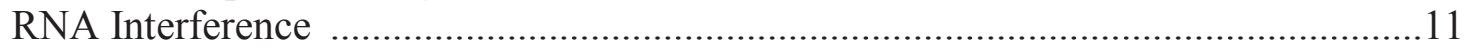

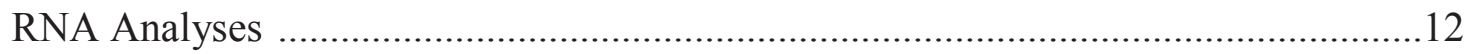

Luciferase Reporter-based Minigenome Replication Assay of IAV RNA Synthesis ..13

Immunoblotting, Immunofluorescence Staining, and Confocal Microscopy ................13

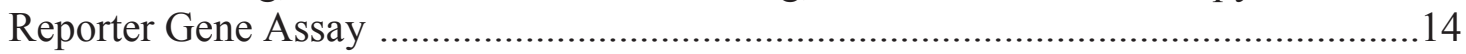

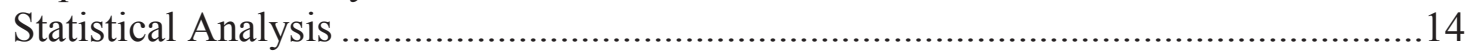

CHAPTER 3. OVERLAPPING AND DISTINCT MOLECULAR DETERMINANTS DICTATING THE ANTIVIRAL ACTIVITIES OF TRIM56 AGAINST FLAVIVIRUSES AND CORONAVIRUS.................................................15

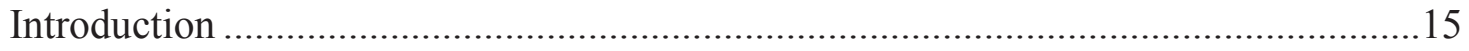

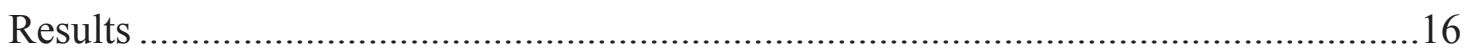

Ectopic Expression of TRIM56 Inhibits Propagation of YFV, DENV2 and

HCoV-OC43, but Not That of EMCV …………………..................................... 16

TRIM56 Expressed at Physiologic Levels Restricts Infections by YFV, DENV2

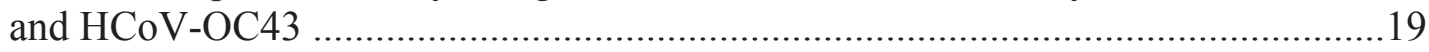

The Antiviral Effects of TRIM56 Are Not Attributed to General Augmentation

of IFN Response or to Regulation of Cell Growth .................................................21

TRIM56's Antiviral Actions Do Not Depend upon TLR3/TRIF, RIG-I or

STING

Development of HEK293 Cells with Conditional Expression of Various

TRIM56 Mutants for Mapping of the Antiviral Determinants

The E3 Ligase Activity and the Integrity of the C-terminus of TRIM56 Are

Critical for the Antiviral Activity against YFV and DENV2, while the B-box Is

Dispensable

The Functional TRIM56 Mutants Exert Their Antiviral Effects Independent of

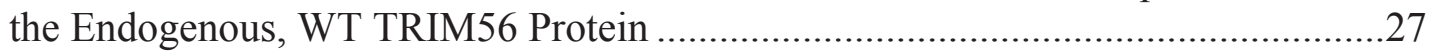

TRIM56's Suppression of HCoV-OC43 Multiplication Depends on Its E3

Ligase Activity, but Not the Integrity of the C-terminus or B-box or Coiled-coil Domains 
TRIM56 Inhibits YFV/DENV2 Propagation by Impairing Intracellular Viral RNA Replication, whereas It Curbs HCoV-OC43 Spread by Targeting a Later

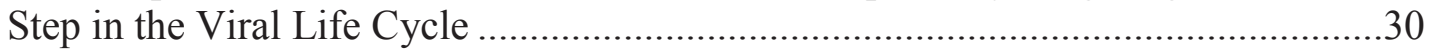

Discussion

\section{CHAPTER 4. THE C-TERMINAL TAIL OF TRIM56 DICTATES ANTIVIRAL RESTRICTION OF INFLUENZA A AND B VIRUSES BY IMPEDING VIRAL RNA SYNTHESIS..}

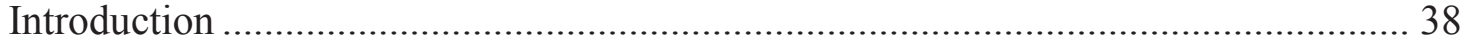

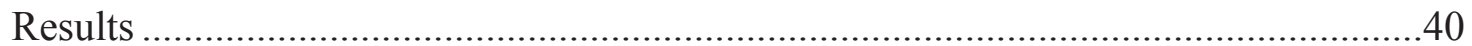

Ectopic Expression of TRIM56 Inhibits Propagation of IAV and IBV, but Not

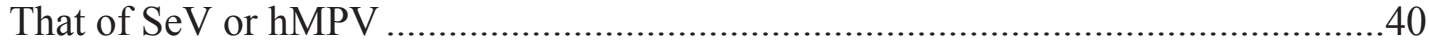

Knockdown of TRIM56 Augments Multiplication of Influenza Viruses.................43

The C-terminal Tail of TRIM56, but Not Its E3 Ligase Activity or the Other

Portions of the Protein, Is Required for the Ability to Impede Influenza Virus

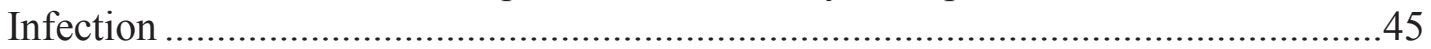

TRIM56's C-terminal Tail Is Sufficient to Curb Influenza Virus Infection..............48

TRIM56 Curtails Influenza Virus Propagation by Targeting Virus RNA

Synthesis

The C-terminal Tail of TRIM56 Is Both Required and Sufficient for the

Suppression of Influenza Virus RNA Synthesis .................................................51

TRIM56 Moves into the Nucleus during Influenza Infection...............................53

Discussion

\section{CHAPTER 5. MOLECULAR DETERMINANTS GOVERNING TRIM56- MEDIATED POSITIVE REGULATION OF THE TLR3 ANTIVIRAL SIGNALING PATHWAY}

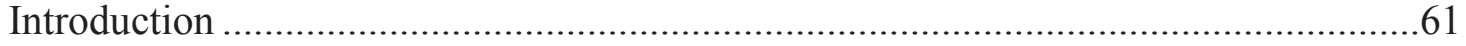

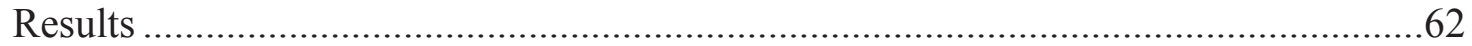

TRIM56 Promotes TLR3-mediated, IRF3- or NF-кB-dependent Signaling

Pathway via Forming a Complex with TRIF

The Coiled-coil Domain and Residues 431-610 of TRIM56, but Not B-box or

Residues 355-433, Are Critical for Enhancing TLR3-mediated IRF3- or NF-кB-

dependent Signaling

Putative Phosphorylation Sites S471, S475 and S710 Are Required for TRIM56-

mediated Positive Regulation of TLR3 Signaling

Alanine Substitutions on the Important TRIM56's Putative Phosphorylation

Sites and Deletion of the Coiled-coil Domain Undermine the TRIM56-mediated

Enhancement of TLR3-dependent Antiviral Response

TRIM56's Putative Phosphorylation Site S710 on the C-terminus, but Not the

Coiled-coil Domain, Is Critical for Its Association with TRIF 75

Discussion 
CHAPTER 6. DISCUSSION .

The Distinct Domain Requirements for TRIM56's Anti-flaviviruses and AntiHCoV-OC43 Actions May Reflect Different Molecular Mechanisms That TRIM56

Adopts to Restrict Different Positive-strand RNA Viruses.

Possible Molecular Mechanisms Underlying Which C-terminal End of TRIM56

Undermines Influenza Virus RNA Replication. .80

Dissect the Molecular Mechanisms Dictating TRIM56-mediated Positive

Regulation of the TLR3 Signaling Pathway.

Conclusion

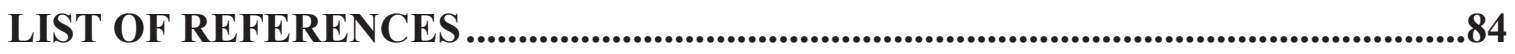

VITA 


\section{LIST OF TABLES}

Table 2-1. Mutagenesis primers for human (hT56) or mouse (mT56) TRIM56 ............8 


\section{LIST OF FIGURES}

Figure 1-1. TRIM family members and their subfamily classification ..........................4

Figure 1-2. Roles of TRIMs in innate antiviral immunity............................................5

Figure 3-1. Ectopic expression of TRIM56 inhibits multiplication of YFV, DENV2 and $\mathrm{HCoV}-\mathrm{OC} 43$, but not that of EMCV

Figure 3-2. The endogenous TRIM56 protein restricts propagation of YFV, DENV2 and $\mathrm{HCoV}-\mathrm{OC} 43$.

Figure 3-3. The antiviral effects of TRIM56 do not result from up-regulation of IFN response or from modulation of cell growth

Figure 3-4. TRIM56's antiviral actions are independent of TRIF, RIG-I and STING ...23

Figure 3-5. Characterization of HEK293 cells that conditionally express individual TRIM56 mutants in a tet-inducible manner

Figure 3-6. The E3 ligase activity and the integrity of the C-terminal parts of TRIM56 are essential for the antiviral activity against YFV

Figure 3-7. The suppression of DENV2 multiplication by TRIM56 requires its E3 ligase activity and the integrity of the C-terminal portions

Figure 3-8. Knockdown of endogenous TRIM56 via an siRNA targeting the 3' UTR of human TRIM56 transcript does not affect the antiviral activities of the ectopically expressed WT TRIM56 or functional TRIM56 mutants .....29

Figure 3-9. The inhibition of HCoV-OC43 propagation by TRIM56 is dependent upon its E3 ligase activity, but not upon the integrity of the $\mathrm{C}$-terminal portions, or the B-box or coiled-coil domains.

Figure 3-10. TRIM56 blunts YFV and DENV2 infection by targeting intracellular viral RNA replication, but inhibits $\mathrm{HCoV}-\mathrm{OC} 43$ propagation by acting on a later step in the viral life cycle.

Figure 4-1. Ectopic expression of TRIM56 hinders propagation of IAV/IBV, but not infection by hMPV or Sendai virus

Figure 4-2. TRIM56 knockdown augments influenza virus multiplication

Figure 4-3. The restriction of IAV by TRIM56 relies on its $\mathrm{C}$-terminal tail portion, but not on its E3 ligase activity or other parts of the protein . 
Figure 4-4. TRIM56's C-terminal tail is the only prerequisite for its anti-IBV function

Figure 4-5. Expression of TRIM56's C-terminal tail alone is sufficient to curb infection by IAV or IBV

Figure 4-6. TRIM56 hinders IAV propagation by inhibiting intracellular virus RNA synthesis

Figure 4-7. The C-terminal tail portion is required for TRIM56 suppression of influenza virus RNA synthesis....

Figure 4-8. TRIM56 and TRIM56-CTT63 move into the nucleus after IAV infection ..55

Figure 4-9. Sequence alignment of the C-terminal portion of TRIM56 with the NHL repeats of well-characterized TRIM-NHL proteins

Figure 5-1. TRIM56 positively regulates TLR3-mediated IRF3- or NF-кBdependent signaling pathway by forming a complex with TRIF

Figure 5-2. TRIM56's coiled-coil domain and residues 431-610, but not the B-box or residues 355-433, are required for its augmentation of TLR3mediated IRF3-dependent signaling pathway

Figure 5-3. TRIM56's coiled-coil domain and residues 431-610, but not the B-box or residues 355-433, are critical for its potentiation of TLR3-mediated $\mathrm{NF}-\kappa \mathrm{B}-\mathrm{dependent} \mathrm{signaling} \mathrm{pathway.}$

Figure 5-4. Putative phosphorylation on TRIM56's C-terminal serines S471, S475 and S710 is essential for its positive regulation of TLR3-mediated signaling pathway

Figure 5-5. TRIM56's coiled-coil domain and C-terminal putative phosphorylation sites S710, S471 and S475 are important for the establishment of an antiviral state by TLR3 ligand....

Figure 5-6. TRIM56's putative phospho-site S710, but not its coiled-coil domain, is important for its interaction with TRIF .76 


\section{LIST OF ABBREVIATIONS}

\begin{tabular}{|c|c|}
\hline aa & amino acids \\
\hline boT56 & bovine TRIM56 \\
\hline BVDV & bovine viral diarrhea virus \\
\hline $\mathrm{CPE}$ & cytopathic effect \\
\hline Ctrl & control \\
\hline CTT & C-terminal-tail \\
\hline CVD & cardiovascular disease \\
\hline DENV & dengue virus \\
\hline dsDNA & double-strand DNA \\
\hline dsRNA & double-strand RNA \\
\hline EMCV & encephalomyocarditis virus \\
\hline FIT & Flp-In T-REx \\
\hline GFP & green fluorescent protein \\
\hline $\mathrm{HCoV}$ & human coronavirus \\
\hline $\mathrm{HCV}$ & hepatitis $\mathrm{C}$ virus \\
\hline HEK & human embryo kidney \\
\hline HIV & human immunodeficiency virus \\
\hline hMPV & human metapneumovirus \\
\hline hpi & hours post infection \\
\hline hpt & hours post transfection \\
\hline hT56 & human TRIM56 \\
\hline IAV & influenza $A$ virus \\
\hline IBV & influenza B virus \\
\hline IFN & interferon \\
\hline IKK & I $\kappa \mathrm{B}$ kinase \\
\hline IL & interleukin \\
\hline IL-1R & interleukin-1 receptor \\
\hline IRF & interferon regulatory factor \\
\hline ISG & IFN-stimulated gene \\
\hline luc & luciferase \\
\hline M & matrix \\
\hline $\mathrm{mAb}$ & monoclonal antibody \\
\hline MDBK & Madin-Darby bovine kidney \\
\hline MDCK & Madin-Darby canine kidney \\
\hline MLV & murine leukemia virus \\
\hline MMP & matrix metalloproteinase \\
\hline MOI & multiplicity of infection \\
\hline mT56 & murine TRIM56 \\
\hline Mut & mutant \\
\hline MyD88 & myeloid differentiation primary response 88 \\
\hline $\mathrm{NF}-\kappa \mathrm{B}$ & nuclear factor kappa-light-chain-enhancer of activated B cells \\
\hline NHL & NCL-1-HT2A-LIN-41 \\
\hline NLS & nuclear localization sequence \\
\hline
\end{tabular}




$\begin{array}{ll}\text { NP } & \text { Nucleoprotein } \\ \text { NS } & \text { non-structural } \\ \text { pAb } & \text { polyclonal antibody } \\ \text { PAMP } & \text { pathogen-associated molecular pattern } \\ \text { pIC } & \text { poly-I:C } \\ \text { PRR } & \text { pattern recognition receptor } \\ \text { qPCR } & \text { quantitative PCR } \\ \text { RBCC } & \text { RING-B box-Coiled coil } \\ \text { RIG-I } & \text { retinoic-inducible gene-I } \\ \text { SeV } & \text { Sendai virus } \\ \text { shRNA } & \text { short hairpin RNA } \\ \text { siRNA } & \text { small interfering RNA } \\ \text { STING } & \text { stimulator of interferon genes } \\ \text { TBEV } & \text { tick-borne encephalitis virus } \\ \text { TCID } 50 & \text { 50\% tissue culture infective dose } \\ \text { tet } & \text { Tetracycline } \\ \text { TIR } & \text { Toll-interleukin-1 receptor } \\ \text { TLR } & \text { Toll-like receptor } \\ \text { TRIF } & \text { TIR-domain-containing adaptor inducing interferon- } \beta \\ \text { TRIM } & \text { tripartite motif-containing protein } \\ \text { Ub } & \text { Ubiquitin } \\ \text { UTR } & \text { untranslated region } \\ \text { vRNA } & \text { viral RNA } \\ \text { vRNP } & \text { viral ribonucleoprotein } \\ \text { VSV } & \text { vesicular stomatitis virus } \\ \text { WT } & \text { wild type } \\ \text { YFP } & \text { yellow fluorescent protein } \\ \text { YFV } & \text { yellow fever virus } \\ & \end{array}$




\section{CHAPTER 1. INTRODUCTION}

\section{Background}

\section{TLR3-mediated Innate Immune Responses against Positive-strand RNA Viruses}

In response to viral invasion, the innate immune system plays a critical role in recognizing intruding viral pathogens and inducing antiviral or inflammatory responses, thereby facilitating adaptive immune responses (1-4). Among the multilayered mechanisms of host intrinsic defense, germline-encoded pattern recognition receptors (PRRs) present on innate immune sentinel cells, such as Toll-like receptors (TLRs) and retinoic-inducible gene-I (RIG-I)-like receptors, play important parts in sensing viral nucleic acids or replicative intermediates produced during viral replication as a major class of viral pathogen-associated molecular pattern (PAMP). Those receptors initiate downstream intracellular signaling cascades that culminate in the production of interferons (IFNs) and pro-inflammatory cytokines/chemokines. Therefore, the innate immune responses constitute a primary and fundamental component of host defense in the immediate-early phase of viral infections (2-5).

Among various host PRRs, TLRs represent the first type to be discovered and characterized. There are 10 human TLRs (TLR1-TLR10) and 12 murine TLRs (TLR1-9 and TLR11-13) (4). Structurally speaking, all TLRs are type I transmembrane proteins consisting of N-terminal leucine-rich repeat-containing ectodomain which interacts with and recognizes PAMPs, a transmembrane domain, and a C-terminal Toll-interleukin-1 receptor (IL-1R) homology (TIR) domain which is responsible for activation of signaling transduction via interacting with their adaptor proteins $(1,2,4)$. There are only two types of adaptors for TLRs-mediated signal transduction - either myeloid differentiation primary response 88 (MyD88) or TIR-domain-containing adaptor inducing interferon- $\beta$ (TRIF) (4). Remarkably, TLR3 only signals through TRIF while the other TLRs signal through MyD88, with TLR4 using both adaptors. TLRs also vary on cellular localization and the ligands (PAMPs) to be sensed (4). For example, TLR2 and TLR4 on the cell surface are responsible for recognition of viral proteins. TLR3, TLR7, TLR8 and TLR9 localized in endosomes sense intracellular viral nucleic acids (1). Specifically, TLR3, TLR7/TLR8 and TLR9 recognize viral dsRNA, ssRNA and unmethylated CpG DNA, respectively. Engagement of TLRs with their cognate ligands activates distinct, intracellular signaling pathways through the adaptor proteins, which relay signals to downstream IאB (IKK) and IKK-related kinases (2-4). Subsequently, the classical IKKs $(\mathrm{IKK} \alpha / \mathrm{IKK} \beta / \mathrm{IKK} \gamma)$ activate the transcription factor NF- $\kappa \mathrm{B}$ for downstream proinflammatory cytokine production, while the IKK-related kinases (TBK1/IKKe) phosphorylate and activate IFN-regulatory factors IRF3 and/or IRF7 which are critical for induction of type I IFNs (IFN- $\beta$ and IFN- $\alpha$ ). IFNs further induce hundreds of IFNstimulated genes (ISGs) to establish an antiviral state restricting viral propagation and dissemination (1-4). 
Among different TLRs, TLR3 represents the first one to be well characterized which senses nucleic acid, i.e., dsRNA, and TLR3 as an essential PRR has been demonstrated to play important roles in sensing and fending off positive-strand RNA viruses $(1,2,4)$. For instance, TLR3 is able to sense the infection by dengue virus and plays a protective role against West Nile Virus infection (6,7). TLR3 signaling also has a protective effect on the heart after encephalomyocarditis virus-induced injury and decreases the virulence of this picornavirus (8). However, facing the host innate immune system, numerous viruses have evolved to acquire different strategies to circumvent or counteract with the TLRdependent host defense responses (4). For instance, vaccinia viruses encode two proteins, A46R and A52R, which associate with TLR adaptor proteins and TRAF6, respectively, thereby blocking TLR signaling $(9,10)$. Another appealing example is hepatitis $\mathrm{C}$ virus which utilizes its NS3/4A serine protease to cleave TRIF, the sole adaptor for TLR3, to compromise TLR3-mediated host antiviral immunity (11).

\section{Host Responses to Influenza Virus Infection}

Classified within the family Orthomyxoviridae, influenza A and B viruses (IAV and IBV) are important respiratory pathogens responsible for significant morbidity and mortality worldwide (12-15). Apart from typical influenza diseases, influenza virus infection may trigger severe influenza-related complications, for instance, cardiovascular diseases (CVDs) $(12,13)$. This is especially true for the elderly who represents an overlapping high-risk population for both influenza virus infections and CVDs (16-18). Remarkably, epidemiological studies have established an association of IAV infection with CVDs (16-18). Influenza viruses primarily infect lungs and respiratory tract but can trigger cardiac complications, such as atherosclerosis and acute myocardial infarction (16-18). Influenza vaccines are commonly considered to be an effective measure to prevent influenza infection. However, despite its protective effects shown on some CVD patients, influenza vaccine efficacy declines with age: from approximately $59 \%$ in adults aged $18-65$ years to $49 \%$ in the $65+$ population (19). What's worse, the elderly with underlying CVDs are particularly vulnerable to unanticipated new influenza subtypes that emerge. These issues underscore the importance of developing novel and broad-spectrum anti-influenza approaches to provide high-risk populations with better CVD prevention $(18,19)$.

Influenza viruses are characterized by segmented, negative-strand RNA genomes in the viral particle core, which are encapsidated by nucleoprotein (NP) and bound with polymerase proteins, thus forming viral ribonucleoprotein (vRNP) complexes $(15,20,21)$. Influenza virus life cycles are featured by the dependence on nuclear functions, which is rare to RNA viruses (15). After cell entry, trafficking of the viral genome into the nucleus is an active process dependent on the nuclear import machinery. Although the major viral components of IAV vRNP complexes all possess nuclear localization sequences (NLSs), the signals on NP are believed to be both sufficient and necessary for recognition and pick-up by cellular importins $(15,20,21)$. Among the localization signals, the strongest unconventional NLS-1 of NP is found at its extreme N-terminus, a weaker, bipartite NLS-2 is present in the middle of NP (residues 198-216), and a nuclear accumulation 
sequence (NAS) has been detected at the C-terminus (residues 336-345). These NLSs on NP are also important for nuclear import of this viral protein after translation $(15,20,21)$.

In response to influenza invasion, the innate immune system represents a front line of host defense armed with multi-layered mechanisms. Of these, PRRs such as TLRs and RIG-I-like receptors, sense viral nucleic acids, a major class of viral PAMPs triggering intracellular signaling pathways that activate IRF3 and NF- $\kappa \mathrm{B}$, which play pivotal roles in the production of IFNs and pro-inflammatory cytokines/chemokines (22-24). Influenza virus, in return, inhibits IRF3 activation by its non-structural protein (NS)-1 to decrease IFN generation whereas it exploits activated NF- $\kappa B$ to regulate its viral RNA (vRNA) synthesis, develop inflammation and induce synthesis of ectopic trypsins and matrix metalloproteinases (MMPs), thereby triggering cardiac injuries (22-26). IAV structural proteins, e.g. NP, can also augment NF- $\mathrm{B}$ signaling, the molecular mechanisms underlying which remain elusive (27).

\section{TRIMs: Novel Players in Antiviral Innate Immunity}

The tripartite motif-containing (TRIM) protein family consists of more than 70 members in human beings. It is previously known that TRIMs play essential roles in a broad array of biological processes, including proliferation, differentiation and apoptosis. Over the past decade, people have begun to appreciate important roles of TRIM family members in antiviral innate immunity (28-30). As suggested by their names, there are three highly conserved domains in TRIMs' N-terminal RBCC motif, including RING, one or two B-boxes and coiled-coil domains. In contrast, TRIMs C-terminal tails vary with different domains, based on which this protein family can be divided into 11 subfamilies (Figure 1-1) (31). Functionally speaking, thanks to the RING domains, TRIMs have been believed to be a large group of E3 ubiquitin (Ub) ligases. The B-box domains of TRIMs are regarded as zinc-binding motifs while coiled-coil domains play important roles in self-association and oligomerization of TRIM molecules, thereby acting as scaffolds to recruit and form multi-protein signaling complexes (28-31).

Roles of TRIMs in restriction of retroviruses are the first to be characterized, as exemplified by TRIM5 $\alpha$ which suppresses HIV-1 multiplication by catalyzing polyubiquitination of the viral capsid and blocking the viral uncoating step (28-31). A growing number of TRIMs have been progressively found to play direct antiviral roles in host restriction of viruses of other families by targeting important steps in the viral life cycle (Figure 1-2) (28-32). For example, TRIM22 polyubiquitinates IAV NP and targets it for proteasome-dependent degradation (33). In addition, TRIM56 was identified as a pleiotropic host antiviral factor restricting distinct RNA viruses via overlapping and distinct molecular determinants, including human coronavirus-OC43, IAV and Flaviviridae members (34-36).

Intriguingly, some TRIMs are also able to positively or negatively modulate the innate antiviral immune signaling pathways, thereby impacting IFN induction. On one hand, it is reported that approximately more than 30 TRIMs act at multiple levels of 


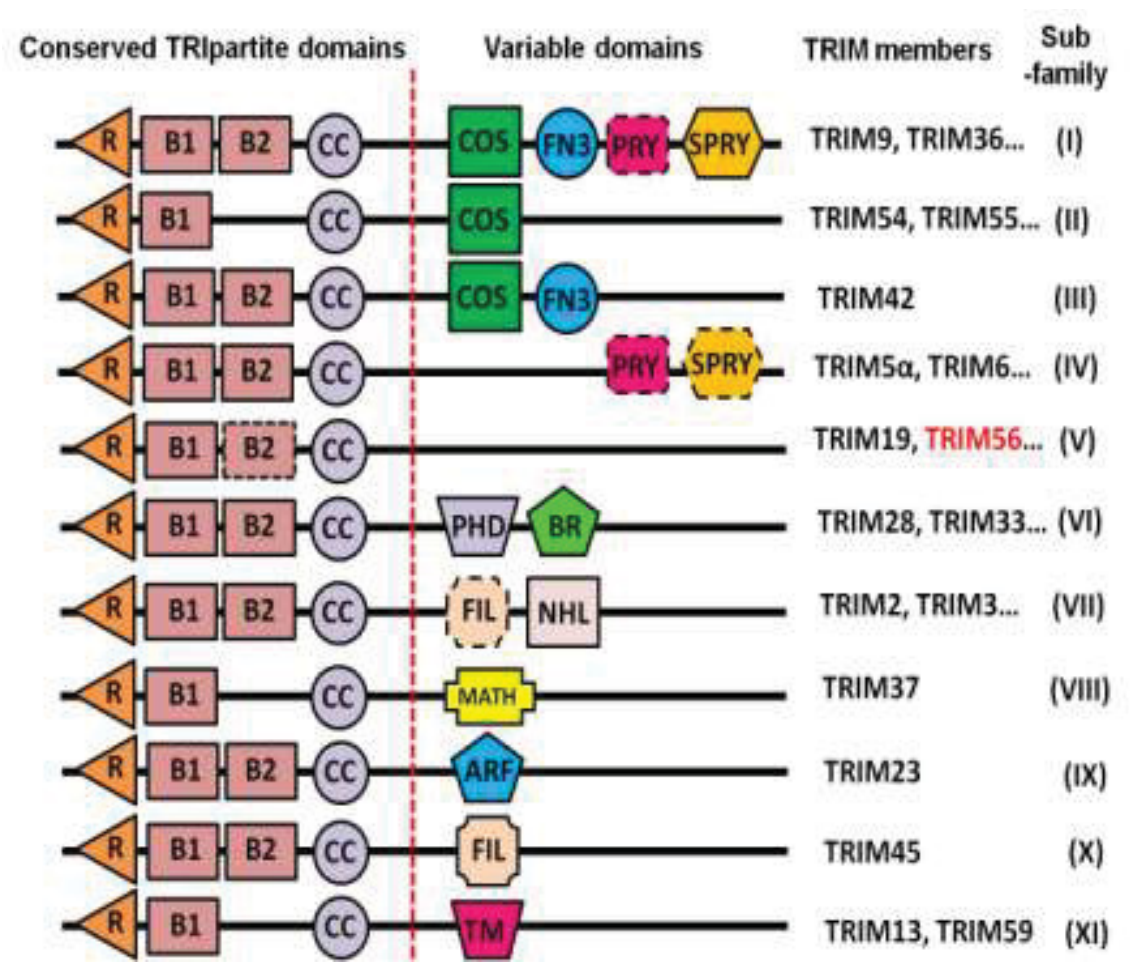

Figure 1-1. TRIM family members and their subfamily classification.

TRIM protein family members can be divided into up to 11 subfamilies based on the diversity of their C-terminal domain composition. 
Direct antiviral actions
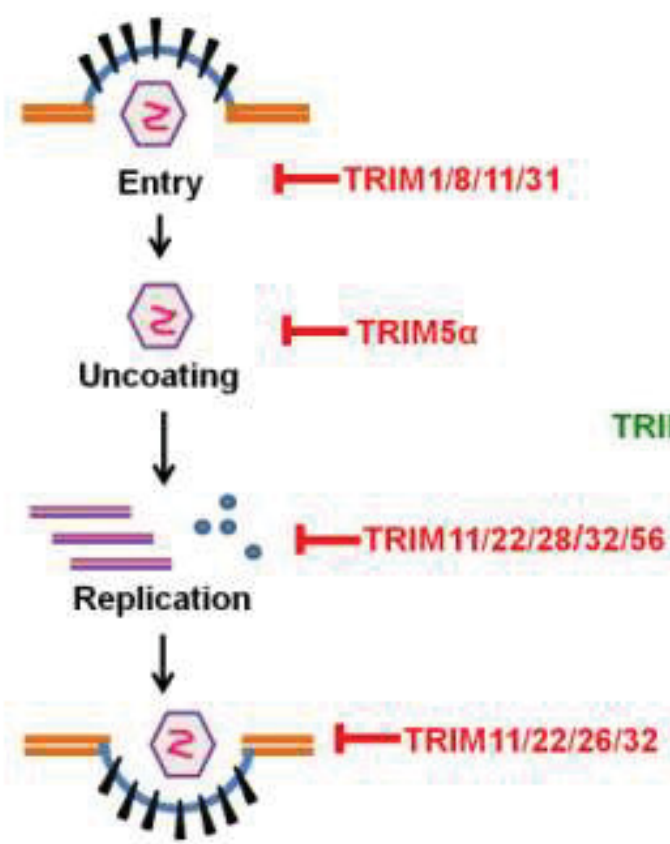

Packaging/release
Modulation of antiviral signaling

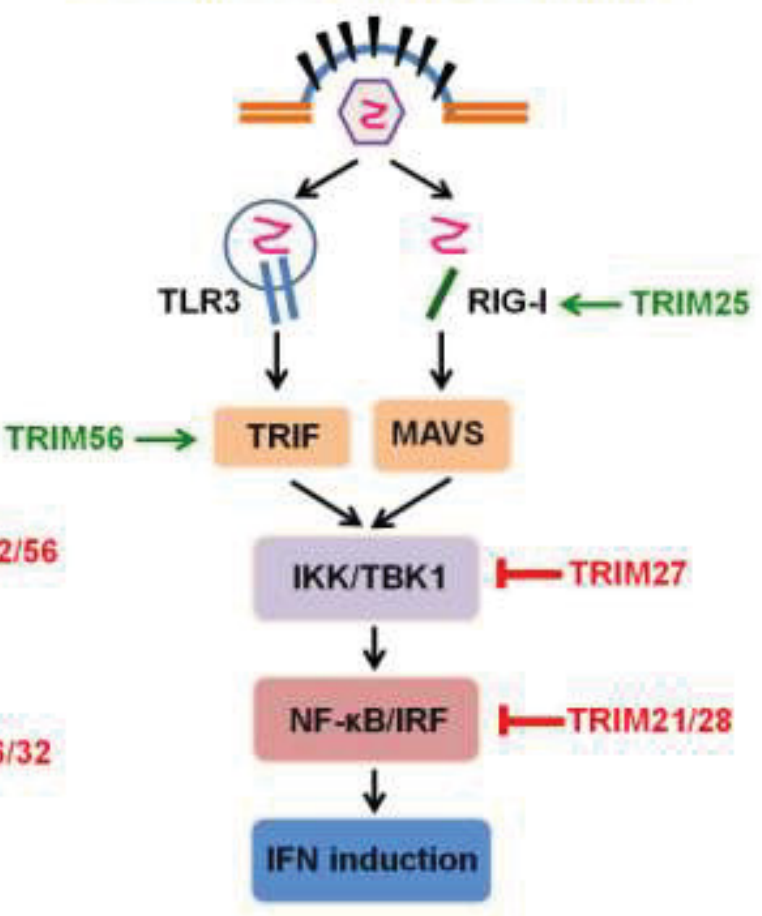

Figure 1-2. Roles of TRIMs in innate antiviral immunity.

(Left) TRIMs exhibit direct antiviral activity by targeting important steps of the viral life cycle. TRIM1/8/11/31 inhibit viral entry step. TRIM5 $\alpha$ prevents uncoating step of HIV-1. TRIM11/22/28/32 suppress retroviral gene transcription. TRIM56 restricts intracellular viral RNA replication of Flaviviridae members and influenza viruses. TRIM11/22/26/32 block virus packaging/release steps. (Right) TRIMs play important roles in modulation of PRR-mediated innate immune signaling pathways. TRIM25 ubiquitinates and activates RIG-I, thereby promoting IFN induction. TRIM56 potentiates TLR3 signaling by forming a complex with TRIF, the adaptor for TLR3. TRIM27 inhibits IKK activation. TRIM21/28 down-regulate IRF7 activation. 
innate immune signaling pathways to enhance antiviral responses (37). A prime example among this group of TRIMs is E3 ligase TRIM25 which substantially augments RIG-Imediated signaling pathway and IFN production by ubiquitinating and activating RIG-I (38). Another example is TRIM56, which has been demonstrated to significantly enhance TLR3-mediated signaling and establishment of an antiviral state by forming a complex with TRIF (39). On the other, a handful of TRIMs have been identified as negative regulators of PRR signaling, such as TRIM21 and TRIM27 down-regulating IKK activity and IRF7 activation, respectively $(29,30)$.

\section{Statement of Purpose}

The tripartite motif-containing (TRIM) proteins have emerged as a new class of host antiviral restriction factors, with several demonstrating roles in regulating innate antiviral responses. Of $>70$ known TRIMs, TRIM56 inhibits replication of bovine viral diarrhea virus, a ruminant pestivirus of the family Flaviviridae, but has no appreciable effect on VSV, a rhabdovirus. We have also shown that TRIM56 forms a complex with the Tolllike receptor-3 (TLR3) adaptor, TRIF, via its C-terminal residues 621-750, and augments TLR3-mediated interferon (IFN) induction and establishment of an antiviral state. Yet, TRIM56's antiviral spectrum and the precise underlying mechanisms by which TRIM56 executes its direct antiviral functions and modulates TLR3 signaling remain undefined. Also unclear are the molecular determinants governing the direct and indirect antiviral activities of TRIM56. The goal of this dissertation research has been to dissect the molecular determinants dictating TRIM56's novel antiviral activities against positiveand negative-strand RNA viruses and TRIM56-mediated positive regulation of the TLR3 antiviral signaling pathway as well as the molecular mechanisms by which TRIM56 executes its direct antiviral and signaling regulation functions.

- The objective of Chapter 3 is to dissect the molecular determinants dictating TRIM56's novel antiviral activities against medically important, positive-strand RNA viruses and the molecular mechanisms by which TRIM56 executes its direct antiviral functions against these viruses.

- The objective of Chapter 4 is to study the molecular determinants dictating TRIM56's novel antiviral activities against negative-strand RNA viruses and the molecular mechanisms by which TRIM56 exhibits its direct antiviral activities against these viruses.

- The objective of Chapter 5 is to investigate the molecular determinants dictating TRIM56-mediated positive regulation of the TLR3 antiviral signaling pathway and the molecular mechanisms by which TRIM56 executes its signaling regulation functions. 


\section{CHAPTER 2. METHODS AND MATERIALS ${ }^{1}$}

\section{Plasmids}

To construct C-terminally V5-tagged mouse TRIM56 (here referred to as mTRIM56 or mT56) expression vector (designated pEF6-mTRIM56-V5His), the cDNA encoding mTRIM56 was amplified by PCR using the primers 5'GAAGATCTATGAACTCCAAAGACTCCTCCCCAAC-3' (forward) and 5'GAATTCGCTGTCAGGAAACCTGACCCTAAAGA-3' (reverse), and subsequently ligated into $\mathrm{pEF} 6 / \mathrm{V} 5$-His TOPO (Invitrogen). The plasmid encoding N-terminal $2 \times \mathrm{HA}-$ tagged human TRIM56 in the pcDNA5/FRT/TO backbone (Invitrogen) has been described previously and designated as pcDNA5/FRT/TO-HA-TRIM56 (39). Plasmid vectors encoding various mutant (Mut) forms of TRIM56 were constructed from pcDNA5/FRT/TO-HA-TRIM56 by QuikChange site-directed mutagenesis (Stratagene). The vectors encoding C-terminally V5-tagged wild-type (WT) TRIM56 (designated pcDNA3.1-TRIM56-V5His) and its E3 ligase-null mutant (Mut) CC21/24AA and various deletion mutants were described elsewhere (34). S469A, S471A, S475A, S710A or S710D single mutation, or S469A+S471 A+S475A triple mutations were introduced into pcDNA3.1-TRIM56-V5His, and S689A into pEF6-mTRIM56-V5His by inverse PCR. The mutagenesis primers used are listed in Table 2-1. The gene fragments containing mutant TRIM56 (S469A, S471A, S475A, S710A, S710D or S469A+S471A+S475A) in pcDNA3.1 backbone were then swapped to pcDNA5/FRT/TO-HA backbone (Invitrogen) and the resultant vectors were designated pcDNA5/FRT/TO-HA-TRIM56 Mut. The coding sequences of the C-terminal-tail 135 and 63 residues (CTT135 and CTT63) of TRIM56 were amplified from a plasmid encoding full-length human TRIM56 via PCR and subsequently ligated into the pcDNA5/FRT/TO (Invitrogen) vector to generate the pcDNA5/FRT/TO-HA-TRIM56-CTT135 and -CTT63 constructs, respectively. In these constructs TRIM56-CTT135 and CTT63 were expressed as peptides fused in frame to an $\mathrm{N}$-terminal 2xHA epitope tag. A vector designated pCX4pur-FH-TRIM56 was constructed by swapping the cDNA fragment encoding TRIM56 from pcDNA5/FRT/TO backbone to pCX4pur-Flag-HA vector to ensure expression of TRIM56 fused with Nterminal Flag-HA tandem tags (FH-TRIM56). The identities of all plasmids were confirmed by DNA sequencing. pFlag-TRIF-mRHIM encoding a Flag-tagged,

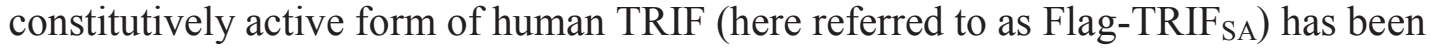
described elsewhere (39). pCMV1-Flag-TLR3 was a gift from Ruslan Medizhitov (Yale University).

${ }^{1}$ Adapted with permission. Liu B, Li NL, Wang J, Shi PY, Wang T, Miller MA, Li K. 2014. Overlapping and distinct molecular determinants dictating the antiviral activities of TRIM56 against flaviviruses and coronavirus. J Virol 88:13821-13835; Liu B, Li NL, Shen Y, Bao X, Fabrizio T, Elbahesh H, Webby RJ, Li K. The C-terminal Tail of TRIM56 Dictates Antiviral Restriction of Influenza A and B Viruses by Impeding Viral RNA Synthesis. J Virol 2016. pii: JVI.03172-15. 
Table 2-1. Mutagenesis primers for human (hT56) or mouse (mT56) TRIM56.

\begin{tabular}{ll}
\hline Mutations & Primer sequences $\left(5^{\prime} \rightarrow 3^{\prime}\right)$ \\
\hline hT56-S469A & Forward: ATATCCCGGGAGCCCAGCCCAG \\
& Reverse: CGCCTTGAGCCTGCCTTTGAAC \\
hT56-S471A & Forward: GCTCGAGAGCCCAGCCCAGCCCT \\
& Reverse: AATTGACTTGAGCCTGCCTTTGA \\
hT56-S475A & Forward: GCTCCAGCGCTGGGGCCGAATCT \\
& Reverse: GGGCTCCCGGGAAATTGACTTGA \\
hT56-S710A & Forward: GCTCTCCTTGGAGACTTCCTG \\
heverse: TCCCTTCGGGTCCAGGATCAC \\
\\
Forward: GATCTCCTTGGAGACTTCCTG \\
hT56- & Reverse: TCCCTTCGGGTCCAGGATCAC \\
S469A+S471A+S475A & Forward: \\
& Reverse: \\
mT56-S689A & TCGAGCAATAGCCTTGAGCCTGCCTTTGA \\
& Forward: GCTCTTCTTGGTGACTTCCTAAC \\
& Reverse: TCCCTTGGGATCCAGTATCAC \\
\hline
\end{tabular}


The plasmid pcDNA6-YFVpro contained the full-length NS2B-NS3 coding sequence of yellow fever virus (YFV)-17D in pcDNA6/V5-HisB backbone (40). Recombinant plasmids encoding the TSV01 strain of dengue virus-2 (DENV2) replicon (pACYC-TSVRep-WT) and its replication-deficient, NS4B P104R mutant (pACYC-TSV-NS4BP104R) were gifts from Pei-Yong Shi (41). The full-length $\mathrm{N}$ coding sequence of $\mathrm{HCoV}$ OC43 was amplified from cDNA of virally infected BSC-1 cells and ligated into $\mathrm{pEF} 6 / \mathrm{V} 5-\mathrm{His}-\mathrm{TOPO}$ (Invitrogen), to generate the pEF6-OC43-N-V5His construct in which the $\mathrm{N}$ gene is fused in frame to C-terminal V5-His6 epitope tags. The identities of all plasmids were confirmed by DNA sequencing.

Plasmids for luciferase (luc) reporter-based IAV RNA synthesis assay were gifts from Richard Webby (St. Jude Children's Research Hospital), which include polymerase IIdriven $\mathrm{pHW}$ plasmids expressing PB2, PB1, PA and NP of influenza A/PR/8/34 virus, and a polymerase I-driven luc reporter plasmid (pHY-Luci), which expresses in the negative sense the firefly luciferase flanked by noncoding sequences from the IAV matrix (M) segment (42).

\section{Cell Lines}

Human embryo kidney (HEK) 293, 293-TLR3 cells (HEK293 cells constitutively expressing human TLR3), HeLa, mosquito C6/36, African green monkey kidney Vero, Vero-E6, BSC-1 and Madin-Darby canine kidney (MDCK) epithelial cell lines were maintained in Dulbecco's Modified Eagle Medium supplemented with 10\% fetal bovine serum, $100 \mathrm{U} / \mathrm{ml}$ of penicillin and $100 \mu \mathrm{g} / \mathrm{ml}$ streptomycin. Madin-Darby bovine kidney (MDBK) and its derivative cells were grown in medium containing horse serum in lieu of FBS. Human hepatoma Huh7.5-TLR3 cells with TLR3 stable expression (Huh7.5-TLR3) were described previously (43). HEK293-T3Y-FH-T56 cells constitutively expressing FH-TRIM56 were generated by transducing HEK293-TLR3-YFP (293-T3Y, a gift from Kate Fitzgerald, University of Massachusetts) cells with replication-incompetent retroviruses carrying FH-T56 in pCX4pur backbone (pCX4pur-FH-TRIM56), followed by stable selection with puromycin. MDBK cell pools stably expressing C-terminal Flagtagged, WT or various mutant human TRIM56 (MDBK-T56 WT/Mut), or an empty vector (MDBK-Bsr), have been described elsewhere (34). Human osteosarcoma U2OS cell pools stably expressing TRIM56-Flag (referred to as U2OS-T56) and control U2OSBsr cells expressing an empty retroviral vector ( $\mathrm{pCX} 4-\mathrm{Bsr}$ ) were developed by retroviral gene transfer, followed by stable selection with blasticidin, as described (34).

HEK293 cells constitutively expressing WT or the E3 Ub ligase-deficient CC21/24AA mutant (Cys21 and Cys24 in the RING domain substituted with alanines) TRIM56 (designated as 293-T56 and 293-T56-CC21/24AA, respectively), were generated by transducing HEK293 cells with replication-incompetent retroviruses carrying C-terminal Flag-tagged WT and CC21/24AA mutant TRIM56 in pCX4bsr backbone, respectively, followed by stable selection with blasticidin (35). 
HeLa-Flp-In T-REx-ACE2 (FitA2) cells with tetracycline (tet)-inducible expression of HA-tagged, WT and various mutant versions of TRIM56, have been described and designated as HeLa-FitA2-T56 WT/Mut (39). Herein we created the HEK293 cell lines conditionally expressing WT or various mutant versions of TRIM56 (different deletion mutants, or CTT135, CTT63 or S469A, S471A, S475A, S710A or $\mathrm{S} 469 \mathrm{~A}+\mathrm{S} 471 \mathrm{~A}+\mathrm{S} 475 \mathrm{~A}$ ) using the Flp-In T-REx (FIT) expression system (Invitrogen) following the manufacturer's recommended protocol. In brief, 293-FIT cells were cotransfected with pOG44 encoding the Flp recombinase and pcDNA5/FRT/TO-HATRIM56 or the indicated TRIM56 mutants in pcDNA5/FRT/TO-HA backbone at 9:1 ratio, followed by stable selection of cells in media containing $200 \mu \mathrm{g} / \mathrm{ml}$ of hygromycin. The resultant cell lines were named 293-FIT-T56 or 293-FIT-T56-Mut, respectively. To induce the HA-TRIM56 (or mutant HA-TRIM56) expression in 293-FIT- and HeLaFitA2-derived cells, cells were cultured in tet-containing medium for $48 \mathrm{~h}$.

\section{Viruses, Viral Infections, Replication Assays and Miscellaneous Reagents}

YFV-17D (NR-115; BEI Resources) and DENV2 (Thailand 16681 strain) were propagated in Vero-E6 and C6/36 cells, respectively. Viral stock of HCoV-OC43 (ATCC VR-1558) was prepared in BSC-1 cells. EMCV (ATCC VR-1314, provided by Lawrence Pfeffer) was propagated in Vero cells. Viral stocks of influenza A/PR/8/34 (H1N1) virus (ATCC VR-1469) and B/Florida/4/06 (Yamagata-lineage) virus (provided by Richard Webby) were propagated and viral titers determined in MDCK cells. A recombinant vesicular stomatitis virus (VSV) expressing firefly luciferease, VSV-Luc, was a gift from Sean Whelan (Harvard Medical School). VSV-Luc stocks were prepared in Vero cells (44). Human metapneumovirus (hMPV) stocks were propagated and viral titers determined in LLC-MK2 cells (45). Sendai virus ( $\mathrm{SeV}$, Cantell strain) was purchased from Charles River Laboratories and used to infect cells at $100 \mathrm{HAU} / \mathrm{ml}$ for $12 \mathrm{~h}$. Viral infections of cells were conducted as described (34,40,46-48). Progeny infectious virus titers in cell-free culture supernatants were determined by endpoint dilution-based $50 \%$ tissue culture infective dose $\left(\mathrm{TCID}_{50}\right)$ assays in 96 -well plates $(43,49)$. Specifically, titration of YFV and EMCV was performed on Vero-E6 cells while HCoV-OC43 titration on BSC-1 cells. Cytopathic effect (CPE) was recorded and used for calculation of virus yield on 7 days post-infection (dpi) for YFV-17D and HCoV-OC43, and on 3 dpi for EMCV, respectively. Because infection by DENV2 does not cause obvious CPE, titration of virus yield was performed on Vero cells on 3 dpi using immunofluorescence staining to score prM-positive cell wells. TCID 50 assays was also performed to determine progeny infectious virus titers of IAV/IBV in cell-free culture supernatants, on monolayers of MDCK cells $(34,46,48)$. Cytopathic effect was recorded and used for calculation of infectivity at 24 hours postinfection (hpi) for IAV and $48 \mathrm{hpi}$ for IBV, respectively. All resultant viral titers were expressed as $\mathrm{TCID}_{50} / \mathrm{ml}$.

Poly-I:C (a dsRNA surrogate) was obtained from Sigma. To stimulate cells, poly-I:C (pIC) was added into culture medium at a final concentration of $20 \mu \mathrm{g} / \mathrm{ml}$. TNF- $\alpha$ and IL$1 \beta$ were used at a final concentration of $10 \mathrm{ng} / \mathrm{ml}$ for cell stimulation. 


\section{Transient Replicon Assay}

The Renilla luciferase-encoding, WT and NS4B P104R mutant DENV2 replicons were synthesized from Cla I-linearized plasmid templates by in vitro transcription as described elsewhere (41). 293-FIT-T56 cells cultured in the absence or presence of tet $\left(3 \times 10^{5}\right.$ cells) were transfected with $1 \mu \mathrm{g}$ of WT or the NS4B mutant replicon RNA using DMRIE-C (Invitrogen) as per instructions from the manufacturer. At $4 \mathrm{~h}$ post transfection, cells were split and seeded in 24-well plates. At various time points post transfection, cells were lysed in $200 \mu 11 \times 1$ ysis buffer (Promega). Thirty microliters of cell lysates were subject to Renilla luciferase assay (Promega).

\section{RNA Interference}

For stable knockdown of human TRIM56, we used a short hairpin RNA (shRNA) targeting the coding region of human TRIM56 gene inserted in the pLKO.1-puro vector that was purchased from Openbiosystems (referred to as shT56-094). The target sequence is: 5'-GCAGCAGAATAGTGTGGTAAT-3' (34-36). In addition, for stable knockdown of endogenous human TRIM56 without impact on the expression of exogenously introduced TRIM56, I used another shRNA which specifically targets the 3' untranslated region (UTR) region of TRIM56 gene and was inserted in the pLKO.1-puro vector (referred to as shT56 \#093). The target sequence is: 5'CGTCTTCTAGTGTGTGAGAAT-3'. As a negative control, a non-targeting scrambled shRNA, also cloned in pLKO.1-puro (Addgene \#1864), was used (34-36). The shRNAs were packaged into replication-incompetent lentiviral particles in 293FT cells, and subsequently used to infect indicated cell lines. Following selection in medium containing 2-5 $\mu \mathrm{g} / \mathrm{ml}$ of puromycin for 2-3 weeks, surviving cell colonies were pooled for further analysis. For transient knockdown of endogenous TRIM56 in 293-FIT-T56WT/Mut cells without down-regulating the expression of exogenously introduced HATRIM56-WT/Mut, I employed an siRNA (Fisher Scientific) that specifically targets the 3' UTR of human TRIM56 transcript (35). This siRNA had a target sequence of 5'GCCGCTGCTATATAGTTTA-3', and was transfected into cells at a final concentration of $100 \mathrm{nM}$ by Lipofectamine 2000 (Invitrogen) as per the manufacturer's instructions. To silence the expression of STING, I transfected cells with a mixture of two shRNAs targeting the coding region of human STING transcript (TRCN0000160281 and TRCN0000163296, Openbiosystems). To knockdown TRIF or RIG-I, I used synthetic siRNAs that have previously been described $(11,50)$. The target sequences were: TRIF1077, GAAGATACCACCTCTCCAA; RIG-I, GGAAGAGGTGCAGTATATT.

MDBK cells with stable knockdown of endogenous bovine TRIM56 (boT56) have been described elsewhere (34). To knock down exogenously expressed human TRIM56 in MDBK-T56 cells, shT56-\#094 or a non-targeting scrambled shRNA (as a negative control) were packaged into replication-incompetent lentiviral particles in HEK293FT cells, and subsequently used to transduce MDBK-T56 cells, respectively (36). Following 
selection in medium containing 2-5 $\mu \mathrm{g} / \mathrm{ml}$ of puromycin for 2-3 weeks, survived cell colonies were pooled for further analysis.

\section{RNA Analyses}

Extraction of total cellular RNA by TRIzol, cDNA synthesis by reverse transcription and quantitative PCR (qPCR) were implemented as described elsewhere $(34,51)$. The following primers were used: ISG56 and 28S (39); IFN- $\beta$ (51); IL29, 5'GAAGAGTCACTCAAGCTGAAAAACTG-3' (forward) and 5'GAGAAGCCTCAGGTCCCAATT-3' (reverse); TRIM56 coding region (34); TRIM56 3'UTR, 5'-CCGAGGACATTTTCCTGAAG-3' (forward) and 5'AGTTAAGGTCACGCCACCAC-3' (reverse); TRIF, 5' GTGGAGGAAGGAACAGGACA-3' (forward) and 5'TGAGTAGGCTGCGTTCAGTG-3' (reverse); RIG-I, 5'GGACGTGGCAAAACAAATCAG-3' (forward) and 5'ATTGTGATCTCCACTGGCTTTGA-3' (reverse); STING, 5' TCAAGGATCGGGTTTACAGC-3' (forward) and 5'TGGCAAACAAAGTCTGCAAG-3' (reverse); YFV NS2B region, 5' TGAACAAGGGGAGTTCAAGC-3' (forward) and 5'AGGACCAGCAGAAGAGCAAA-3' (reverse); HCoV-OC43 N gene, 5'CGATGAGGCTATTCCGACTAGGT-3' (forward) and 5'CCTTCCTGAGCCTTCAATATAGTAACC-3'(reverse) (52). The relative abundance of each target was normalized to that of $28 \mathrm{~S}$ rRNA. Copy numbers of YFV and HCoVOC43 RNAs were calculated based on standard curves generated using serially diluted pcDNA6-YFVpro and pEF6-OC43-N-V5His DNA, respectively.

To analyze the abundance of three different influenza viral RNA species, RT was performed using strand- and sense-specific oligonucleotides for vRNA, anti-genomic complementary RNA (cRNA) and mRNA as previously described (26). A 28S-specific primer (5' CTTAACGGTTTCACGCCCTC 3') was also included in the RT reaction mixture for vRNA or cRNA analysis. Subsequently the following primers were used for qPCR: $M$ segment of influenza A/PR/8/34 virus, 5' GACCRATCCTGTCACCTCTGAC 3' (forward) and 5' AGGGCATTYTGGACAAAKCGTCTA 3' (reverse); and hemaglutinin (HA) segment of influenza A/PR/8/34 virus, 5' GGCCCAACCACAACACAAAC 3' (forward) and 5' AGCCCTCCTTCTCCGTCAGC 3' (reverse). The qPCR primers for measuring mRNA abundance of $\mathrm{SeV} P$ gene are, 5'CTCTGGGAGAACAAGCAAGC-3' (forward) and 5'-

TCGCCCAGATCCTGAGATAC-3' (reverse). The relative abundance of each target was normalized to that of $28 \mathrm{~S}$ rRNA. 


\section{Luciferase Reporter-based Minigenome Replication Assay of IAV RNA Synthesis}

Luciferase reporter-based minigenome replication assay for monitoring IAV transcription and replication was performed as previously described $(42,53,54)$. In brief, HEK293-FIT-T56-WT or -Mut cells cultured in the presence or absence of tet (to induce or repress HA-T56 WT/Mut expression, respectively) were co-transfected using Lipofectamine 3000 (Invitrogen) with polymerase II-driven pHW plasmids expressing RNP complex genes (PB2, PB1, PA and NP) of influenza A/PR/8/34 virus, and a polymerase I-driven luciferase reporter plasmid pHY-Luci (42). Also co-transfected was a CMV promoter-driven plasmid expressing the Renilla luciferase (pRL-CMV, Promega), which served as an internal control for normalization of transfection efficiency. At the indicated time points post transfection, cells were lysed and processed for dual-luciferase assay.

\section{Immunoblotting, Immunofluorescence Staining, and Confocal Microscopy}

Cell lysates were prepared in RIPA buffer and subjected to SDS-PAGE and immunoblot analysis as previously described $(34,50)$. Immunofluorescence staining and confocal microscopy were performed as previously described $(34,50)$. The following monoclonal $(\mathrm{mAb})$ and polyclonal antibodies $(\mathrm{pAb})$ were utilized: rabbit anti-YFV NS3 $\mathrm{pAb}$ (a gift from Charles Rice), which we found to cross-react with DENV2 NS3; mouse anti-DENV mAb D1-11(3) (Genetex); mouse anti-DENV prM (clone 2H2) hybridoma culture supernatant; goat anti-DENV (BEI Resources), which we found to recognize predominantly prM; anti-EMCV 3D pol mAb (a gift from Ann Palmenberg); mouse antiHCoV-OC43 N mAb (Millipore); mouse anti-influenza A/WSN/33 (H1N1) NP 5/1 mAb (confirmed to react with the NP of $\mathrm{A} / \mathrm{PR} / 8 / 34$ virus) and goat anti matrix (M) protein antiserum (gifts from Richard Webby); goat anti-influenza B/Hong Kong/8/73 HA pAb (BEI Resources NR-3165), which also reacts with the HA of B/Florida/4/06 virus; rabbit anti-SeV pAb (a gift from Ilkka Julkumen); rabbit anti-hMPV pAb (45); rabbit antiTRIM56 pAb; rabbit anti-TRIF pAb; mouse anti-HA tag mAb (Invivogen), which I have confirmed not to react with the HA of either $\mathrm{A} / \mathrm{PR} / 8 / 34$ or B/Florida/4/06 virus; mouse anti-HA tag $\mathrm{mAb}$ (clone 12CA5, Roche); mouse anti-V5 tag mAb (Invivogen); mouse anti-Flag M2 mAb (Sigma); mouse anti-actin mAb (Sigma); rabbit anti- $\beta$ tubulin pAb (Santa Cruz); peroxidase-conjugated secondary goat anti-rabbit, goat anti-mouse and rabbit anti-goat pAbs; FITC-conjugated secondary goat anti-mouse pAb (Southern Biotech); and Alexa Fluor 594-conjugated secondary donkey anti-mouse and chicken anti-goat pAbs (Invitrogen). Densitometry quantification of protein bands of interest was performed using the Image J software (NIH). 


\section{Reporter Gene Assay}

IFN- $\beta$, IL-29 and PRDII promoter activities in transfected cells after different treatments were measured using a luc reporter assay as described $(39,50)$.

\section{Statistical Analysis}

Statistical differences were analyzed using students' $t$-test with the SPSS 11.5 software where appropriate. All $P$ values were two-tailed and $P<0.05$ was regarded to be statistically significant. Error bars represent standard deviations. 


\section{CHAPTER 3. OVERLAPPING AND DISTINCT MOLECULAR DETERMINANTS DICTATING THE ANTIVIRAL ACTIVITIES OF TRIM56 AGAINST FLAVIVIRUSES AND CORONAVIRUS ${ }^{1}$}

\section{Introduction}

The innate immune system represents a primary and fundamental component of host defense in the immediate-early phase of microbial infections. It is equipped with multilayered mechanisms. As sentinels to alert cells to the presence of invading viral pathogens, pattern recognition receptors (PRRs) such as the toll-like receptors (TLRs) and retinoic-inducible gene-I (RIG-I)-like receptors sense viral nucleic acids, a major class of viral pathogen-associated molecular pattern, activating intracellular signaling pathways that culminate in production of interferons (IFNs). These antiviral cytokines act in a paracrine/autocrine fashion to induce the expression of hundreds of interferonstimulated genes (ISGs) that collectively establish an antiviral state, reining in viral replication and spread (1-4). In addition to the IFN system, mammalian hosts encode a number of intracellular restriction factors that have direct antiviral roles (5). Although most of these factors are constitutively expressed at variable levels, many are, not surprisingly, also shown to be IFN-inducible. Of these, members of the tripartite motifcontaining (TRIM) protein family are increasingly recognized as active players in antiviral innate immunity (28-30).

The TRIM protein family comprises more than 70 members whose functions span a broad array of physiological processes, including cell proliferation, differentiation, and development, etc $(28,31)$. Structurally, TRIM family members have highly conserved arrangement of N-terminal tripartite motifs, i.e., RING, one or two B-boxes, and coiledcoil domains, and variable C-terminal region that has or has not up to four known functional domains (for example, the PRY-SPRY motifs). In general, RING domains confer TRIM proteins with E3 ubiquitin (Ub) ligase activity and the ability to recognize E2 conjugating enzyme. B-box domains are considered as zinc-binding motifs important for protein-protein interactions while coiled-coil domains facilitate self-association, turning oligomerized TRIM family proteins into a scaffold for assembling multi-protein complexes. In contrast, the C-terminal half of the TRIM family proteins have been proposed to be involved in protein-protein interactions, for example, presenting the substrate to an E2 conjugating enzyme. Based on the variability in C-terminal domain composition, TRIMs can be classified into up to 11 subfamilies (C-I to C-XI), with nine TRIM family members that lack a known C-terminal domain setting up subfamily $\mathrm{C}-\mathrm{V}$ $(30,31)$.

\footnotetext{
${ }^{1}$ Adapted with permission. Liu B, Li NL, Wang J, Shi PY, Wang T, Miller MA, Li K. 2014. Overlapping and distinct molecular determinants dictating the antiviral activities of TRIM56 against flaviviruses and coronavirus. J Virol 88:13821-13835.
} 
As exemplified by TRIM5 $\alpha$, the first and most characterized role in antiviral immunity of TRIMs is their ability to act as retroviral restriction host factors $(28,29,32)$. In recent years, however, a small number of TRIMs have been appreciated for novel roles in modulating antiviral innate immune signaling and in possessing direct antiviral activities against viruses of other families (29-31). Of a handful of TRIMs falling into the latter group, TRIM79 $\alpha$, a rodent-specific TRIM, restricts tick-borne encephalitis virus (TBEV), a classical flavivirus, by targeting the nonstructural (NS)-5 protein for lysosome-dependent degradation (55). TRIM56, a C-V subfamily member, is a virus- and IFN-inducible factor possessing antiviral activity against bovine viral diarrhea virus (BVDV) (34), a ruminant pestivirus classified within the family Flaviviridae shared by TBEV. Although the precise mechanism of action remains elusive, the antiviral effect is abolished by mutations that abrogate the E3 Ub ligase activity or by deletions within the last $\sim 130$ amino acids (aa) of the C-terminus, suggesting the importance of the RING domain-residing E3 ligase activity and C-terminal integrity in TRIM56's anti-BVDV actions. However, it is unknown whether the B-box or coiled-coil domains or other parts of the C-terminal region also play a part in the antiviral barrier function of TRIM56. Also left unanswered is the question of the specificity and spectrum of TRIM56's antiviral actions. Of particular interest, whether TRIM56 impacts medically important RNA viruses has not been determined.

This study is the first to demonstrate that human TRIM56 possesses antiviral activity against yellow fever virus (YFV) and dengue virus (DENV), two classical flaviviruses that are threatening the well-beings of approximately half of the world's population (56). Additionally, I report that TRIM56 restricts a human coronavirus, $\mathrm{HCoV-OC43,} \mathrm{which} \mathrm{is}$ responsible for a significant share of common cold cases. In contrast, I find that TRIM56 does not impact propagation of encephalomyocarditis virus (EMCV), a picornavirus, suggesting TRIM56 does not act on positive-strand RNA viruses indiscriminately. By extensive domain mapping, I discovered that distinct molecular determinants underpin the observed antiviral effects against different viral families. Consistent with this, I found that while TRIM56 inhibits flavivirus RNA replication, it acts at the stage of coronavirus packaging/release. These data delineate the antiviral spectrum of TRIM56 against

positive-strand RNA viruses and shed novel light on the molecular basis of the versatility and specificity, and the mechanisms of action of this host restriction factor against medically-important RNA viruses.

\section{Results}

\section{Ectopic Expression of TRIM56 Inhibits Propagation of YFV, DENV2 and HCoV- OC43, but Not That of EMCV}

We have previously reported that both human and bovine TRIM56 restrict the replication of BVDV, a ruminant pestivirus in the family Flaviviridae, but have no demonstrable effect on vesicular stomatitis virus (VSV), a rhabdovirus (34). To understand the specificity and spectrum of the antiviral activity of TRIM56 against 
positive-strand RNA viruses, I determined the impact of human TRIM56 overexpression on multiplication of four RNA viruses of three distinct viral families. These included two medically-important flaviviruses, YFV and DENV2; a human coronavirus, HCoV-OC43; and a picornavirus, EMCV.

To gauge the effect of TRIM56 without the confounding factor of clonal variation, I established HEK293 cells with conditional expression of N-terminal HA-tagged TRIM56 using the FIT expression system. This strategy was chosen since it enables the development of isogenic, stable cell lines harboring tet-inducible expression of a protein of interest from a specific genomic location. The resultant cell line, designated as 293FIT-T56, exhibited no detectable HA-TRIM56 protein when cultured in the absence of tet (-tet) but demonstrated robust HA-TRIM56 expression upon tet addition to culture medium (+tet) (Figure 3-1A, left panel, compare lane 1 vs 2). When infected with YFV$17 \mathrm{D}$ at an MOI of 0.1 , cells without HA-TRIM56 expression (-tet) harbored high levels of viral NS3 antigen at 24 hours post-infection (hpi) (lane 3). In contrast, in cells induced for HA-TRIM56 expression (+tet) NS3 expression was abrogated (lane 4). In agreement with this, there was an 8 -fold reduction $(P<0.05)$ in progeny virus titers in supernatants of 293-FIT-T56 cells cultured in the presence of tet as compared with those in the absence of tet (Figure 3-1A, right panel). Similar results were obtained in HeLa cells with tet-inducible expression of HA-TRIM56, i.e., HeLa-FitA2-T56 (Figure 3-1B), confirming that the finding is not a cell type-specific phenomenon. We also observed that stable, constitutive expression of C-terminal Flag-tagged TRIM56 (TRIM56-Flag) in HEK293 cells via retroviral gene transfer (i.e., 293-T56 cells), blunted YFV-17D NS3 protein expression and progeny virus production over a 3-day observation period as compared to control HEK293 cells (data not shown). Thus, the anti-YFV activity was also reproduced when ectopic expression of TRIM56 was achieved using a different expression system.

I next examined how TRIM56 overexpression affected propagation of DENV2, a flavivirus closely related to YFV. As in the case of YFV-17D, replication of the 16681 strain of DENV2 was crippled in 293-FIT-T56 cells upon turning on HA-TRIM56 expression, as revealed by diminished expression of viral E protein (Figure 3-1C, left panel, lane 3 vs 4$)$ and an approximately 1-log decrease $(P<0.05)$ in infectious virus production (right panel). Moreover, substantial reduction in prM protein abundance was observed in HeLa-FitA2-T56 cells infected with DENV2 when HA-TRIM56 expression was induced by addition of tet (Figure 3-1D, compare lanes 4 vs 3), corroborating TRIM56-mediated inhibition of DENV2 infection in another cell line.

Since TRIM56 is most abundantly expressed in the lungs (34), I reasoned that TRIM56 might contribute to cellular defense against respiratory viruses. I thus investigated whether manipulation of TRIM56 abundance alters cellular permissiveness for $\mathrm{HCoV}-\mathrm{OC} 43$, a human coronavirus that typically causes common cold. Indeed, an antiviral effect of TRIM56 was observed in 293-FIT-T56 cells infected with HCoVOC43, with cells induced for HA-TRIM56 expression exhibiting substantially lower viral $\mathrm{N}$ protein abundance and producing $1-\log (P<0.05)$ fewer infectious viruses in culture medium than cells not induced (Figure 3-1E). 

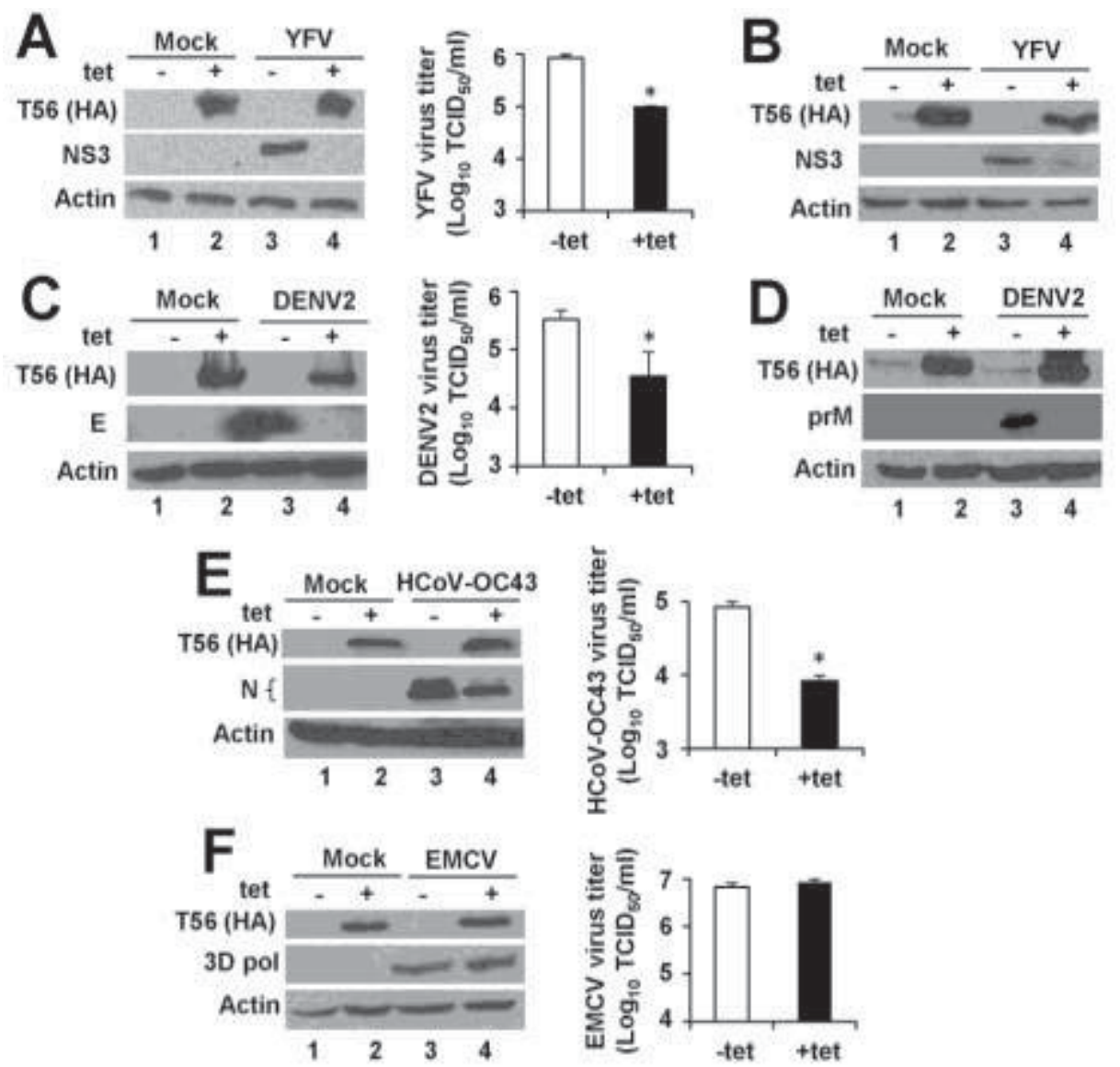

Figure 3-1. Ectopic expression of TRIM56 inhibits multiplication of YFV, DENV2 and $\mathrm{HCoV}-\mathrm{OC} 43$, but not that of EMCV.

(A) Left: Immunoblot analysis of the expression of N-terminal HA-tagged TRIM56 (using anti-HA antibody) and YFV NS3 in 293-FIT-T56 with or without tetracycline (tet) and mock infected or infected with YFV-17D for $24 \mathrm{~h}(\mathrm{MOI}=0.1)$. Right: Progeny virus production was determined by TCID $_{50}$ assay in culture supernatants of 293-FIT-T56 cells with or without tet treatment at 24 hours post-infection (hpi) with YFV-17D (MOI = 0.1). Asterisk $\left(^{*}\right)$ denotes that statistical differences exist between -tet and +tet cells with a $P$ value of $<0.05$. (B) HeLa-FitA2-T56 cells inhibited NS3 protein expression at 72 hpi of YFV-17D $(\mathrm{MOI}=0.5)$. (C, E and F) Left panels: Immunoblot analysis of of HATRIM56 and viral protein expression in mock- or viruses-infected 293-FIT-T56 cells with or without tet treatment. Right panels: progeny viral titers for the infected cells in the absence or presence of tet treatment, under similar experimental settings of (A) except that the cells were infected with DENV2-16681 strain $(\mathrm{MOI}=0.1)$ for $24 \mathrm{~h}$ for immunoblot analysis and $72 \mathrm{~h}$ for $\mathrm{TCID}_{50}$ assay in $(\mathrm{C})$, with $\mathrm{HCoV}-\mathrm{OC} 43(\mathrm{MOI}=0.1)$ for $24 \mathrm{~h}$ in $(\mathrm{E})$, or with EMCV $(\mathrm{MOI}=0.01)$ for $18 \mathrm{~h}$ in $(\mathrm{F})$, respectively. The viral protein antibodies used for immunoblotting included anti-DENV2 envelope (E) in (C), anti-HCoV-OC43 nucleocapsid (N) protein in (E), and anti-EMCV 3D polymerase (pol) in (F). (D) Tet-inducible expression of HA-TRIM56 in HeLa-FitA2-T56 cells inhibited prM protein expression at $36 \mathrm{hpi}$ of DENV2 $(\mathrm{MOI}=0.1)$. 
Given that all positive-strand RNA viruses replicate on cytoplasmic membranes (57) and that TRIM56 exhibits a cytoplasmic distribution, I wondered whether TRIM56 also inhibits other positive-strand RNA viruses. I thus examined the effect of conditional expression of HA-TRIM56 on propagation of EMCV, a prototype member of the family Picornaviridae. Western blotting revealed 293-FIT-T56 cells with or without HATRIM56 induction supported comparable production of 3D polymerase protein, the replicase core of EMCV, following infection with the virus $(\mathrm{MOI}=0.01)($ Figure 3-1F, left panel). In line with this, there was no appreciable difference in progeny virus yields in culture supernatants between tet-treated and tet-untreated cells (Figure 3-1F, right panel). These data reveal that although ectopic expression of TRIM56 impedes the multiplication of flaviviruses (YFV and DENV2) and HCoV-OC43, it does not target positive-strand RNA viruses indiscriminately, since TRIM56 has no demonstrable antiviral activity against EMCV.

In aggregate, the aforementioned experiments delineate the antiviral spectrum of TRIM56 against positive-strand RNA viruses and reveal that when ectopically expressed, this TRIM protein acts to curb infections by flaviviruses (YFV and DENV2) and HCoVOC43.

\section{TRIM56 Expressed at Physiologic Levels Restricts Infections by YFV, DENV2 and HCoV-OC43}

Next, I determined whether TRIM56 expressed at physiologic levels poses a barrier to infection with the three RNA viruses identified above. To achieve this goal, I stably knocked down endogenous TRIM56 and measured the impact on viral protein expression and progeny virus production. Compared with that in HeLa cells bearing a non-targeting, scrambled control (Ctrl) shRNA, TRIM56 protein abundance in pools of HeLa cells stably transduced with a TRIM56 shRNA (HeLa-shT56-094 cells) was reduced by $\sim 80 \%$ (Figure 3-2A, left panel). When infected with YFV-17D, HeLa-shT56-094 cells had markedly elevated NS3 protein levels and a $>1-\log$ increase $(P<0.05)$ in progeny virus yields as compared with HeLa-Ctrl cells (Figure 3-2A). Moreover, the enhanced YFV replication in TRIM56 knockdown cells was confirmed by immunostaining of YFV NS3 expression (data not shown). TRIM56 depletion also rendered cells more permissive to DENV2. As shown in Figure 3-2B, following DENV2 infection, we observed elevated DENV NS3 expression (immunoblotting data in left panel), increased progeny virus yields (middle panel) and higher percentage of cells with discernable prM expression (right panel) in TRIM56 knockdown cells compared with the control cells. Thus, the effects of TRIM56 depletion on DENV mirrored with those seen in YFV-17D-infected cells. The same can be said concerning the impact of TRIM56 silencing on HCoV-OC43 infection; we found that HeLa-shT56-094 cells supported higher levels of viral N protein expression and produced 6 -fold more $(P<0.01)$ progeny viruses than HeLa-Ctrl cells (Figure 3-2C). Collectively, the TRIM56 knockdown experiments corroborate the physiologic relevance of the finding that TRIM56 is an intrinsic host restriction factor of YFV, DENV2 and HCoV-OC43. 

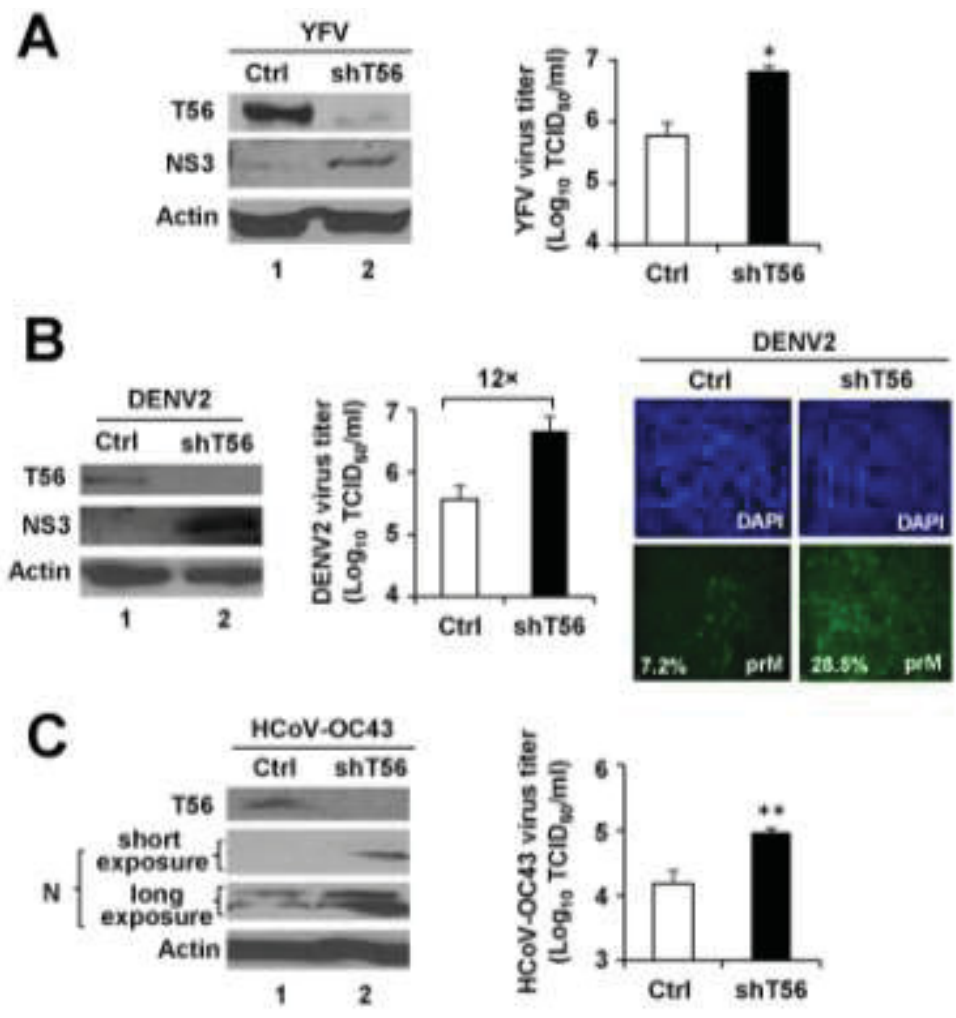

Figure 3-2. The endogenous TRIM56 protein restricts propagation of YFV, DENV2 and HCoV-OC43.

(A) Left panel: Immunoblot analysis of the expression of TRIM56 (using anti-TRIM56 antibody) and YFV NS3 (using anti-YFV NS3 antibody) in HeLa cells stably transduced with either a non-targeting, scrambled control shRNA (Ctrl) or TRIM56 shRNA-094 (shT56) at 72 hpi with YFV-17D $(\mathrm{MOI}=0.5)$. Actin served as a loading control to demonstrate equal sample loading. Right panel: Culture supernatants of virus-infected cells were harvested at $36 \mathrm{hpi}$ and subjected to $\mathrm{TCID}_{50}$ assay for determination of progeny virus production. (B) Left panel: Immunoblot analysis of TRIM56 and DENV2 NS3 (using anti-YFV NS3) expression in HeLa-Ctrl and HeLa-shT56-094 cells at 60 hpi with DENV2-16681 (MOI = 1.5). Middle panel: Progeny virus titers in culture supernatants of the DENV2-infected cells (MOI = 0.5, $60 \mathrm{hpi})$. Note that HeLa-Ctrl cells produced 12-fold fewer progeny viruses than HeLa-shT56-094 cells. Right panel: Immunostaining of DENV2 prM expression (using DENV 2H2 hybridoma supernatant) in HeLa-Ctrl and HeLa-shT56-094 cells at 60 hpi with DENV2-16681 strain (MOI= $0.5)$. The mean percentage of cells with discernable prM expression was quantified from three independent images and shown at the lower left corner of each prM panel. (C) Left panel: Immunoblot analysis of TRIM56 and N protein (N) (using anti-HcoV OC43 N) expression in HeLa-Ctrl and HeLa-shT56-094 cells at 48 hpi with HCoV-OC43 (MOI = 0.01). Right panel: Progeny virus titers in culture supernatants of the virus-infected cells. “*” and "**" indicate that statistical differences exist between Ctrl and shT56 cells with a $P$ value of $<0.05$ and $<0.01$, respectively. 


\section{The Antiviral Effects of TRIM56 Are Not Attributed to General Augmentation of IFN Response or to Regulation of Cell Growth}

TRIM56 possesses the ability to promote type I IFN response induced via the TLR3 signaling pathway (39). In light of this, I studied whether heightened induction of the IFN antiviral response is responsible for the retarded viral propagation in TRIM56-expressing cells. To this end, I examined induction of ISG15 and/or ISG56, well-characterized sensitive markers of the IFN response, in cells with or without TRIM56 expression and infected with YFV and HCoV-OC43, respectively. As shown in Figure 3-3A, there was no detectable ISG15 protein in control HEK293 or 293-T56 cells infected with YFV17D, although robust induction of ISG15 was observed in these cells following Sendai virus infection. Thus, regardless of the status of TRIM56 expression, there is no discernable IFN response when infected with YFV. Likewise, expression of ISG15 or ISG56 was undetectable in 293-FIT-T56 with or without HA-TRIM56 induction, and prior to and after $\mathrm{HCoV}-\mathrm{OC} 43$ infection (Figure 3-3B), despite that there was a substantial decline in N protein abundance in these cells upon turning on HA-TRIM56 expression (lanes 4 vs 3, Figure 3-3B). Consistent with these data, there was no significant up-regulation of transcription for ISG56, IFN- $\beta$, or IL-29, in 293-FIT-T56 cells with (+tet) or without (-tet) HA-TRIM56 induction when infected with YFV, DENV2 or HCoV-OC43 (data not shown). I conclude from these data that general augmentation of the IFN antiviral response is not responsible for the antiviral effects of TRIM56 against flaviviruses and $\mathrm{HCoV}-\mathrm{OC} 43$.

I also considered the possibility that TRIM56 may impact cell growth, producing a condition unfavorable for viral propagation. However, I did not find any difference in cell proliferation between 293-FIT-T56 cells induced and repressed for HA-TRIM56 expression over a seven-day observation period (Figure 3-3C), nor did I observe obvious cell death or apoptosis before and following tet-induction of HA-TRIM56 (data not shown).

\section{TRIM56's Antiviral Actions Do Not Depend upon TLR3/TRIF, RIG-I or STING}

TRIM56 has been reported to form a complex with TRIF and STING, thereby potentiating TLR3 and STING-dependent antiviral signaling pathways, respectively $(39,58)$. Although I did not find heightened IFN or ISG induction to be responsible for TRIM56's antiviral activities, the possibility could not be ruled out that IFN-independent mechanisms through the TLR3/TRIF-, RIG-I-, or STING-axis may contribute. To clarify this, I determined whether knockdown of TRIF, RIG-I or STING alters the antiviral activities of TRIM56 against YFV and HCoV-OC43. HEK293-derived cells generally have very low expression of TLR3, and I found this to be the case with the 293-FIT cells (data not shown). In line with this, there was little induction of ISG56 transcript in extracellular poly-I:C-stimulated cells without HA-TRIM56 induction (Figure 3-4A, tet). Turning on HA-TRIM56 expression (+tet) strongly augmented induction of ISG56 by poly-I:C, but this response was curtailed upon TRIF silencing (Figure 3-4A). This is consistent with our previous report that TRIM56 positively regulates TLR3 


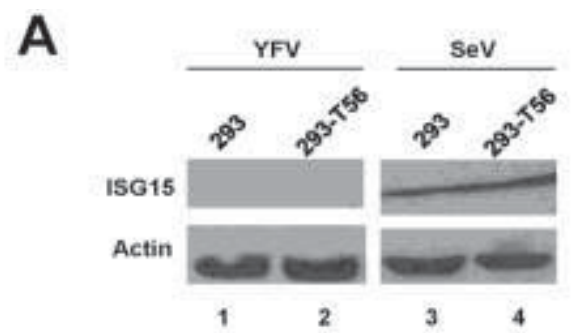

B
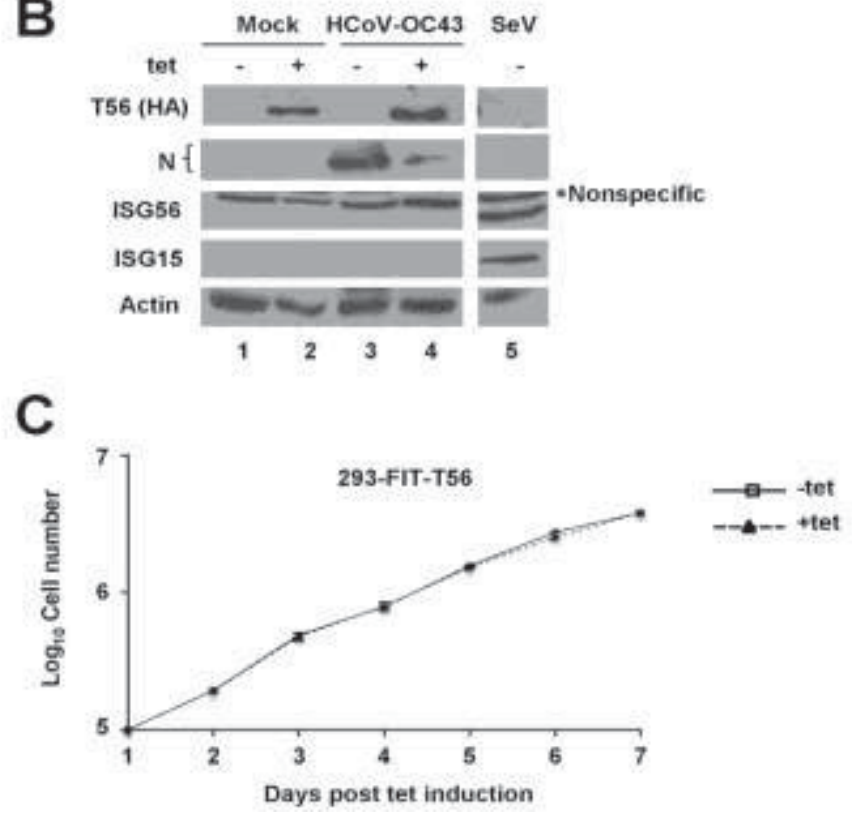

Figure 3-3. The antiviral effects of TRIM56 do not result from up-regulation of IFN response or from modulation of cell growth.

(A) Immunoblot analysis of ISG15 protein abundance in 293 cells or cells stably overexpressing TRIM56-Flag (293-T56) at $24 \mathrm{hpi}$ with YFV-17D $(\mathrm{MOI}=0.1)$ or at 12 hpi with Sendai virus $(\mathrm{SeV})$ as a positive control for induction of the IFN response. (B) Immunoblot analysis of HA-TRIM56, HCoV-OC43 N protein, ISG15, and ISG56 expression in 293-FIT-T56 cells that were mock infected or infected with HCoV-OC43 $(\mathrm{MOI}=0.1)$ for $24 \mathrm{~h}$ in the absence (odd numbered lanes) or presence (even numbered lanes) of tet treatment. Asterisk indicates a nonspecific band detected by the anti-ISG56 antibody. (C) Growth curves of 293-FIT-T56 cells cultured with or without tet was assessed by cell number counts over a 7 -day observation period. 

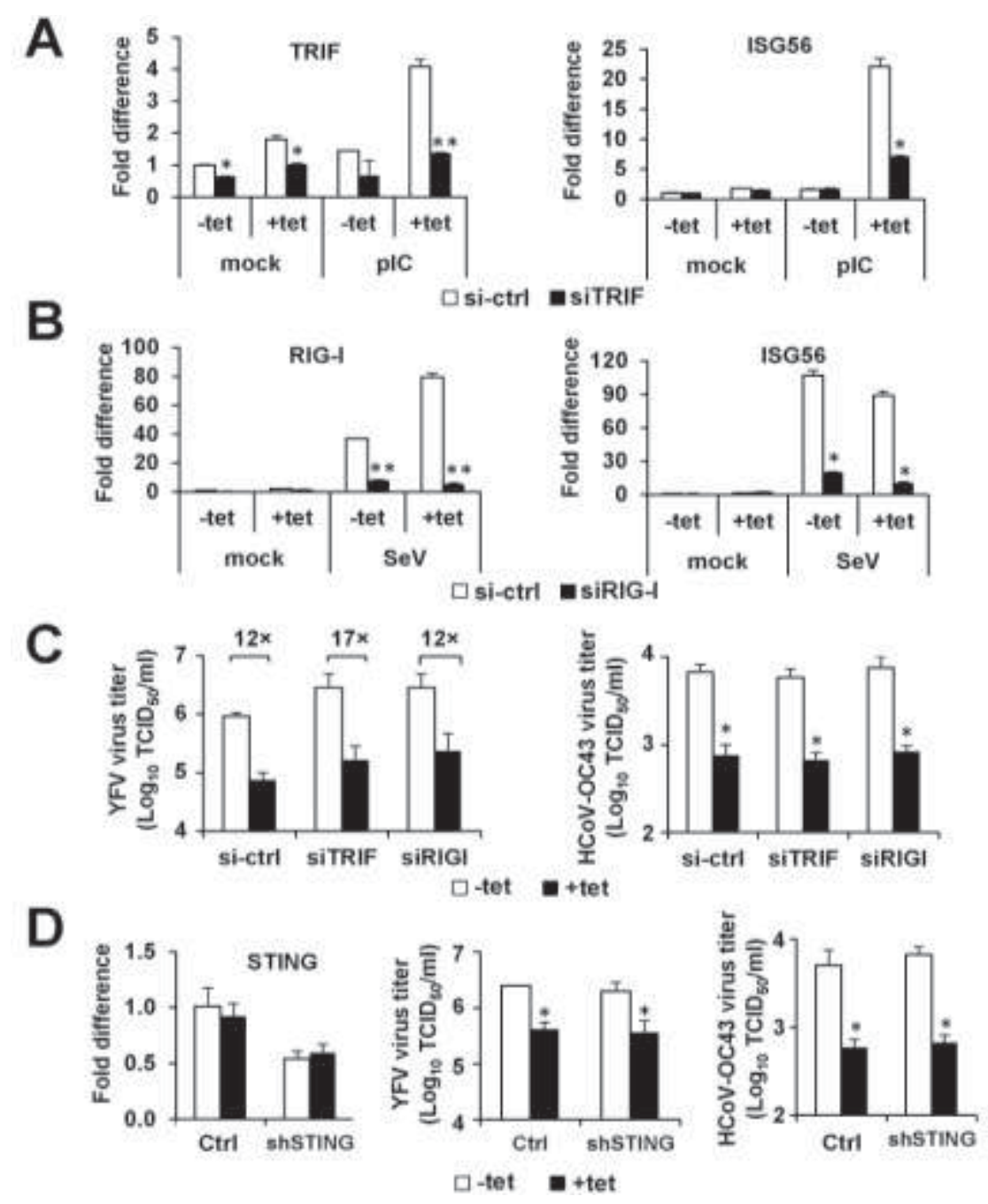

Figure 3-4. TRIM56's antiviral actions are independent of TRIF, RIG-I and STING.

HEK293-FIT-T56 cells cultured without (-tet) or with tet (+tet) were transfected with a negative control siRNA (si-ctrl) or siRNA targeting TRIF or RIG-I, or shRNA targeting STING (shSTING) or its control vector (Ctrl). At $48 \mathrm{~h}$ post transfection, the cells were mock-treated, infected with YFV-17D or HCoV-OC43 for $24 \mathrm{~h}(\mathrm{MOI}=0.1)$, or treated with poly-I:C (pIC) or Sendai virus (SeV) for $10 \mathrm{~h}$. qPCR was performed with total cellular RNA to measure abundance of TRIF and ISG56 mRNAs before or after pIC treatment in (A), or that of RIG-I and ISG56 mRNAs before and following SeV infection in (B). (C) Progeny virus production was determined in cell-free supernatants following silencing TRIF or RIG-I and infection of YFV-17D (left) or HCoV-OC43 (right). (D) Left panel shows qRT-PCR analysis of the abundance of STING transcript after transfection of control vector or shSTING. Progeny virus production was determined in cell-free supernatants following transfection of control vector or shSTING and infection with YFV-17D (middle) or HCoV-OC43 (right). "*”" and "**" denote that statistical differences exist between -tet and + tet cells (C and D), or between ctrl and knockdown cells (A and B) with a $P$ value of $<0.05$ and $<0.01$, respectively. 
signaling by interacting with TRIF (39). Also in agreement with our previous finding that TRIM56 has no appreciable role in RIG-I signaling (39), tet-induction of HA-TRIM56 had no effect on upregulation of ISG56 mRNA by Sendai virus infection, which was blunted by RIG-I siRNA (Figure 3-4B). Despite efficient knockdown of TRIF or RIG-I, TRIM56's antiviral activities against YFV and $\mathrm{HCoV}-\mathrm{OC} 43$ remained unchanged (Figure 3-4C). I also did not find knockdown of STING alters TRIM56's suppressive effects on YFV or HCoV-OC43 multiplication (Figure 3-4D).

\section{Development of HEK293 Cells with Conditional Expression of Various TRIM56 Mutants for Mapping of the Antiviral Determinants}

In order to dissect the mechanisms by which TRIM56 exerts its antiviral actions against different RNA viruses, it was necessary to define the domains/activities critical for TRIM56's restriction of each virus. Thus, I established HEK293 cell lines with tetinducible expression of a series of HA-TRIM56 mutants using the strategy we adopted to generate the 293-FIT-T56 cell line. These included the E3 Ub ligase-deficient CC21/24AA mutant (referred to as CC21/24AA), mutants lacking the RING (referred to as $\triangle$ RING), B-box (referred to as $\Delta \mathrm{B}$-box) or coiled-coil (referred to as $\Delta$ Coiled-coil) domains, respectively, and mutants bearing deletions in the C-terminal portion $-\triangle 355$ 433 (lacking aa 355-433), $\Delta 621-695$ (lacking aa 621-695), and $\Delta 693-750$ (lacking aa 693-750). As shown in Figure 3-5, there was negligible expression of WT and mutant HA-TRIM56 when these cell lines were cultured in tet-free medium (odd-numbered lanes except lane 17), while robust expression of HA-TRIM56 and its mutants (even-numbered lanes) with comparable levels and expected molecular masses was induced upon tet addition.

The E3 Ligase Activity and the Integrity of the C-terminus of TRIM56 Are Critical for the Antiviral Activity against YFV and DENV2, while the B-box Is Dispensable

Having created HEK293 cell lines with tet-regulated expression of various TRIM56 mutants, I used them in conjunction with the WT TRIM56-inducible 293-FIT-T56 cells to map the molecular determinants dictating TRIM56's antiviral activities against the three RNA viruses. First, I examined how YFV-17D replication differed among these cell lines. As shown in Figure 3-6A, tet induction of the $\triangle \mathrm{B}$-box mutant blunted YFV NS3 protein abundance (compare lanes 12 vs 11) in infected cells, acting with similar potency to that of WT TRIM56 (compare lanes 4 vs 3). In stark contrast, this inhibitory effect was absent when comparing cells expressing $\Delta$ RING, $\Delta 355-433, \Delta 621-695$ and $\Delta 693-750$ mutants with cells not induced to express (for $\Delta \mathrm{RING}$ mutant, compare lanes 24 vs 23 ; for $\Delta 355-433$ mutant, compare lanes 20 vs 19 ; for $\Delta 621-695$ mutant, compare lanes 28 vs 27; and for $\Delta 693-750$ mutant, compare lanes 32 vs 31 ). These data imply that the RING domain and the integrity of the $\mathrm{C}$-terminal parts are critical for the antiviral function of TRIM56 against YFV, while the B-box is dispensable. To determine the involvement of the E3 ligase activity, which lies in the RING-domain, I subjected the CC21/24AA 


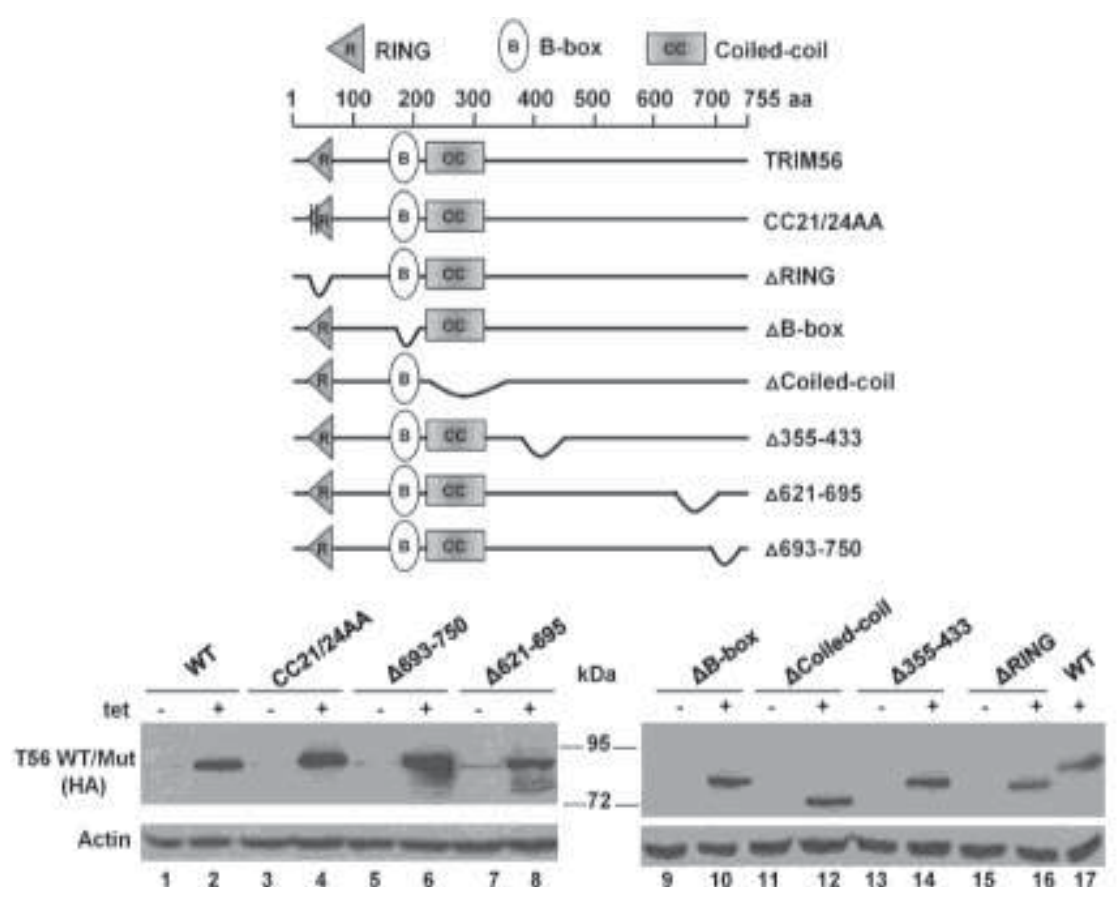

Figure 3-5. Characterization of HEK293 cells that conditionally express individual TRIM56 mutants in a tet-inducible manner.

Upper panel: Schematic representation of TRIM56 protein domains and the individual TRIM56 mutants investigated in this study. Lower panel: Immunoblot analysis of HATRIM56 and actin expression in 293-FIT-T56 and the indicated 293-FIT-T56 Mut cells with or without tet treatment for $48 \mathrm{~h}$. 


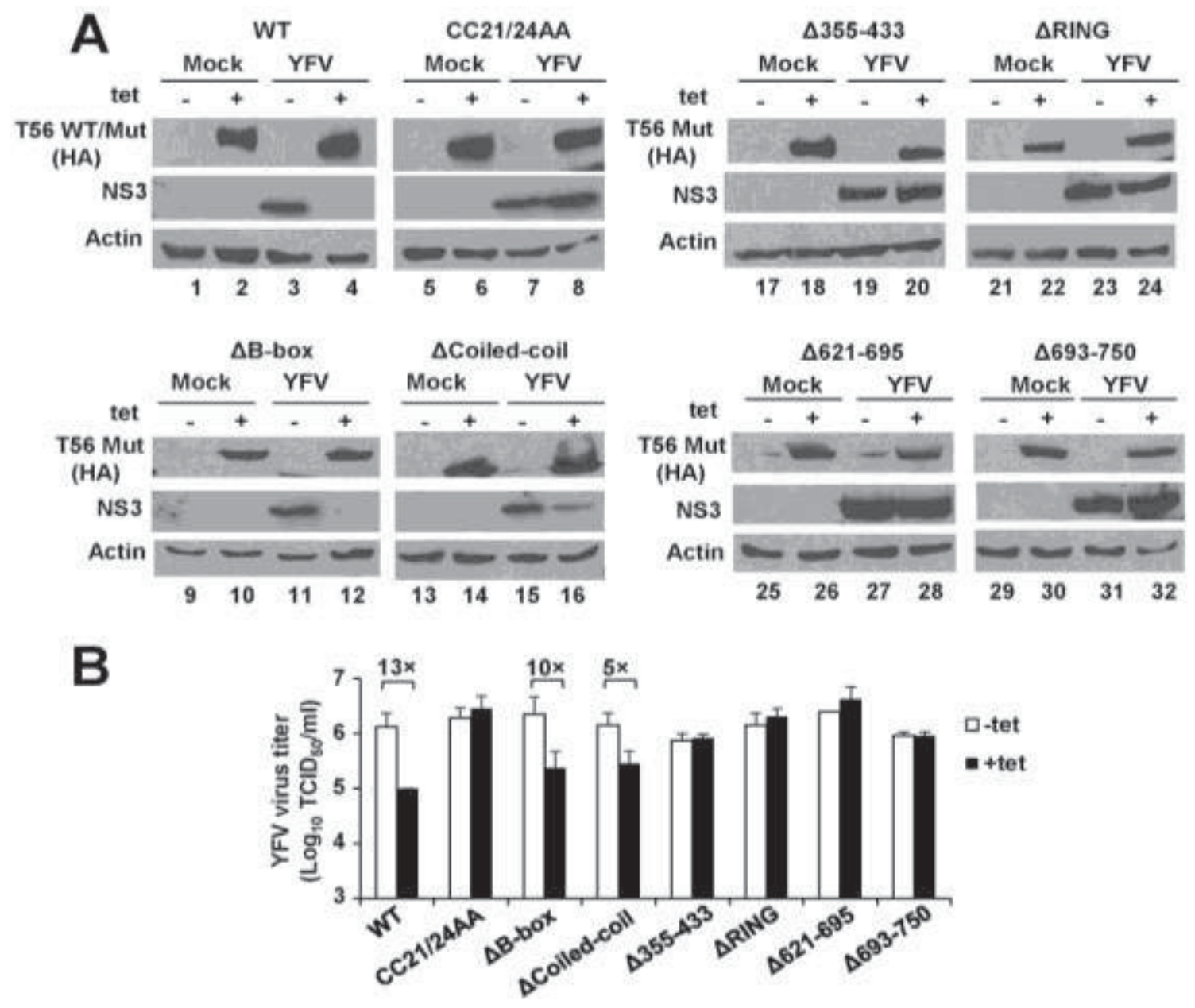

Figure 3-6. The E3 ligase activity and the integrity of the C-terminal parts of TRIM56 are essential for the antiviral activity against YFV.

(A) Immunoblot analysis of HA-tagged wild-type and mutant TRIM56 and YFV NS3 protein expression in 293-FIT-T56 WT/Mut cells in the absence (odd numbered lanes) or presence (even numbered lanes) of tet, and mock-infected or infected with YFV-17D for $24 \mathrm{~h}(\mathrm{MOI}=0.1)$. There were eight lines of 293-FIT-T56 WT/Mut cells examined in total, data from which are shown in eight blocks of immunoblot collections. (B) Progeny virus production in culture supernatants of 293-FIT-T56 WT/Mut cells cultured in the absence or presence of tet at $24 \mathrm{hpi}$ with YFV-17D $(\mathrm{MOI}=0.1)$. 
mutant inducible cells to YFV-17D infection. Tet induction of CC21/24AA mutant had no effect on YFV NS3 expression (compare lanes 8 vs 7), indicating that the E3 ligase activity plays a critical role in conferring the anti-YFV activity. This notion was additionally supported by data obtained from YFV-17D-infected 293-T56 and 293-T56CC21/24AA cells in which TRIM56-Flag or its CC21/24AA mutant version was expressed in a constitutive fashion (data not shown).

Consistent with the immunoblot results, there was a 10-13-fold decrease in progeny virus production in 293-FIT-T56 and 293-FIT-T56- $\Delta$ B-box cells in the induced state compared with cells cultured in the absence of tet. However, the decline in progeny viral yield upon tet induction was not seen in 293-FIT-T56-CC21/24AA, - $\triangle \mathrm{RING},-\Delta 355-433$, - $\Delta 621-695$ or $-\Delta 693-750$ mutant cells (Figure 3-6B). Notably, an intermediate antiviral phenotype was observed upon inducible expression of $\Delta$ Coiled-coil. While this mutant retained the ability to inhibit YFV-17D, it was less effective than WT TRIM56 or the $\triangle \mathrm{B}$-box mutant, as determined by immunoblotting of NS3 (Figure 3-6A, compare lanes 16 vs 15) and measurement of progeny virus production (Figure 3-6B). Thus, the coiledcoil domain is, to some extent, involved in the restriction of YFV by TRIM56, although the part this domain plays is not as crucial as those played by the E3 ligase activity and the C-terminal portions of the protein.

Next, I analyzed the molecular determinants that control the TRIM56 restriction of DENV2 using 293-FIT-T56 cells and our panel of mutant TRIM56 inducible cell lines. The TRIM56 domain involvement profile uncovered (Figure 3-7) was almost identical to that required for the anti-YFV action. Yet, there was one exception - the $\Delta$ Coiled-coil mutant still effectively inhibited the accumulation of DENV2 prM protein (Figure 3-7A, compare lanes 16 vs 15) and progeny virus production (Figure 3-7B), similar to those seen with WT TRIM56 or $\triangle \mathrm{B}$-box (Figure 3-7A-B), suggesting that unlike the case of YFV, the anti-DENV2 action is not strictly dependent on TRIM56's coiled-coil domain.

\section{The Functional TRIM56 Mutants Exert Their Antiviral Effects Independent of the Endogenous, WT TRIM56 Protein}

I recognize one caveat concerning the experiments probing the antiviral functions of the TRIM56 mutants. One cannot exclude the possibility that some of the mutants functioned by pairing with the endogenous WT TRIM56 protein, despite their expression levels in ectopic expression settings were $\sim 12$ fold higher than the latter (Figure 3-8B). However, this scenario is unlikely. The coiled-coil domain that is responsible for facilitating dimerization or oligomerization of TRIMs is dispensable for the antiviral activities of TRIM56 against DENV2 (Figure 3-7B) and HCoV-OC43 (Figure 3-9B, see the next section), except in the case of YFV (Figure 3-6B) it contributed to a minor extent. To confirm this notion, I conducted siRNA knockdown experiments to efficiently silence the endogenous TRIM56 mRNA (Figure 3-8A) without affecting the exogenously introduced mutant TRIM56 expression (Figure 3-8B), using an siRNA specifically targeting the 3'UTR region of the human TRIM56 transcript. As shown in Figure 3-8C, knockdown of endogenous WT TRIM56 by this siRNA did not reverse the 

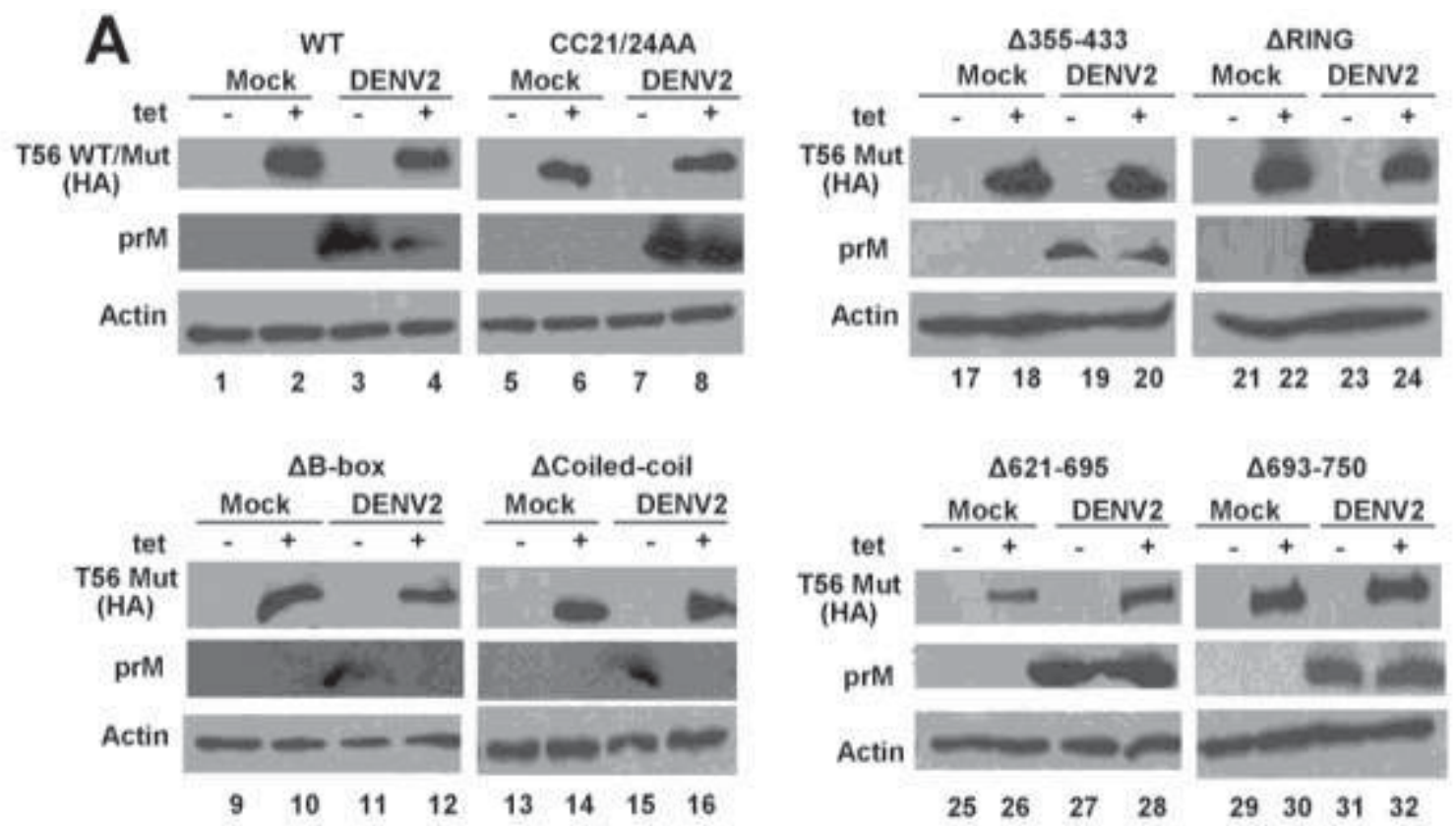

B

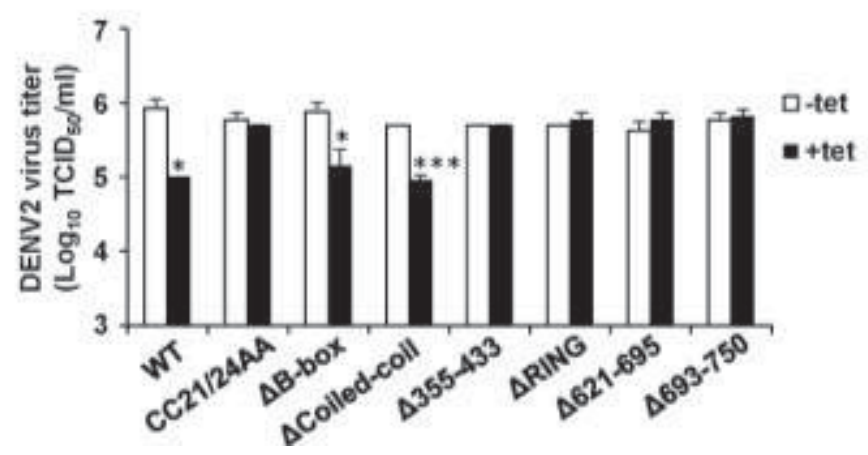

Figure 3-7. The suppression of DENV2 multiplication by TRIM56 requires its E3 ligase activity and the integrity of the $\mathrm{C}$-terminal portions.

(A) Immunoblot analysis of HA-TRIM56 (WT or mut) and DENV2 prM protein abundance (using goat anti-DENV2 antibody) in 293-FIT-T56 WT or individual Mut cells in the absence (odd numbered lanes) or presence (even numbered lanes) of tet, and mock-infected or infected with DENV2-16681 strain (MOI =1) for $60 \mathrm{~h}$. (B) Progeny virus production in culture supernatants of 293-FIT-T56 WT/Mut cells cultured in the absence or presence of tet at 60 hpi with DENV2-16681 strain $(\mathrm{MOI}=1)$. "**" and “***" indicate that statistical differences exist between -tet and +tet cells with a $P$ value of $<$ 0.05 and $<0.001$, respectively. 
A
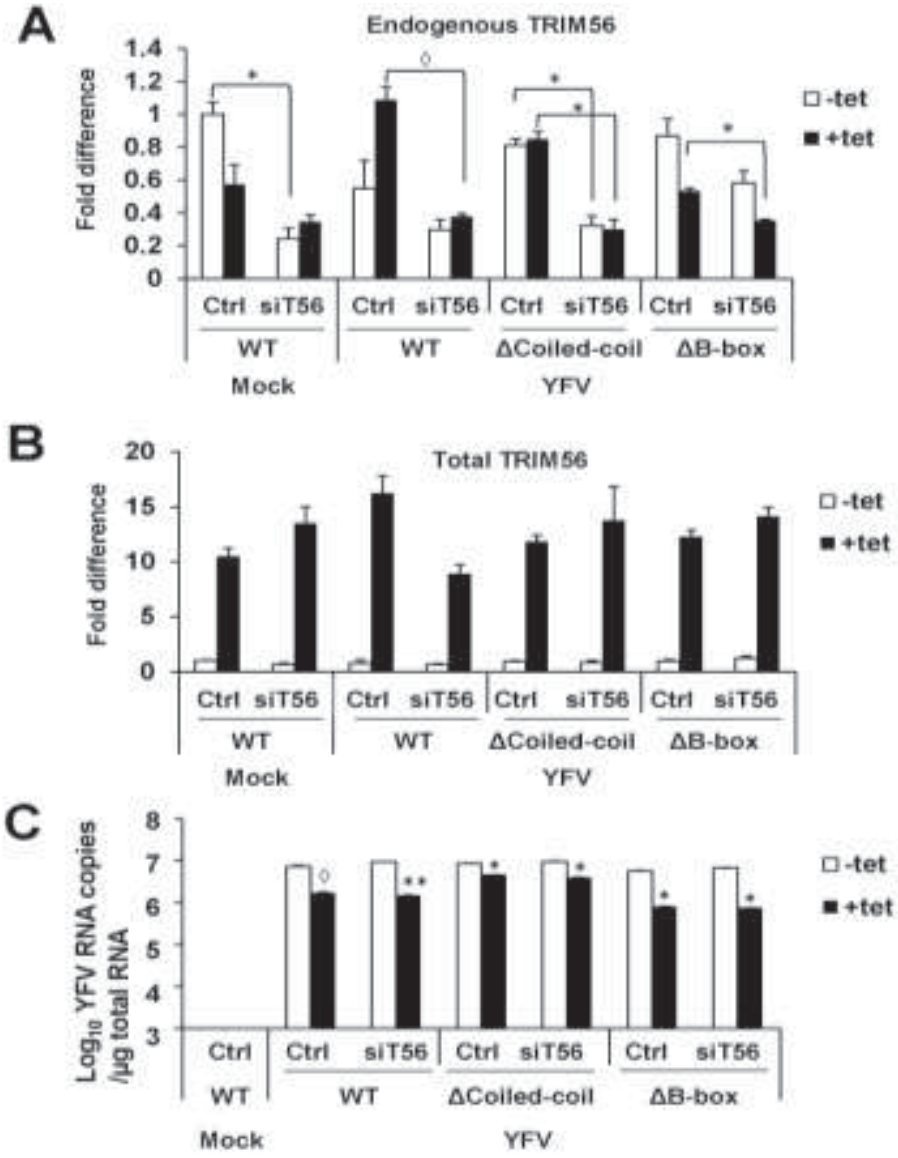

Figure 3-8. Knockdown of endogenous TRIM56 via an siRNA targeting the 3' UTR of human TRIM56 transcript does not affect the antiviral activities of the ectopically expressed WT TRIM56 or functional TRIM56 mutants. qRT-PCR was performed to measure the abundance of endogenous TRIM56 transcript (panel A, using primers amplifying the 3' UTR of TRIM56 mRNA), total TRIM56 transcript including the endogenous and exogenously overexpressed TRIM56 mRNAs (panel B, using primers amplifying the coding region of TRIM56 mRNA), and intracellular YFV RNA (panel C, using primers amplifying the NS2B region of YFV RNA) in 293-FIT-T56 (WT), 293-FIT-T56- $\triangle$ Coiled-coil and 293-FIT-T56- $\Delta$ B-box cells cultured with or without tet, and transfected with a negative control siRNA (Ctrl) or siRNA targeting 3' UTR of TRIM56 (siT56). Cell were either mock-infected or infected with YFV-17D for $24 \mathrm{~h}(\mathrm{MOI}=1)$. Note that the TRIM56-3'UTR siRNA only knocked down endogenous TRIM56 transcript but not the exogenously introduced (tet-inducible) WT or mutant TRIM56 which expressed only the coding region of the gene. (panel B); 3) the $\triangle$ Coiled-coil mutant inhibited YFV RNA replication less efficiently than WT TRIM56 or the $\Delta$ B-box mutant; and 4) the TRIM56-3'UTR siRNA had no demonstrable effects on the antiviral activity of any of the exogenously expressed TRIM56 forms. "*" and "**" denote that statistical differences exist between -tet and +tet cells (panel C), or between control and knockdown cells (panel A) with a $P$ value of $<0.05$ and $<0.01$, respectively. " $\diamond$ " denotes a $P$ value of $0.05-0.06$. 
significant dip in intracellular YFV RNA levels in 293-FIT-T56- $\Delta$ B-box or 293-FITT56- $\Delta$ Coiled-coil cells imposed by tet-induction of mutant TRIM56, suggesting that the antiviral effects of these mutants are not dependent upon the endogenous WT protein.

\section{TRIM56's Suppression of HCoV-OC43 Multiplication Depends on Its E3 Ligase Activity, but Not the Integrity of the C-terminus or B-box or Coiled-coil Domains}

To determine whether the findings with TRIM56 domain/activity requirements for its restriction of flaviviruses can be extended to HCoV-OC43, I compared the HEK293 tetinducible cell lines expressing WT or various TRIM56 mutants for their ability to support $\mathrm{HCoV}-\mathrm{OC} 43$ infection. I monitored expression of the viral structural $\mathrm{N}$ protein in cell lysates by western blotting (Figure 3-9A) and progeny viral titers in culture supernatants via $\mathrm{TCID}_{50}$ assay (Figure 3-9B). When infected with HCoV-OC43 at an MOI of 0.1, abundant expression of $\mathrm{N}$ protein was detected in all cell lines that were cultured in tetfree medium at 24 hpi. However, upon induction of WT TRIM56 or mutants lacking Bbox or the coiled-coil domain, or any of the C-terminal deletion mutants $(\Delta 355-433$, $\Delta 621-695$ and $\Delta 693-750$ ) by tet, $N$ protein abundance was compromised (Figure 3-9A). In contrast, there was no reduction in the expression level of this viral protein when the tet-treated cells started to express the CC21/24AA or $\triangle \mathrm{RING}$ mutants, both of which are defective for E3 ligase activity (Figure 3-9A, compare lanes 8 vs 7 and lanes 24 vs 23, respectively). Analysis of progeny virus production confirmed that all TRIM56 mutants but $\mathrm{CC} 21 / 24 \mathrm{AA}$ and $\triangle \mathrm{RING}$ retained the ability to curtail $\mathrm{HCoV}-\mathrm{OC} 43$ propagation (Figure 3-9B). Taken together, these observations demonstrate that the E3 ligase activity alone, and not other domains/portions of TRIM56, mediates the anti-HCoV-OC43effect.

\section{TRIM56 Inhibits YFV/DENV2 Propagation by Impairing Intracellular Viral RNA Replication, whereas It Curbs $\mathrm{HCoV}-\mathrm{OC} 43$ Spread by Targeting a Later Step in the Viral Life Cycle}

Because TRIM56's antiviral effects against flaviviruses and $\mathrm{HCoV}-\mathrm{OC} 43$ share a dependence on the $\mathrm{E} 3$ ligase activity but differ in the requirement of the $\mathrm{C}$-terminal regions, I wondered to what extent their mechanisms of actions overlapped. Thus, I determined the effects of TRIM56 overexpression on different stages of YFV-17D (the prototype of flaviviruses) and $\mathrm{HCoV}-\mathrm{OC} 43$ life cycles. Three cell lines were included in the analysis: 293-FIT-T56-WT, 293-FIT-T56-CC21/24AA, and 293-FIT-T56- $621-695$. Cells were cultured in the absence or presence of tet to repress or induce TRIM56 (or mutant TRIM56) expression, respectively, followed by infection with YFV-17D (Figure 3-10A) or HCoV-OC43 (Figure 3-10B) at an MOI of 1. At 1, 12 and $24 \mathrm{hpi,}$ after extensive washes to remove extracellular virus and viral RNAs, cells were lysed for total RNA extraction, followed by measurement of intracellular viral RNA levels by qPCR. Parallel assays of viral titers in culture supernatants at 24 hpi confirmed that induction of TRIM56 but not CC21/24AA or $\Delta 621-695$ mutants significantly decreased progeny YFV-17D yield, and that expression of WT TRIM56 or $\Delta 621-695$ but not 

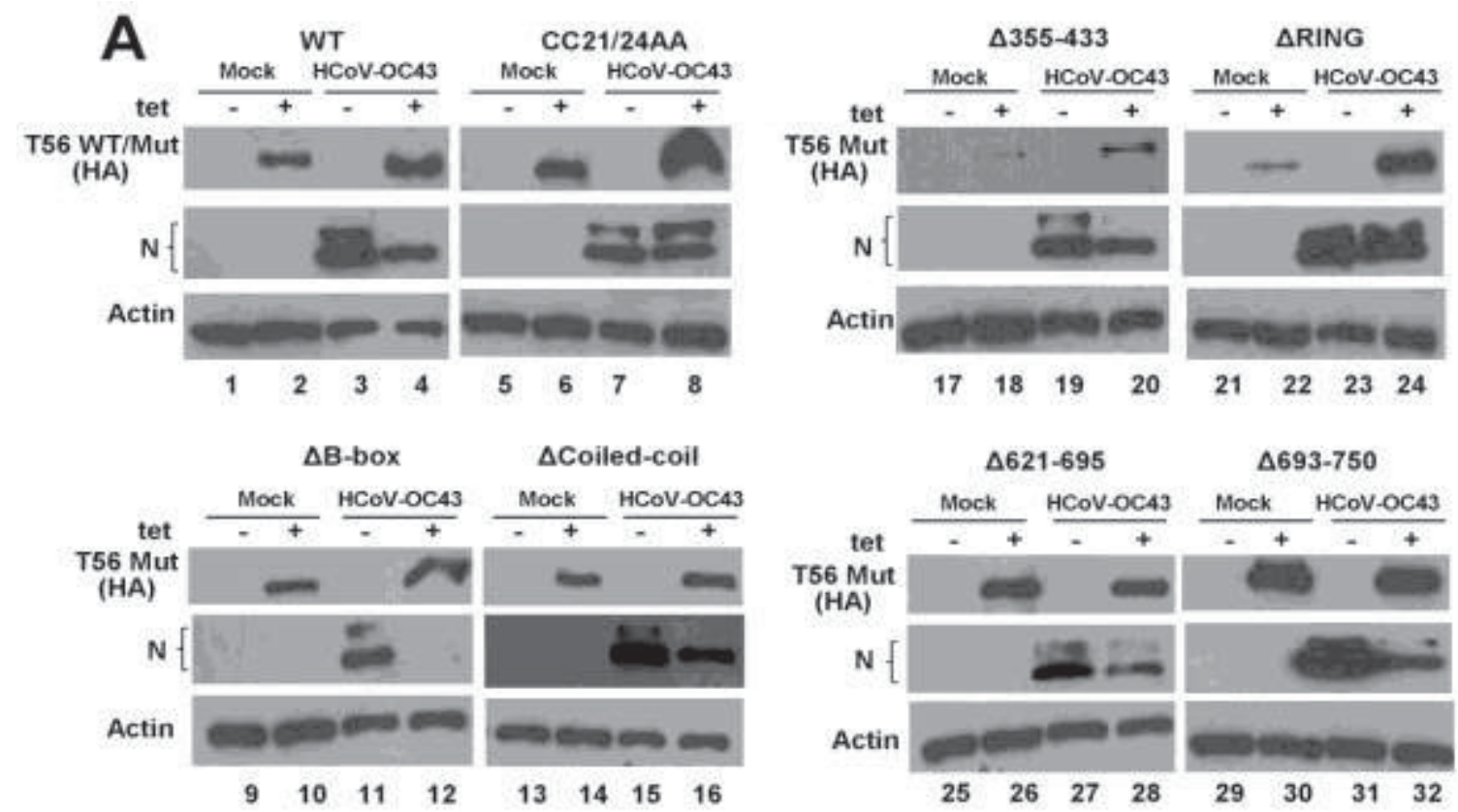

B

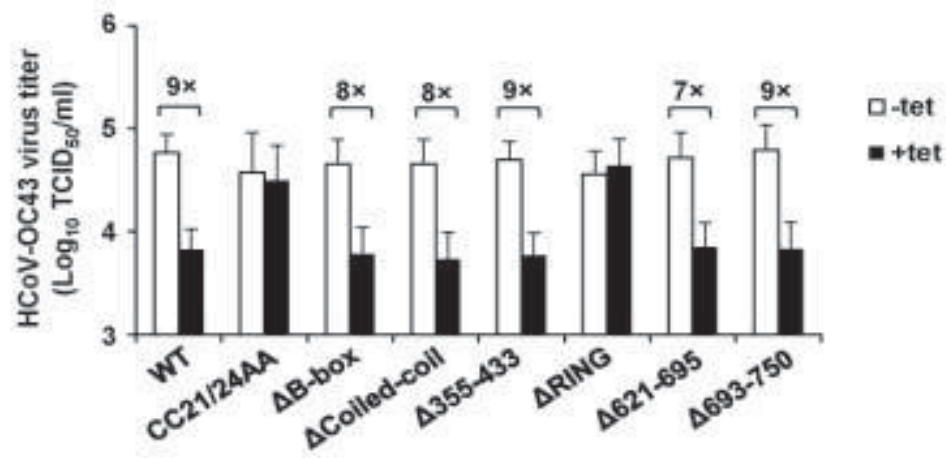

Figure 3-9. The inhibition of $\mathrm{HCoV}-\mathrm{OC} 43$ propagation by TRIM56 is dependent upon its E3 ligase activity, but not upon the integrity of the C-terminal portions, or the B-box or coiled-coil domains.

(A) Immunoblot analysis of HA-TRIM56 (WT or mut) and $\mathrm{HCoV}-\mathrm{OC} 43 \mathrm{~N}$ protein $(\mathrm{N})$ abundance in 293-FIT-T56 WT or individual Mut cells in the absence (odd numbered lanes) or presence (even numbered lanes) of tet, and mock-infected or infected with $\mathrm{HCoV}-\mathrm{OC} 43(\mathrm{MOI}=0.1)$ for $24 \mathrm{~h}$. (B) Progeny virus production in culture supernatants of 293-FIT-T56 WT/Mut cells in the absence or presence of tet at 24 hpi with HCoVOC43 $(\mathrm{MOI}=0.1)$. 

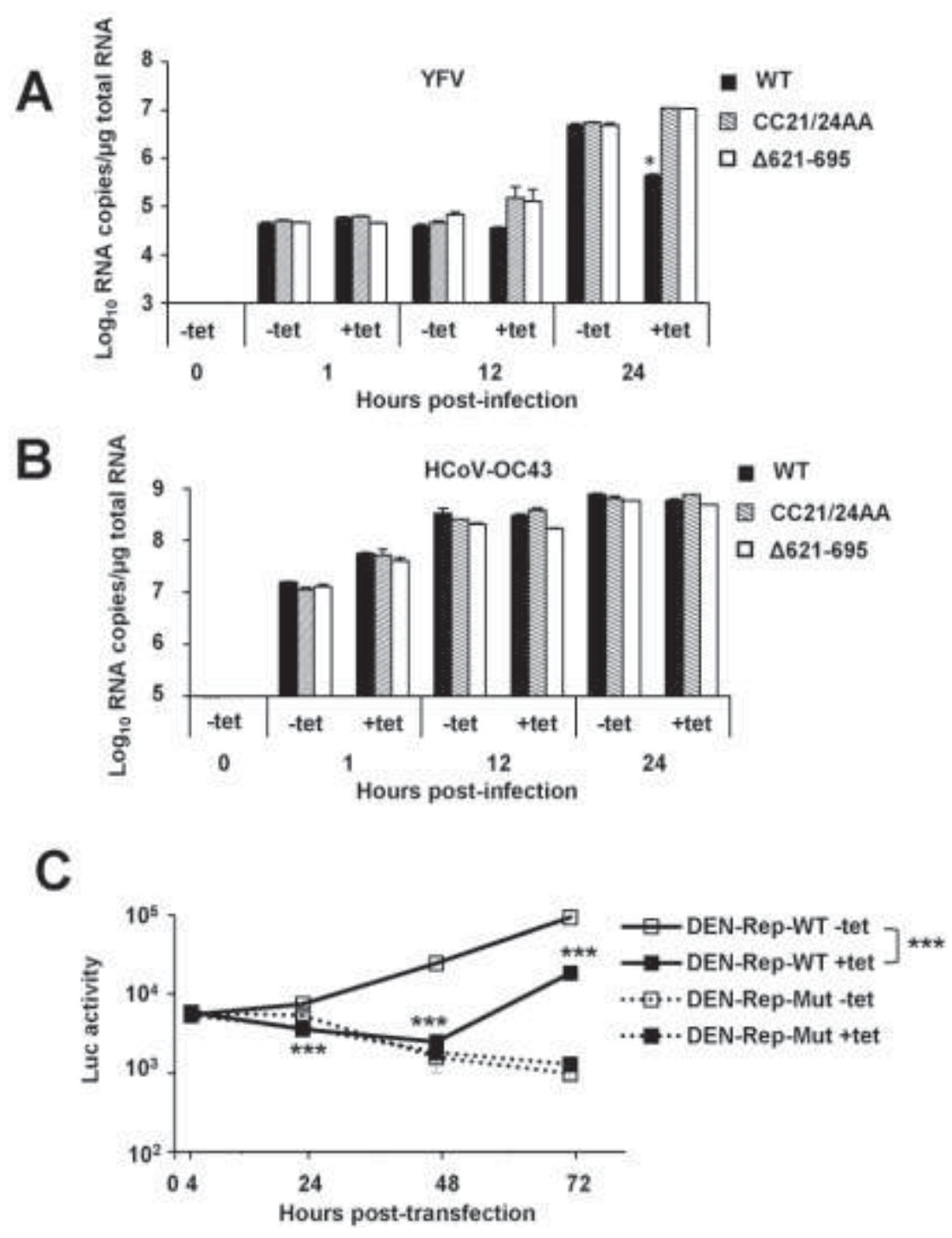

Figure 3-10. TRIM56 blunts YFV and DENV2 infection by targeting intracellular viral RNA replication, but inhibits $\mathrm{HCoV}-\mathrm{OC} 43$ propagation by acting on ater step in the viral life cycle.

(A) qRT-PCR analysis of intracellular YFV RNA abundance in 293-FIT-T56 (WT), CC21/24AA or $-\triangle 621-695$ cells cultured in the absence or presence of tet, and mockinfected or infected with YFV-17D $(\mathrm{MOI}=1)$ for 1,12 and $24 \mathrm{~h}$, respectively. (B) qRTPCR analysis of intracellular HCoV-OC43 N RNA abundance in 293-FIT-T56 (WT), CC21/24AA or $-\triangle 621-695$ cells cultured in the absence or presence of tet, and mockinfected or infected with $\mathrm{HCoV}-\mathrm{OC} 43(\mathrm{MOI}=1)$ for 1,12 and $24 \mathrm{~h}$, respectively. $(\mathrm{C})$ 293-FIT-T56 cells induced (+tet) or repressed (-tet) for HA-TRIM56 expression were transfected with WT or the replication-defective NS4B P104R mutant DENV2-TSV01 replicon RNA (designated as DEN-Rep-WT or -Mut). At indicated time points post transfection, cells were lysed and assayed for Renilla luciferase activity as readout of DENV RNA replication. "**" and “***” indicate that statistical differences exist between -tet and +tet cells with a $P$ value of $<0.05$ and $<0.001$, respectively. 
CC21/24AA led to a significant dip in HCoV-OC43 titers (data not shown). At 1 hpi, intracellular viral RNA levels for YFV-17D or HCoV-OC43 were indistinguishable between cells cultured without and with tet, regardless of the cell line examined (bearing WT or mutant TRIM56), suggesting that TRIM56 does not impede virus-receptor interaction or early cellular entry of either virus.

However, as infection progressed a different picture regarding the effects of TRIM56 emerged between the two viruses. In the case of YFV-17D (Figure 3-10A), intracellular viral RNA abundance peaked at 24 hpi, with no significant further increase at 36 hpi (data not shown), and was comparable among all three cell lines cultured in tet-free medium (without WT or mutant HA-TRIM56 induction). However, the peak level of viral RNA in tet-induced 293-FIT-T56 cells was 1 log lower than in uninduced cells, proportional to the difference in progeny viral titers. This implies that TRIM56 blunts YFV infection by targeting intracellular viral RNA replication. In contrast, 293-FIT-T56CC21/24AA and - $-621-695$ cells in induced state harbored more viral RNA than those cultured in the absence of tet, a phenomenon more appreciable at 12 than 24 hpi. The moderate increase in viral replication in these cells may reflect a dominant-negative effect of these TRIM56 mutants on endogenous WT TRIM56. In contrast to what was observed with YFV, in cells infected with HCoV-OC43 (Figure 3-10B), copy numbers of intracellular viral N RNA reached plateau at $12 \mathrm{hpi}$, and there was no difference among 293-FIT-T56-WT, -CC21/24AA or - $4621-695$ cells irrespective of tet treatment. Collectively, the significant decline in progeny HCoV-OC43 titers in 293-FIT-T56-WT and $-\triangle 621-695$ cells in induced state does not arise from impaired viral entry or RNA replication but rather is attributed to effect(s) of the TRIM56 E3 ligase on a later step in the viral life cycle, most likely virus packaging and/or egress.

To corroborate that TRIM56 curbs flaviviral RNA replication, I performed transient viral RNA replication assay in 293-FIT-T56 cells using WT or a replication-defective mutant (NS4B P104R) DENV2 replicon encoding Renilla luciferase. At 4 h post transfection, there was no difference in luciferase activity in cells transfected with either WT or mutant replicon, regardless of HA-TRIM56 expression status, suggesting little impact of TRIM56 on DENV2 RNA translation (Figure 3-10C). However, different replication kinetics of the WT replicon were observed between +tet and -tet cells at later time points. Specifically, in cells repressed for HA-TRIM56 expression (-tet), the WT DENV2 replicon started to replicate at $24 \mathrm{~h}$ post transfection and climbed progressively in the next 2 days ( 48 and 72 h, Figure 3-10C). In stark contrast, in cells with induced HA-TRIM56 (+tet), the WT replicon did not replicate in the first $48 \mathrm{~h}$. Although this replicon began to replicate at $72 \mathrm{~h}$, its replication level, as gauged by luciferase activity, was significantly lower than that in -tet cells. As a negative control, the replicationdeficient NS4B mutant replicon failed to synthesize viral RNAs (41) and displayed a continuous decline in luciferase activity, regardless of HA-TRIM56 expression status (i.e., -tet or +tet) (Figure 3-10C, dashed lines). These data confirm that TRIM56 acts on the viral RNA replication step of DENV2 infection. 


\section{Discussion}

In this study, I have examined the roles of human TRIM56 in acting as an antiviral host factor against diverse positive-strand RNA viruses. Our data demonstrate that TRIM56 bears antiviral activities against two medically-important flaviviruses (YFV and DENV2) and extend the antiviral spectrum of this TRIM to include a human coronavirus (HCoV-OC43). Not only did I show that ectopic expression of TRIM56 put a check on these viruses (Figure 3-1), but our data obtained from cells depleted for the endogenous protein confirmed that physiologic levels of TRIM56 posed an antiviral barrier (Figure 3-2). No less important, the effects were reproduced in multiple cell types and regardless of the expression system utilized. Of note, preceded by demonstration of an inhibitory effect of TRIM56 on a ruminant pathogen, BVDV (34), the novel findings in the present study, for the first time, show that human TRIM56 functions as a versatile antiviral restriction factor of human-pathogenic positive-strand RNA viruses. Moreover, this study has delineated the contributions of TRIM56's B-box and coiled-coil domains as well as the entire $\mathrm{C}$-terminal portion to its novel antiviral functions. The full picture of the molecular determinants underlying the observed antiviral effects sheds light on the mechanisms of action of TRIM56.

We previously demonstrated that TRIM56 restricts the replication of BVDV (34). The close relatedness of DENV and YFV to BVDV is in line with their shared sensitivity to TRIM56's antiviral actions. However, a seemingly contradicting observation previously made was that TRIM56 failed to inhibit hepatitis $\mathrm{C}$ virus (HCV) replication when overexpressed in human hepatoma Huh7 cells (34), the current cell culture model permitting efficient propagation of this virus. Exactly why TRIM56 was inefficient in curbing HCV (which is classified in the Hepacivirus genus within the family Flaviviridae) is unknown. However, it is important to point out that Huh7 cells have severely impaired antiviral responses $(50,59)$. Perhaps one or more cellular cofactors required for TRIM56's antiviral actions are missing in this particular cell line. Indirectly supporting this possibility, our group has previously found that ISG20, an IFN-inducible exonuclease, acted as a potent inhibitor of YFV replication in HEK293 cells but had no demonstrable antiviral activity in Huh7.5 cells, a subline of Huh7 cells (49). Clearly, examining the effect of TRIM56 on HCV in other cell hosts is warranted. Along this line, our attempts to establish HCV RNA replicons in the tet-inducible 293-FIT-T56 cells, however, have thus far turned up fruitless for unknown reasons, although HEK293 cells had previously been reported to support some selectable HCV RNA replicons at very low efficiencies (60).

The discovery of TRIM56's antiviral activity against a human coronavirus substantially broadens our understanding of the antiviral spectrum of this TRIM. Classified within the family Coronaviridae, HCoV-OC43 belongs to the genus Betacoronavirus that is shared by two other highly pathogenic coronaviruses, severe acute respiratory syndrome coronavirus and the newly-emerged Middle East respiratory syndrome coronavirus (61). Coronaviruses differ remarkably from Flaviviridae members 
(e.g., YFV and DENV) in their transmission route, replication strategy and pathogenesis. The newly-uncovered broad antiviral spectrum of TRIM56 is consistent with its ubiquitous distribution across human tissues (34). Our novel findings raise the possibility that TRIM56 may also act to defend cells against other RNA viruses of significant public health concern.

TRIM56 is among a handful of TRIMs that were recently shown to positively regulate innate antiviral pathways leading to IFNs production. Specifically, TRIM56 promotes the TLR3-dependent signaling pathway that recognizes viral double-stranded (ds) RNAs by forming a complex with TRIF, independent of its E3 ligase activity (39). TRIM56 also interacts with and activates STING, enhancing the cytosolic dsDNAsensing pathway $(39,58)$. However, data from the current study suggest a heightened IFN antiviral response does not underpin the antiviral effects of TRIM56 against YFV, DENV2 and HCoV-OC43 (Figure 3-3 and data not shown). Neither was it the case concerning the antiviral action of TRIM56 against BVDV (34). Consistent with IFNindependent antiviral mechanisms, overexpression of TRIM56 failed to inhibit VSV (34) and EMCV (Figure 3-1F), two viruses widely used for IFN bioactivity assays because of their extreme sensitivity to very low amount of IFNs. Moreover, siRNA knockdown experiments ruled out the involvement of TRIF-, RIG-I- or STING-dependent mechanisms in exerting the antiviral actions of TRIM56 (Figure 3-4). Despite the strong in vitro evidence summarized above suggesting direct antiviral effects of TRIM56, the possibility cannot be fully excluded that TRIM56 may promote innate immune signaling to these RNA viruses in primary cells and/or immune sentinel cells in vivo, contributing to viral clearance systematically. Future studies in TRIM56-deficient mice will be needed to elucidate the exact contributions of this TRIM to host antiviral defense. It should be noted, as well, given the growing evidence suggesting participation of the STINGdependent pathway in detecting DNA viruses and intracellular bacteria $(62,63)$, it will be of interest in future studies to investigate whether TRIM56 is also involved in cellular processes that fend off these classes of parasites.

Previous studies on other TRIMs have suggested that different TRIMs exert their antiviral effects via distinct mechanisms, which are dictated by different functional domains/portions. The N-terminal RING domain executes E3 ligase activity that promotes ubiquitination of viral proteins or host factors critical for viral replication, which are typically destined for proteasome-dependent degradation, as exemplified by TRIM22 E3 ligase-dependent degradation of influenza A virus nucleoprotein $(29,30,33)$. The B-box, coiled-coil domain and C-terminal portion (or the special domains lying in it), mediate protein-protein interactions (or protein-nucleic acid interactions which is also possible) that can perturb viral life cycles. Examples of this category include B-boxdependent TRIM15 restriction of murine leukemia virus (MLV) and the TRIM5 $\alpha$ Cterminal SPRY domain-mediated inhibition of human immunodeficiency virus (HIV) uncoating $(32,64)$. Remarkably, different domain involvements can be well orchestrated to perform TRIM-mediated antiviral functions. For instance, a model of TRIM E3 Ub ligase function has been proposed in which the RING domain bearing catalytically-active E3 ligase determines recognition of E2 conjugating enzyme meanwhile the coiled-coil domain and $\mathrm{C}$-terminal part ensure successful presentation of substrates to the E2 enzyme 
$(29,65)$. However, sometimes only one category of domain is involved in viral restriction. For example, TRIM15-mediated suppression of MLV requires its B-box domain alone, independent of the E3 ligase activity or the C-terminal SPRY domain (64). In the case of TRIM56, the overlapping and distinct domain requirement profiles unveiled in the current study may illuminate how TRIM56 renders cells resistant to three positive-strand RNA viruses from two distinct families.

TRIM56-mediated antiviral activities against YFV, DENV2 and HCoV-OC43 share the requirement for the RING domain (Figures 3-6, 3-7 and 3-9). However, they differ in the requirement of other molecular determinants. Specifically, while the E3 ligase activity was the only requirement for TRIM56-mediated anti-HCoV-OC43 action (Figure 3-9), the restriction of flaviviruses (YFV and DENV2) additionally depended on the integrity of the $\mathrm{C}$-terminal region of TRIM56 (Figures 3-6 and 3-7). When it comes to the coiled-coil domain, our data show that this motif is dispensable for restricting DENV2 and HCoV-OC43 (Figures 3-7 and 3-9), but contributes, to some extent, to antiYFV activity (Figure 3-6). Why there is a difference in the involvement of this domain for anti-YFV and anti-DENV2 actions is unknown and will require further study. Given that the coiled-coil domain is indispensable for homo- and hetero-dimerization of TRIMs (66), I postulate that TRIM56 may not absolutely rely on self-oligomerization or its pairing with another TRIM to curb infections by the RNA viruses investigated herein except YFV. Of note, I did not observe a dependence on the B-box domain for any of the TRIM56-mediated antiviral activities (Figures 3-6, 3-7 and 3-9), suggesting little contribution from B-box to the antiviral actions against flaviviruses and coronavirus. Although with structural similarity to the RING domain, B-box is not necessary for TRIM/E2 interaction (65). Altogether, the complex domain requirement profiles revealed for TRIM56-mediated inhibition of distinct RNA viruses illustrate a remarkable example of host restriction factor evolution, and explain why this TRIM is able to protect cells from invasion by a broad range of positive-strand RNA viruses.

Last but not the least, I discovered that TRIM56 restricts flaviviruses and HCoVOC43 by targeting distinct stages of the viral life cycle. Time-course intracellular viral RNA quantification data (Figures 3-10A and 3-10B) suggest that virus-cell receptor interaction or early cellular entry of these viruses was not affected, while accumulation of intracellular viral RNAs during replication of YFV but not $\mathrm{HCoV}-\mathrm{OC} 43$ was retarded. Moreover, transient replicon assay using a DENV2 replicon confirmed that TRIM56 curbs flavivirus RNA replication but not translation (Figure 3-10C). The effect on YFV and DENV2 thus mirrored with those observed with TRIM56 on BVDV RNA replicons (34), suggesting that TRIM56 commonly targets viruses in the family Flaviviridae at the intracellular RNA replication step. However, I cannot fully exclude the impact, if any, of TRIM56 on virion transport or RNA uncoating of YFV or DENV2 immediately after the early entry step. By contrast, the data that progeny viral yield but not intracellular viral RNA abundance was inhibited by TRIM56 during HCoV-OC43 infection point to an effect on the viral packaging/release stages, which slows down coronaviral spread. Of note, it has been reported that mouse TRIM56 inhibits HIV release from cells (64), setting a precedent for the ability of TRIM56 to act on this late step of viral infections. Although the underlying molecular details remain to be elucidated, the distinct 
mechanisms of action TRIM56 adopts to inhibit flaviviruses and HCoV-OC43 are consistent with the differential requirements of the $\mathrm{C}$-terminal portions of this TRIM. 


\section{CHAPTER 4. THE C-TERMINAL TAIL OF TRIM56 DICTATES ANTIVIRAL RESTRICTION OF INFLUENZA A AND B VIRUSES BY IMPEDING VIRAL RNA SYNTHESIS ${ }^{1}$}

\section{Introduction}

Classified within the family Orthomyxoviridae, influenza A and B viruses (IAV and IBV) are important respiratory pathogens responsible for significant morbidity and mortality worldwide (12-15). These viruses possess segmented, negative-strand RNA genomes that are encapsidated with nucleoprotein (NP) and bound with polymerase proteins, collectively known as viral ribonucleoprotein (vRNP) complexes $(14,15)$. One striking feature of the influenza virus life cycle is that virus RNA (vRNA) synthesis takes place in the nucleus $(15,20,21)$. After virion entry, trafficking of incoming vRNPs into the nucleus is an active process dependent on the nuclear import machinery, followed by initiation of virus transcription and replication (15). As is the case of most RNA viruses, replication of influenza viruses is error-prone, generating mutant viruses that can escape host immune responses and/or antiviral drugs. Despite intense research and antiviral drug development efforts, options to combat influenza are for the most part limited to those targeting the viral NA and M2 ion channel, and emergence of drug-resistant viral mutants is a pressing issue (15). Thus, studies on novel virus-host interactions that alter influenza virus fitness are warranted and may reveal new targets for therapeutic interventions.

In response to virus assaults including infection by influenza viruses, host cells employ exquisitely intrinsic mechanisms to fend off the invader. The induction of type I and type III inteferons (IFNs) represents a major immediate antiviral response in mammalian cells and is initiated by several classes of pattern recognition receptors (PRRs) including the Toll-like receptors (TLRs) and retinoic-inducible gene-I (RIG-I)like receptors (1-4). These PRRs engage viral pathogen-associated molecular patterns, most notably viral RNAs, triggering signaling cascades that culminate in activation of two critical transcription factors -- interferon regulatory factor 3 (IRF3) and NF- $\kappa B$, which play pivotal roles in the transcription of interferons and pro-inflammatory cytokines/chemokines, respectively (1-4). To circumvent this host defense mechanism, IAV blocks IRF3 activation by its multifunctional non-structural protein (NS)-1 to cease IFN production whereas it exploits activated NF- $\kappa$ B to regulate its viral RNA synthesis and promote inflammation $(25,26)$. In addition to the IFN system, host cells express, often constitutively, numerous antiviral proteins dubbed restriction factors, which act to curb different viral infections via distinct mechanisms (5). Among these, tripartite motifcontaining protein (TRIM) family members have recently garnered increasing attention, with several reported to participate in host defense against IAV (28-31).

\footnotetext{
${ }^{1}$ Adapted with permission. Liu B, Li NL, Shen Y, Bao X, Fabrizio T, Elbahesh H, Webby RJ, Li K. The C-terminal Tail of TRIM56 Dictates Antiviral Restriction of Influenza A and B Viruses by Impeding Viral RNA Synthesis. J Virol 2016. pii: JVI.03172-15.
} 
TRIM family proteins are characterized by a highly conserved N-terminal RBCC (ING- $\underline{B}$ box-Coiled coil) motif and are believed to possess E3 ligase activity conferred by the RING domain. In contrast, TRIM's C-terminal portions exhibit high variability in domain structures, based on which they are divided into up to 11 subfamilies (30,31). Of these, subfamily C-IV members, as exemplified by TRIM25 and TRIM22, harbor SPRY or/and PRY domains facilitating protein-protein interactions; TRIM-NHL proteins in

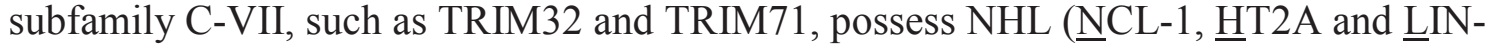
41)-repeats mediating binding to RNAs/proteins (67). Functionally, TRIMs have been implicated in a broad array of cellular processes that range from cell growth, differentiation, oncogenesis, and more recently, to immunity $(29,67-69)$. Of the immunity-regulating TRIMs reported to date, several play important roles in orchestrating PRR-mediated signaling cascades in response to viral invasions. A prime example is TRIM25, which promotes ubiquitination and activation of the PRR RIG-I to sense IAV infection and induce IFN expression (38). Acting in a different functional category, several TRIMs exhibit direct antiviral effects. For example, TRIM22 inhibits IAV by poly-ubiquitinating viral NP and targets it for proteasome-dependent degradation (33). Notably, both TRIM25 and TRIM22 depend on the RING-type E3 ligase activity to operate (29).

Recent evidence suggests that TRIM56 (also referred to as T56) is a novel player in innate antiviral immunity $(34,35,39)$. This TRIM protein belongs to the subfamily $\mathrm{C}-\mathrm{V}$ because it lacks a well-characterized domain in the C-terminal half hitherto $(28,29)$. TRIM56 promotes TLR3- and STING-mediated antiviral signaling pathways towards IFN induction via E3 Ub ligase-independent and -dependent mechanisms, respectively $(39,58)$. Additionally, TRIM56 exerts its direct antiviral effects, which are dependent on its E3 ligase activity, on four positive-strand RNA viruses, including three members of the family Flaviviridae, i.e., bovine viral diarrhea virus (BVDV), yellow fever virus (YFV) and dengue virus serotype 2 (DENV2), and a coronavirus, human coronavirusOC43 (HCoV-OC43) (34,35). However, little is known regarding TRIM56's antiviral spectrum against negative-strand RNA viruses, especially respiratory viruses such as influenza viruses, given the fact that TRIM56 is most abundantly expressed in the lung (34).

In this study I demonstrate that TRIM56 is an intrinsic host restriction factor of influenza viruses. Intriguingly, this attribute was found to be uncoupled from TRIM56's E3 ligase activity and the entire N-terminal TRIM motif was dispensable. Instead, the Cterminal 63 residues were both necessary and sufficient to inhibit replication of both IAV and IBV. Moreover, I show that TRIM56 impedes influenza virus infection by hindering viral RNA synthesis by its C-terminal tail portion. 


\section{Results}

\section{Ectopic Expression of TRIM56 Inhibits Propagation of IAV and IBV, but Not That of $\mathrm{SeV}$ or hMPV}

We had previously shown that TRIM56 is a restriction factor of four positive-strand RNA viruses, including three members of the Flaviviridae family (BVDV, YFV and DENV2) and a Coronaviridae family member (HCoV-OC43) (34,35). However, whether TRIM56 participates in host defense against negative-strand RNA viruses is largely unknown. VSV (a Rhabadovirus) is the only negative-strand RNA virus that has been examined to date; the propagation of this virus was not affected by ectopic expression of TRIM56 (34). In this study, I set out to determine if manipulation of TRIM56 abundance alters propagation of influenza viruses, which are medically important viruses classified within the family Orthomyxoviridae.

I first utilized HEK293-FIT-derived cells with tet-inducible expression of N-terminal HA-tagged TRIM56 (35) to examine the impact of TRIM56 overexpression on IAV. As shown in Figure 4-1A (left), negligible expression of HA-TRIM56 protein was observed in these cells when cultured in the absence of tet (-tet) but considerable HA-TRIM56 protein was detected following tet addition to culture medium (+tet) (compare lane $1 \mathrm{vs}$ 2 ). When infected with influenza $\mathrm{A} / \mathrm{PR} / 8 / 34$ virus at an MOI of 0.1 , there was abundant viral NP antigen in -tet cells at 9 hpi (lane 3). However, NP expression was diminished in cells induced for HA-TRIM56 expression (+tet, lane 4). Consistent with this, progeny virus titer in supernatants of 293-FIT-T56 + tet cells were significantly decreased as compared with -tet cells $(P<0.001)$ (Figure 4-1A, right). This antiviral effect of TRIM56 persisted at later time points (i.e., 16, 24 and 48 hpi) (Figure 4-1B). Additionally, MDBK-T56 cells stably expressing Flag-tagged human TRIM56 were substantially less susceptible to influenza A/PR/8/34 virus than MDBK-Bsr cells that express a control retroviral vector, as evidenced by substantially reduced intracellular NP antigen expression (Figure 4-1C, lower right immunoblot panels), $\sim 1-\log$ fewer $(P<$ 0.01 ) progeny virions yielded in culture supernatants (Figure 4-1C, upper right panel), and significantly lower percentage of NP-positive cells (Figure 4-1C, left immunostaining panel). Furthermore, TRIM56's antiviral effect against IAV was also observed in U2OS cells stably expressing TRIM56-Flag (U2OS-T56) compared with control cells (U2OS-Bsr) expressing a control vector (data not shown). To further corroborate this finding, I knocked down the ectopically expressed human TRIM56 in MDBK-T56 cells by lentiviral delivery of a TRIM56 shRNA (shT56). As shown in Figure 4-1D (lower immunoblot panel), TRIM56-Flag expression was reduced by $80 \%$ in the knockdown cells (MDBK-T56 shT56) compared with the control cells (MDBKT56 ctrl) transduced with a scrambled, negative control shRNA, concomitant with a 6fold increase $(P<0.05)$ in progeny virus production $(\mathrm{MOI}=0.5,24 \mathrm{hpi}$; Figure 4-1D, upper panel). Taken together, I conclude that ectopic expression of TRIM56 impedes IAV propagation. Importantly, this is a reproducible phenomenon, regardless of the expression system or cell type. 
Figure 4-1. Ectopic expression of TRIM56 hinders propagation of IAV/IBV, but not infection by hMPV or Sendai virus.

(A) (Left) HEK293-Flp-In T-REx-TRIM56 (293-FIT-T56) cells were cultured without (lanes 1 and 3, -tet) or with (lanes 2 and 4 , +tet) $(2 \mu \mathrm{g} / \mathrm{ml})$ for $48 \mathrm{~h}$, followed by mockinfection (lanes 1 and 2) or infection with influenza A/PR/8/34 (H1N1) virus for $9 \mathrm{~h}$ $(\mathrm{MOI}=0.1$; lanes 3 and 4). For +tet cells (lanes 2 and 4), they were kept in tet-containing media until lysed for western blot. Expression of N-terminally HA-tagged TRIM56 was detected using an anti-HA tag antibody (Invivogen) that does not cross-react with viral HA of H1N1 influenza A viruses and expression of IAV NP detected by an anti-influenza A/WSN/33 (H1N1) NP mAb. Actin served as a loading control to demonstrate equal sample loading. (Right) Progeny virus production in culture supernatants of 293-FIT-T56 cells with or without tet treatment at 9 hours post-infection (hpi) with influenza $\mathrm{A} / \mathrm{PR} / 8 / 34$ virus $(\mathrm{MOI}=0.1)$. (B) Progeny virus production in culture supernatants of 293-FIT-T56 cells cultured with or without tet at 0, 8, 16, 24 and 48 hpi with influenza $\mathrm{A} / \mathrm{PR} / 8 / 34$ virus $(\mathrm{MOI}=0.1)$. (C) (Left side) Immunofluorescence staining of NP (using anti-influenza A/WSN/33 (H1N1) NP mAb) in MDBK cells stably expressing TRIM56Flag (T56) or a control retroviral vector $(\mathrm{Bsr})$ at 24 hpi with $\mathrm{A} / \mathrm{PR} / 8 / 34$ virus $(\mathrm{MOI}=5)$. (Right side) Progeny virus titer in culture supernatants (upper) and NP and actin expression (lower) in lysates of infected cells under the same conditions as those on the left side. (D) (Lower) Immunoblot analysis of ectopically expressed human TRIM56 (using anti-TRIM56 antibody) in MDBK-T56 cells transduced with lentiviruses encoding either an shRNA specifically targeting human TRIM56 (MDBK-T56 shT56) or a nontargeting, scrambled control shRNA (MDBK-T56 Ctrl). (Upper) Progeny virus titers in culture supernatants of the indicated cells infected with influenza A/PR/8/34 virus for 24 $\mathrm{h}(\mathrm{MOI}=0.5)$. (E) (Upper) Progeny virus production in culture supernatants of 293-FITT56 cells with or without tet treatment at $24 \mathrm{hpi}$ with influenza B/Florida/4/06 (Yamagata) virus (MOI =0.1). (Lower) Immunoblot analysis of HA-tagged TRIM56 (using an anti-HA tag mAb (Invivogen) not cross-reacting with IBV HA) and viral HA (using an anti-influenza B/Hong Kong/8/73 HA pAb). Tubulin served as a loading control to demonstrate equal sample loading. (F) HeLa-FitA2-T56 cells with tet-regulated expression of HA-tagged TRIM56 were cultured with (lanes 3 and 4) or without tet (lanes 1 and 2), followed by mock-infection (lanes 1 and 3 ) or infection by hMPV (MOI = 1 , lanes 2 and 4) for $15 \mathrm{~h}$. Cell lysates were subjected to immunoblot analysis of HA-tagged TRIM56 (using anti-HA tag mAb (clone 12CA5, Roche)) and viral proteins (using antihMPV pAb). Asterisk denotes a nonspecific band. (G) 293-FIT-T56 cells cultured in the absence (-tet) or presence (+tet) of tetracycline were infected with Sendai virus $(\mathrm{SeV})$ at indicated doses $(1.6,16$ and $160 \mathrm{HAU} / \mathrm{ml})$ for $8 \mathrm{~h}$, followed by immunoblot analysis of HA-tagged TRIM56 (using anti-HA mAb (Invivogen)) and viral proteins (using anti-SeV pAb, which mainly reacted with $\mathrm{SeV}$ hemagglutinin-neuraminidase and nucleocapsid). Actin served as a loading control. Single, double and triple asterisks indicate that statistical differences exist between the indicated cells with a $P$ value of $<0.05,<0.01$ and $<0.001$, respectively. 


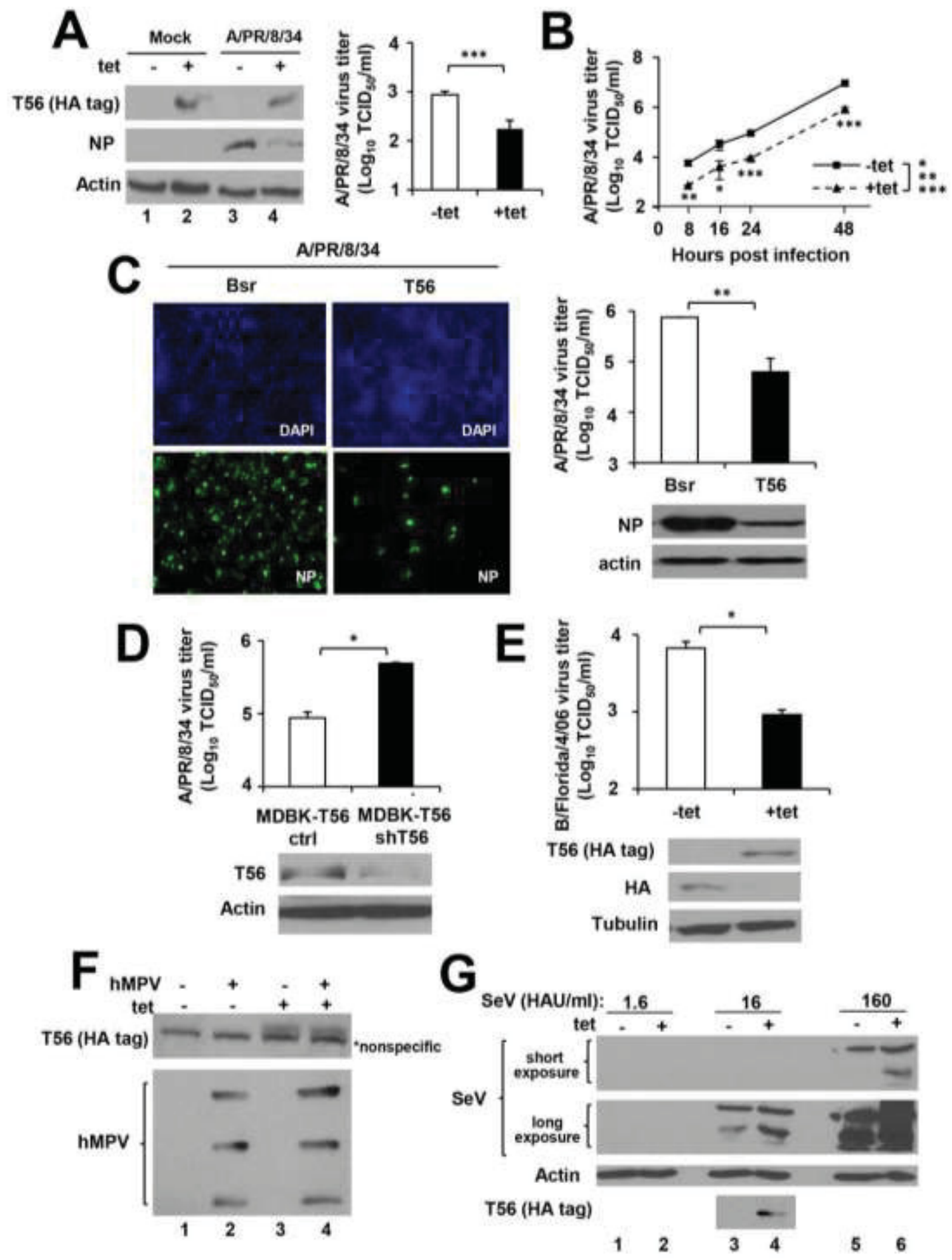


I next investigated if TRIM56's antiviral effect could be extended to IBV. It was found that induction of TRIM56 expression in 293-FIT-T56 cells led to a sharp decline in viral HA antigen abundance as compared with -tet (uninduced) cells at 24 hpi with influenza B/Florida/4/06 virus $(\mathrm{MOI}=0.1)$ (Figure 4-1E, lower panel). Concordant with this, progeny virus production in +tet cells was 7-fold $(P<0.05)$ lower than in -tet cells

(Figure 4-1E, upper panel). Similar anti-IBV effect of TRIM56 overexpression was also observed at 8 and 12 hpi $(\mathrm{MOI}=0.01)$ in HeLa-FitA2-T56 cells with tet-inducible expression of HA-TRIM56 (data not shown), again confirming that the phenomenon was not cell-type specific. Collectively, it was established that TRIM56, when ectopically expressed, curbs IBV infection. Of note, influenza viruses (IAV and IBV) represent the first negative-strand RNA virus family TRIM56 was found to inhibit.

To determine whether TRIM56 also restricts other negative-strand RNA viruses, I studied the effects of TRIM56 overexpression on hMPV (Figure 4-1F) and SeV (Figure 4-1G), paramyxoviruses that cause respiratory infections in humans and rodents, respectively. There was no inhibitory effect on viral protein expression following infection by either virus, when comparing cells induced for HA-TRIM56 expression and uninduced cells. The lack of an antiviral effect on these paramyxoviruses suggests that TRIM56 does not target negative-strand RNA viruses indiscriminately. Consistent with this concept, we previously demonstrated that TRIM56 overexpression does not inhibit VSV propagation (34).

\section{Knockdown of TRIM56 Augments Multiplication of Influenza Viruses}

To determine whether TRIM56 expressed at physiologic levels poses a barrier to infection by influenza viruses, I dissected the effect of TRIM56 knockdown on influenza virus propagation. Compared to control MDBK cells, MDBK-T56i cells with stable knockdown of endogenous TRIM56 (34; Figure 4-2A, left) supported substantially higher levels of viral NP expression and produced $>1$-log more $(P<0.01)$ progeny influenza A/PR/8/34 virions (Figure 4-2A, middle). In contrast, MDBK-T56i cells were not more permissive than control MDBK cells to infection by VSV-Luc, a recombinant VSV expressing luciferase as readout of viral replication (Figure 4-2A, right). These data lend further support to the notion that endogenous TRIM56 specifically hinders influenza virus infection. The same can be said regarding the impact of TRIM56 depletion on IAV infection in HeLa cells (Figure 4-2B). I found that HeLa cells with stable knockdown of TRIM56 (shT56) yielded $\sim 6$-fold $(P<0.01)$ more progeny IAV than control cells. HeLa-shT56 cells also supported higher levels of viral HA protein expression (Figure 4-2C, immunoblot panels) and $\sim 1-\log$ more $(P<0.01)$ progeny influenza virus production (Figure 4-2C, upper panel) than did control cells, following IBV infection. Altogether, these data demonstrate that TRIM56 expressed at physiological levels puts a check on infection by influenza A and B viruses. 

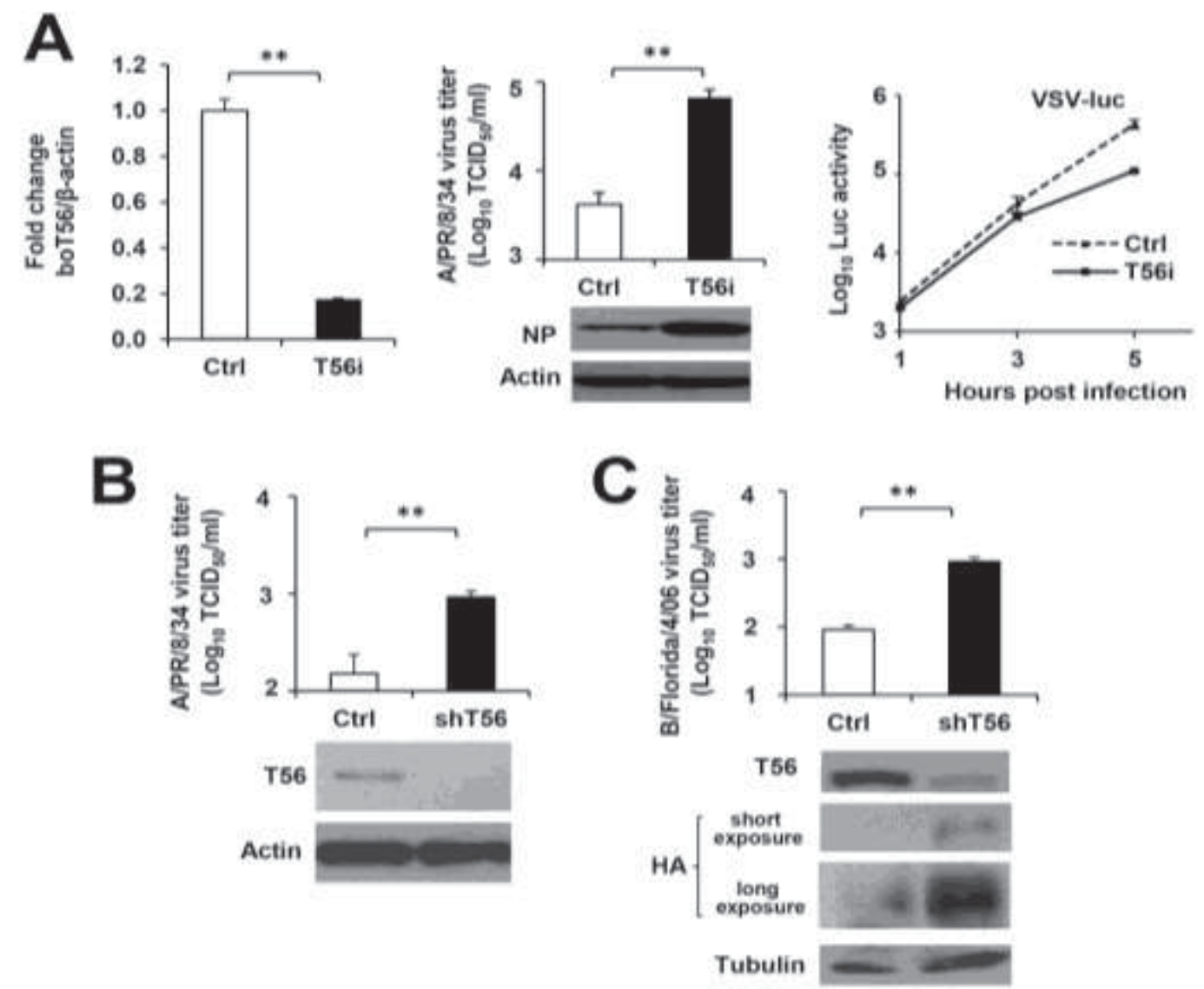

Figure 4-2. TRIM56 knockdown augments influenza virus multiplication.

(A) (Left part) qPCR analysis of bovine TRIM56 (boT56) mRNA expression in control MDBK cells (Ctrl) and its derived cells (T56i) stably transfected with a boT56 shRNA. (Upper, middle part) Progeny virus production in culture supernatants of MDBK-Ctrl and MDBK-T56i cells at 24 hpi with influenza A/PR/8/34 virus $(\mathrm{MOI}=5)$. (Lower, middle part) Immunoblot analysis of NP and actin expression in cell lysates under the conditions of the upper part. (Right part) MDBK-Ctrl cells and MDBK-T56i cells were infected with VSV-Luc at MOI of 0.2. At the indicated times, cells were lysed for firefly luciferase assay. (B) (Lower) Immunoblot analysis of human TRIM56 expression (using antiTRIM56 antibody) in HeLa-plkO.1 (Ctrl) cells stably transduced with a non-targeting, scrambled control shRNA or HeLa-shT56 (shT56) cells transduced with an shRNA specifically targeting TRIM56. (Upper) Progeny virus production in culture supernatants of the two cell lines at 8 hpi with influenza A/PR/8/34 virus (MOI =0.01). (C) (Lower) Immunoblot analysis of TRIM56 expression (using anti-TRIM56 antibody) and viral HA levels (using anti-influenza B/Hong Kong/8/73 HA pAb) in HeLa-plkO.1 (Ctrl) or HeLashT56 (shT56) cells at 8 hpi with influenza B/Florida/4/06 virus (MOI $=0.01)$. (Upper) Progeny virus production in culture supernatants of the cells under the same conditions as the lower immunoblot panel. Double asterisks indicate that statistical differences exist between the indicated cells with a $P$ value of $<0.01$. 


\section{The C-terminal Tail of TRIM56, but Not Its E3 Ligase Activity or the Other Portions of the Protein, Is Required for the Ability to Impede Influenza Virus Infection}

TRIM56 exerts antiviral actions against positive-strand RNA viruses of distinct families via overlapping and distinct molecular determinants $(34,35)$. Specifically, while the TRIM56's RING and the E3 ligase activity conferred by this domain are required to inhibit BVDV, flaviviruses and $\mathrm{HCoV}-\mathrm{OC} 43$, the C-terminal portion (residues 621-755) is critical for restriction of BVDV and flaviviruses but dispensable for HCoV-OC43 suppression $(34,35)$. To determine which domains or portions of TRIM56 are required for its novel antiviral actions against negative-strand RNA viruses, I mapped the TRIM56 molecular determinants which are essential for the restriction of influenza viruses, using HEK293-FIT-derived cell lines with tet-inducible expression of a series of HA-tagged TRIM56 mutants. The mutants included for analysis were the E3 Ub ligase-deficient CC21/24AA mutant, mutants lacking the RING ( $\triangle \mathrm{RING}), \mathrm{B}$-box ( $\Delta \mathrm{B}$-box) or Coiledcoil ( $\triangle$ Coiled-coil) domains, respectively, and mutants possessing different deletions in the C-terminal portion -- $\Delta 355-433, \Delta 621-695$ and $\Delta 693-750$ (Figure 4-3A, left). The expression levels of these mutants have been confirmed to be comparable to that of wildtype (WT) TRIM56 (35).

When challenged with the influenza A/PR/8/34 virus at MOI of 0.1 , an obvious inhibitory effect on influenza NP accumulation following tet induction (+tet) was observed at 9 or 27 hpi in HEK 293-FIT-T56 cells and in all of the mutant TRIM56inducible cells except the line that expresses the $\Delta 693-750$ mutant (Figure 4-3B). In agreement with the influenza NP immunoblot data, progeny virus production was curtailed by $\sim 1-\log$ by induction of WT TRIM56 $(P<0.001)$ or any of the TRIM56 mutant $(P<0.01)$, with the exception of the $\Delta 693-750$ mutant (Figure 4-3A, right). Thus, the very C-terminal tail portion of TRIM56 contains the sole determinant crucial for the anti-IAV activity. In stark contrast with what is required for flavivirus and coronavirus restriction, the E3 ligase activity of TRIM56 is dispensable for containing IAV.

To confirm the important findings which were shown above, I made effort to gauge influenza NP expression and progeny virus production in MDBK cells stably expressing Flag-tagged, WT or mutant human TRIM56 following infection by influenza A/PR/8/34 virus. As shown in Figure 4-3C (immuoblot data in lower panels), at 13 hpi (MOI = 3), abundant influenza NP antigen was detected in MDBK-Bsr cells expressing a control vector (lane 1). However, there was profound reduction in influenza NP expression in cells expressing WT TRIM56 (lane 3), the E3 Ub ligase-null CC21/24AA mutant (lane 4), $\triangle$ RING (lane 5) and $\Delta$ Coiled-coil (lane 6) mutants. In contrast, influenza NP expression was not reduced, but increased (lane 7) as compared with parental MDBK cells (lane 2). Progeny influenza virus titer data (Figure 4-3C, bar graph in upper panel) were in accordance with the influenza NP immunoblot results. Collectively, the data gleaned from MDBK-derived cells mirrored those obtained from HEK 293-FIT-derived cells. 
Figure 4-3. The restriction of IAV by TRIM56 relies on its C-terminal tail portion, but not on its E3 ligase activity or other parts of the protein.

(A) (Left) Schematic representation of TRIM56 protein domains and the individual TRIM56 mutants investigated in this study. (Right) Progeny virus production in culture supernatants of 293-FIT-T56-WT or individual TRIM56 mutant cells cultured in the absence (-tet) or presence (+tet) of tet at 9 hpi with influenza A/PR/8/34 virus $(\mathrm{MOI}=$ 0.1). (B) Immunoblot analysis of HA-tagged wild-type (WT) or mutant (mut) TRIM56 (using an anti-HA tag Ab (Invivogen) not reacting with viral HA of H1N1 IAV) and NP protein abundance (using anti-influenza A/WSN/33 (H1N1) NP mAb) in 293-FIT-T56 WT/Mut cells in the absence (odd numbered lanes) or presence (even numbered lanes) of tet, and mock-infected or infected with influenza A/PR/8/34 virus $(\mathrm{MOI}=0.1)$ for 9 or 27 h. Data from each cell line are presented in an immunoblot block ( 8 blocks in total, representing 8 different WT/mut TRIM56-inducible cell lines as shown in panel A). (C) (Lower) Immunoblot analysis of TRIM56 (using anti-human TRIM56 antibody) and NP protein abundance (using anti-influenza A/WSN/33 (H1N1) NP mAb) in parental MDBK as well as MDBK-derived cells stably expressing a control vector (Bsr), C-terminal Flagtagged human TRIM56 (WT) or its mutant versions infected with influenza A/PR/8/34 virus for $13 \mathrm{~h}(\mathrm{MOI}=3)$. (Upper) Progeny viral titers in culture supernatants of the indicated MDBK-derived cell lines infected with influenza $\mathrm{A} / \mathrm{PR} / 8 / 34$ virus $(\mathrm{MOI}=3)$ for $13 \mathrm{~h}$. Double and triple asterisks indicate that statistical differences exist between MDBK and WT/Mut T56 over-expressing cells, or between -tet and +tet cells with a $P$ value of $<0.01$ and $<0.001$, respectively. 
A

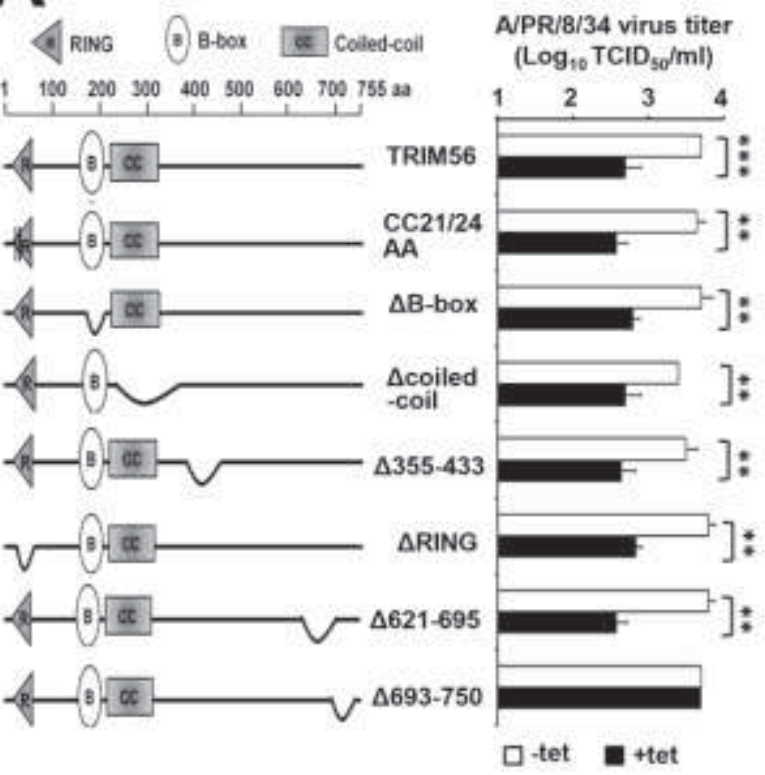

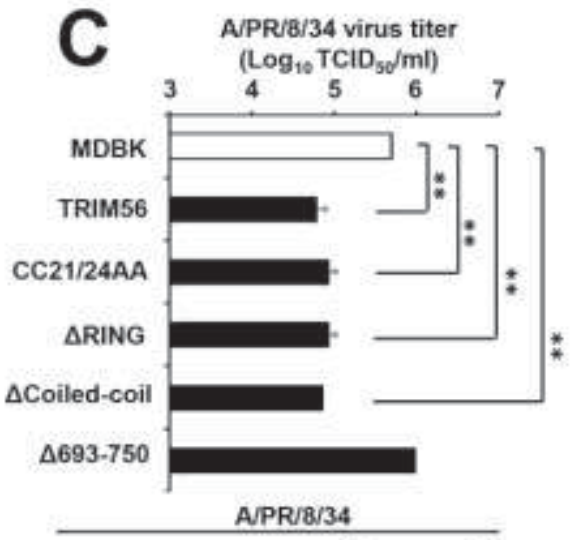

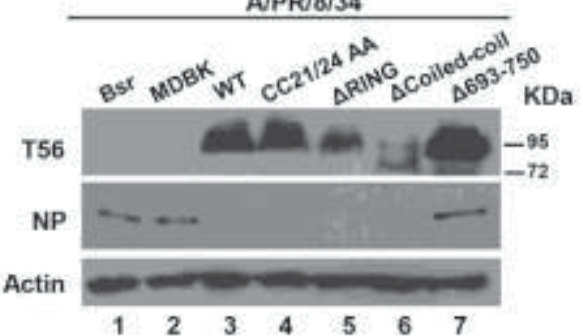

B NP f short exposure long exposure

Actin

tet

$\triangle B$-box (HA tag) NP. short exposure long exposure

Actin

tet

$\triangle 355-433$ (HA tag) NP $\left\{\begin{array}{c}\text { short exposure } \\ \text { long exposure }\end{array}\right.$

Actin

tet

ه621-695 (HA tag) NP $\left\{\begin{array}{c}\text { short exposure } \\ \text { long exposure }\end{array}\right.$

Actin $\frac{\text { Mock }}{-+} \frac{9 \mathrm{hpi}}{-+} \frac{27 \mathrm{hpi}}{-+}$
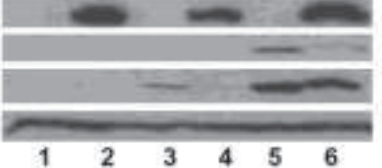

Mock $9 \mathrm{hpi} 27 \mathrm{hpi}$
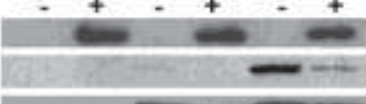

-

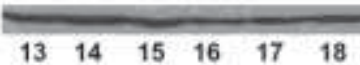

$$
\frac{\text { Mock }}{+} \frac{9 \mathrm{hpi}}{++} \frac{27 \mathrm{hpi}}{+}
$$
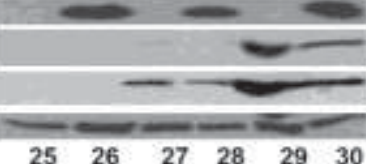

$\frac{\text { Mock }}{+} \frac{9 \mathrm{hpi}}{+}+\frac{27 \mathrm{hpi}}{+}$
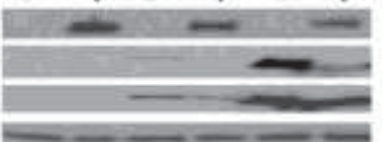

$\begin{array}{llllll}37 & 38 & 39 & 40 & 41 & 42\end{array}$ $\frac{\text { Mock }}{-+} \frac{9 \mathrm{hpi}}{-+} \frac{27 \mathrm{hpi}}{-+}$ tet

CC21/24AA (HA tag) short exposure ] NP long exposure $\mathrm{NP}$ Actin

$\begin{array}{llllll}7 & 8 & 9 & 10 & 11 & 12\end{array}$

$\frac{\text { Mock }}{+} \frac{9 \text { hpi }}{++} \frac{27 \text { hpi }}{+}$ tet $-\Delta$ Coiled-coil (HA tag)

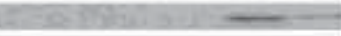

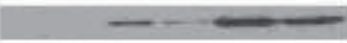
short exposure long exposure $f$ NP Actin

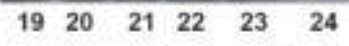

$\frac{\text { Mock }}{-+} \frac{9 \mathrm{hpi}}{-+} \frac{27 \mathrm{hpi}}{-+}$ tet

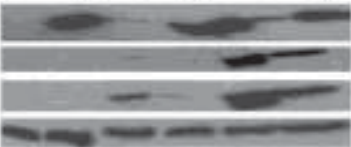

$\triangle R I N G$ (HA tag) short exposure If NP Actin

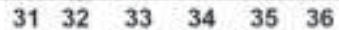

$\frac{\text { Mock }}{-+} \frac{9 \mathrm{hpi}}{-+} \frac{27 \mathrm{hpi}}{-+}$ tet

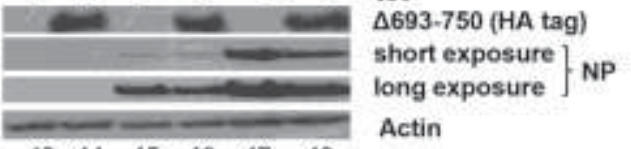

$\begin{array}{llllll}43 & 44 & 45 & 46 & 47 & 48\end{array}$ 
Consistently similar results were obtained when I determined progeny IBV production in HEK293-FIT-derived cells conditionally expressing WT TRIM56 or individual TRIM56 mutants (Figure 4-4). The $\sim 1-\log$ reduction in progeny virus yields ( $P$ values ranging from $<0.05$ to $<0.001)$ in + tet cells compared with -tet cells at 24 hpi with the $\mathrm{B} /$ Florida/4/06 virus $(\mathrm{MOI}=0.1)$ was abrogated only in cells harboring the 4693-750 mutant. Altogether, the domain-mapping experiments in HEK293-FIT- and MDBK-derived cells reached the same conclusion that the very $\mathrm{C}$-terminal tail region contains the sole prerequisite for TRIM56's antiviral activity against IAV and IBV. The shared TRIM56 determinant in restricting both genera of influenza viruses is consistent with the similar replication strategies of these orthomyxoviruses, and suggests that TRIM56 adopts the same antiviral mechanism against IAV and IBV.

\section{TRIM56's C-terminal Tail Is Sufficient to Curb Influenza Virus Infection}

Since our data showed that residues 693-750 at the C-terminal tail portion of TRIM56 is essential for the anti-influenza function, I asked if peptides derived from TRIM56's Cterminal region are sufficient to confer influenza virus restriction. To this end, I established HEK293-FIT-derived cell lines with tet-inducible expression of HA-tagged TRIM56 fragment comprising the C-terminal 135 (i.e., aa 621-755, referred to as CTT135) and 63 (i.e., aa 693-755, referred to as CTT63) residues, respectively (Figure 4-5A-B). When infected with $\mathrm{A} / \mathrm{PR} / 8 / 34$ virus $(\mathrm{MOI}=0.1)$, induction of CTT135 expression led to substantial decline in NP expression and progeny virus production, which mirrored the effect of induction of TRIM56 expression (Figure 4-5C). Induction of CTT63 produced a similar effect on NP expression (Figure 4-5D), suggesting the 63 residues at the very C-terminal end of TRIM56 contain the minimal determinant for the antiviral function of the full-length protein. The same could be said when I infected cells with B/Florida/4/06 virus (Figure 4-5E). The shared TRIM56 determinant governing the inhibitory effect on IAV and IBV implies that TRIM56 likely restricts both genera of influenza viruses via a similar mechanism. Thus, in subsequent experiments I utilized IAV to probe how TRIM56 executes its anti-influenza actions.

\section{TRIM56 Curtails Influenza Virus Propagation by Targeting Virus RNA Synthesis}

Following virus entry and uncoating steps, incoming influenza vRNPs are first transported into the nucleus, where viral RNA replication occurs $(15,20,21)$. Directed by a trimeric viral RNA polymerase complex in association with NP, vRNAs serve as the templates for synthesis of mRNAs and cRNAs, with the latter becoming the templates for synthesis of progeny vRNAs. Viral mRNAs are then transported to the cytoplasm for translation into nascent viral proteins that are required for $c$ RNA and vRNA synthesis (e.g., NP) and that serve structural and regulatory functions. At late stage of the infection, vRNAs are exported out of the nucleus for virion packaging and budding $(15,26)$. To determine at which step(s) of the influenza viral life cycle TRIM56 acts, I performed single-cycle growth analysis of IAV infection. MDBK-T56 cells with stable expression

of human TRIM56 and MDBK-Bsr cells (expressing the empty vector) were infected 


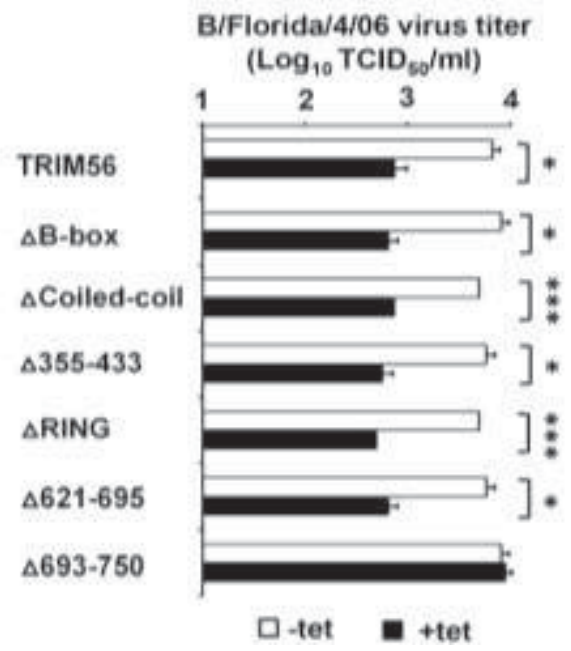

Figure 4-4. TRIM56's C-terminal tail is the only prerequisite for its anti-IBV function.

Progeny virus production in culture supernatants of 293-FIT-derived, T56 WT or individual mutant cells in the absence (-tet) or presence (+tet) of tet at 24 hpi with influenza $\mathrm{B} /$ Florida/4/06 virus $(\mathrm{MOI}=0.1)$. Single and triple asterisks indicate that statistical differences exist between -tet and +tet cells with a $P$ value of $<0.05$ and $<$ 0.001 , respectively. 


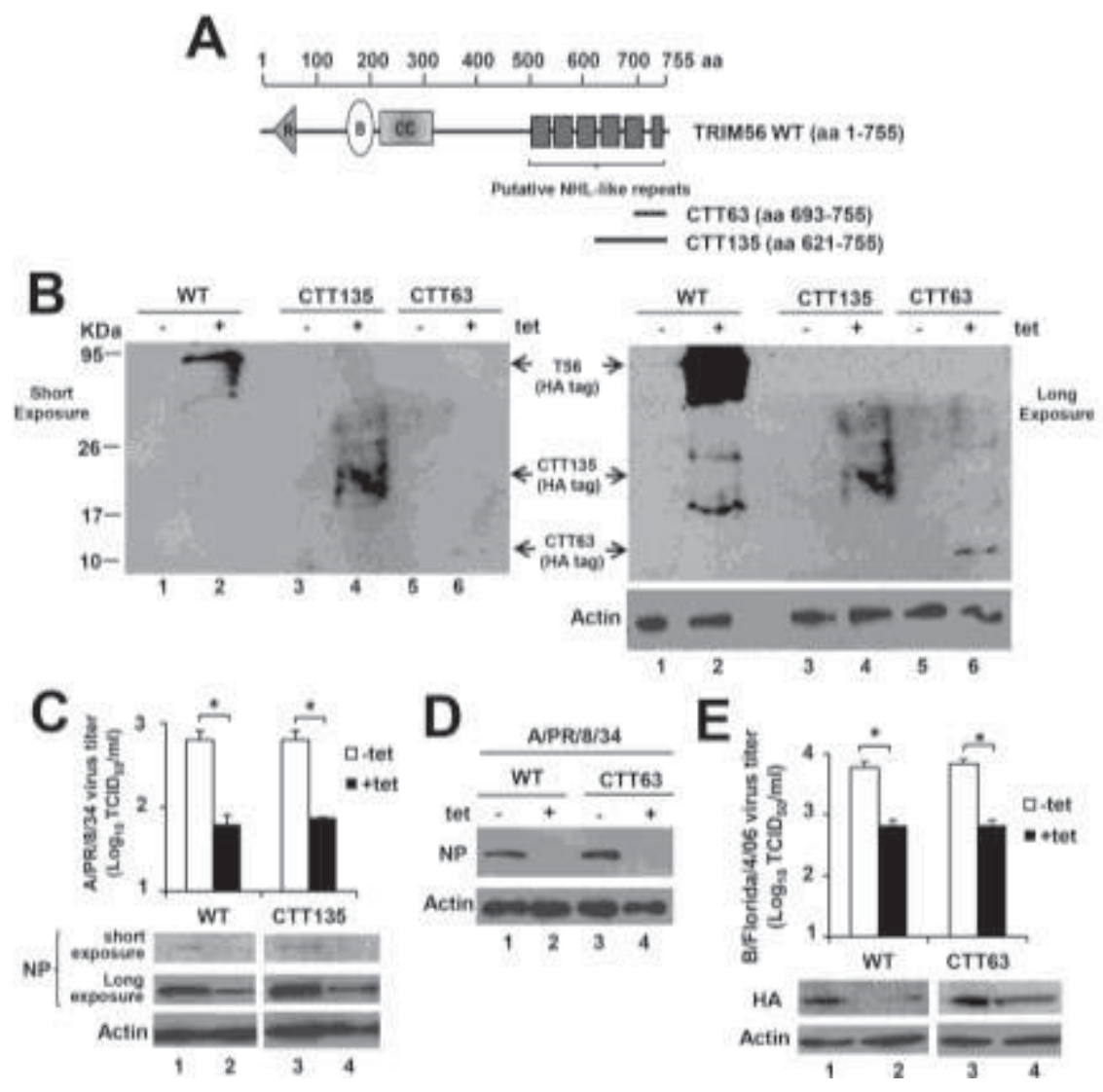

Figure 4-5. Expression of TRIM56's C-terminal tail alone is sufficient to curb infection by IAV or IBV.

(A) Schematic representation of putative NHL-like repeats in TRIM56's C-terminal portion and two peptides derived from TRIM56's C-terminal tail (CTT), i.e., TRIM56's C-terminal 135 residues (CTT135) and 63 residues (CTT63). (B) Immunoblotting HAtagged WT TRIM56, CTT135 and CTT63 (using anti-HA tag mAb (Invivogen)), and actin in HEK293-Flp-In T-REx-derived cells with tet-inducible expression of HA-tagged WT TRIM56 (293-FIT-T56-WT), CTT135 (293-FIT-T56-CTT135) or CTT63 (293-FITT56-CTT63) in the absence (-tet) or presence (+tet) of tet. (C) (Upper) Progeny virus titers in supernatants of 293-FIT-T56-WT or -CTT135 cells cultured with (+tet) or without tet (-tet) at 8 hpi with influenza $\mathrm{A} / \mathrm{PR} / 8 / 34$ virus $(\mathrm{MOI}=0.1)$. (Lower) Immunoblot analysis of NP (using anti-influenza A/WSN/33 (H1N1) NP mAb) and actin expression in the corresponding cell lysates. (D) Immunoblot analysis of NP (using antiinfluenza A/WSN/33 (H1N1) NP mAb) and actin expression in 293-FIT-T56-WT (left) or -CTT63 cells (right) cultured with (+tet) or without tet (-tet) at 8 hpi with influenza $\mathrm{A} / \mathrm{PR} / 8 / 34$ virus $(\mathrm{MOI}=0.1)$. (E) (Upper) Progeny virus titers in supernatants of 293FIT-T56-WT or -CTT63 cells cultured with (+tet) or without tet (-tet) at 24 hipi with influenza $\mathrm{B} /$ Florida/4/06 virus $(\mathrm{MOI}=0.1)$. (Lower) Immunoblot analysis of viral HA (using anti-influenza B/Hong Kong/8/73 HA pAb) and actin expression in the corresponding cell lysates. Single asterisk indicates that statistical difference exists between -tet and +tet cells with a $P$ value of $<0.05$. 
with influenza $\mathrm{A} / \mathrm{PR} / 8 / 34$ virus at an $\mathrm{MOI}$ of 3 , followed by a stringent wash in $0.9 \%$ $\mathrm{NaCl}(\mathrm{pH}=2.2)$ to remove extracellular virions that had not entered the cells. At 1, 4, 8 and 12 hpi, cells were washed extensively with PBS to eliminate extracellular viruses and viral RNAs before they were lysed for total RNA extraction and qPCR analysis of intracellular levels of vRNA, cRNA and mRNA of M segment. At the same time, yields of progeny viruses in cell-free culture supernatants at 8 and 12 hpi were determined by TCID $_{50}$ assay.

At 1 hpi, intracellular vRNA levels of $\mathrm{A} / \mathrm{PR} / 8 / 34$ virus were indistinguishable between MDBK-T56 and MDBK-Bsr cells (Figure 4-6A). In line with previous reports $(26,53,70,71)$, I found at this early time point IAV had already initiated mRNA transcription and cRNA synthesis in MDBK-Bsr cells. However, the abundance of cRNA (Figure 4-6B) and mRNA (Figure 4-6C) was 3-fold and 7-fold lower, respectively, in MDBK-T56 cells, suggesting that viral mRNA transcription and cRNA synthesis were hindered in TRIM56-overexpressing cells. At 4 hpi when viral RNA synthesis occurs at a high rate $(26,70,71)$, the abundance of vRNA rose by 5 -fold compared to that at $1 \mathrm{hpi}$ (Figure 4-6A), while cRNA (Figure 4-6B) and mRNA (Figure 4-6C) both climbed by $>3$ logs in MDBK-Bsr cells. In contrast, vRNA levels did not increase in MDBK-T56 cells at 4 hpi, suggesting onset of vRNA synthesis was delayed (Figure 4-6A). Although cRNA and mRNA levels increased compared to $1 \mathrm{hpi}$, they were 61 -fold and 88-fold lower, respectively, than those in MDBK-Bsr cells (Figure 4-6B-C). At 8 hpi when virus RNA synthesis had already finished $(26,71)$, I found that levels of all three RNA species reached plateau in MDBK-Bsr cells as well in MDBK-T56 cells (as compared to $12 \mathrm{hpi}$ ). Nonetheless, vRNA, cRNA, or mRNA levels remained 4-7 fold lower in MDBK-T56 cells at both 8 and 12 hpi (Figure 4-6A-C). These data show that there is a sustained influence on influenza virus vRNA, cRNA and mRNA levels throughout the life cycle in TRIM56-overexpressing cells.

In the same experiments (at 8 and $12 \mathrm{hpi}$ ), I found that progeny virus production in MDBK-T56 cells was significantly reduced compared with MDBK-Bsr cells, and the extent of reduction was proportional to the decrease in vRNA synthesis (data not shown), indicating that the effect on viral RNA synthesis is primarily responsible for TRIM56's anti-influenza action. Confirming that this observation is not cell-type specific, I obtained similar results when comparing time-course IAV RNA synthesis and progeny virus production between U2OS-T56 and U2OS-Bsr cells (data not shown). Therefore, it is confirmed that TRIM56 can curb influenza RNA synthesis in different cell types.

\section{The C-terminal Tail of TRIM56 Is Both Required and Sufficient for the Suppression of Influenza Virus RNA Synthesis}

To corroborate that TRIM56 impedes influenza virus RNA synthesis, I performed a luciferase reporter-based minigenome replication assay to measure IAV transcription and replication in HEK293-FIT-T56 cells with (+tet) and without (-tet) induction of HAtagged TRIM56. This system involves co-transfection of a total of six plasmids. Influenza RNA polymerase subunits (PB2, PB1, and PA) and NP derived from A/PR/8/34 virus are 

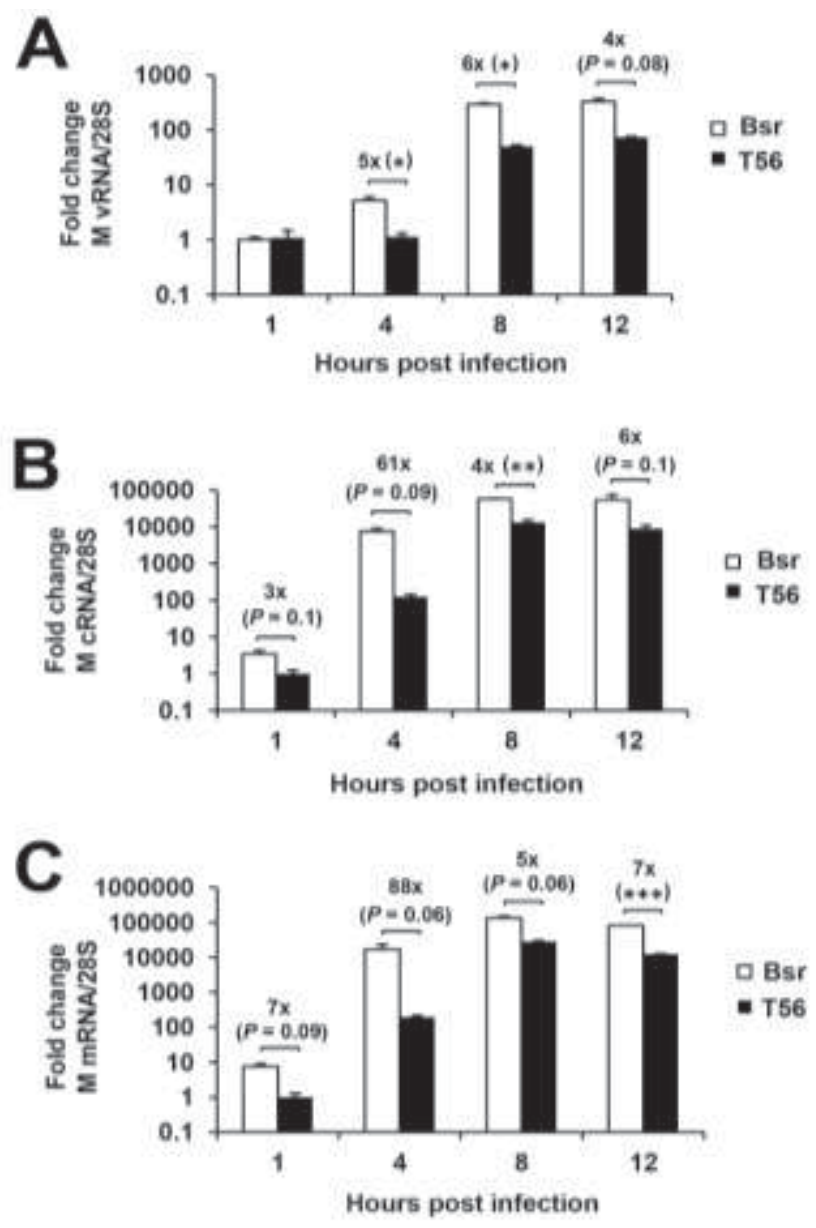

Figure 4-6. TRIM56 hinders IAV propagation by inhibiting intracellular virus RNA synthesis.

qPCR analysis of vRNA (A), cRNA (B) and mRNA (C) levels of matrix (M) gene segment of influenza $\mathrm{A} / \mathrm{PR} / 8 / 34$ virus in MDBK cells stably expressing a control vector (Bsr) or TRIM56 (T56) at 1, 4, 8 and $12 \mathrm{hpi}(\mathrm{MOI}=3)$. Single, double and triple asterisks indicate that statistical differences exist between Bsr and T56 cells with a $P$ value of $<0.05,<0.01$ and $<0.001$, respectively. 
expressed from individual plasmids under control of a Pol II promoter. In a fifth plasmid, the Pol I promoter controls transcription of the antisense strand of Firefly luciferase RNA flanked by the untranslated regions of $M$ segment vRNA (42). A sixth plasmid, pCMVRL, expresses Renilla luciferase from the CMV promoter, serving as an internal control for normalization of transfection efficiency. Since this assay was designed to mimic IAV transcription and replication steps, the Firefly/Renilla luc ratio (i.e., relative luciferase activity) is proportional to the level of influenza virus RNA synthesis $(42,53,54)$. I found that at both 24 and $48 \mathrm{~h}$ post transfection (hpt), relative luciferase activity was consistently 2-fold higher $(P<0.01)$ in -tet cells than in +tet cells (Figure 4-7A), corroborating the qPCR data on vRNA levels (Figure 4-6) and lending further support for the notion that TRIM56 inhibits influenza virus RNA synthesis. Additional minigenome replication assays in $\Delta 693-750$ mutant TRIM56-inducible cells and its parental HEK293-FIT (Ctrl) cells (Figure 4-7B, $48 \mathrm{hpt}$ ) showed that this C-terminal tail deletion mutant loses the ability to suppress influenza viral RNA synthesis and ruled out any effect due to tetracycline treatment. Further, expression of the CTT63 alone was sufficient to inhibit influenza viral RNA synthesis (Figure 4-7C), demonstrating that the C-terminal tail of TRIM56 dictates the antiviral restriction of influenza viruses by hindering the viral RNA synthesis step.

\section{TRIM56 Moves into the Nucleus during Influenza Infection}

Because TRIM56 is a cytosolic protein in resting cells (34), I wondered how this protein impacts influenza virus RNA synthesis, which takes place in the nucleus. To address this question I determined the subcellular localization of GFP-TRIM56 in transiently transfected HeLa cells prior to and after $\mathrm{A} / \mathrm{PR} / 8 / 34$ virus infection. As shown by the representative confocal fluorescence microscopy images (Figure 4-8A), while GFP-TRIM56 resided exclusively in the cytoplasm of mock-infected cells, GFP-TRIM56 fluorescence was detected in both cytoplasm and nucleus in IAV-infected cells at different time points post infection. Interestingly, the subcellular distribution pattern of HA-tagged TRIM56-CTT63 mirrored that of full-length, HA-tagged TRIM56

(Figure 4-8B). While there was no appreciable nuclear immunostaining of CTT63 in uninfected cells, a fraction of CTT63 was reproducibly found in the nucleus after IAV infection. The presence of TRIM56 and TRIM56-CTT63 in the nucleus of IAV-infected cells is consistent with their ability to impede influenza viral RNA synthesis.

\section{Discussion}

In this study, I have shown that TRIM56 is an intrinsic host restriction factor of influenza A and B viruses (Figure 4-1 and 4-2), expanding the antiviral spectrum of this TRIM to include the Orthomyxoviridae family of segmented, negative-strand RNA viruses. Ectopic expression of TRIM56 impeded propagation of these medically important respiratory pathogens (Figure 4-1), while silencing of endogenous TRIM56 expression had the opposite effect (Figure 4-2). Importantly, these findings were reproduced in multiple cell types, irrespective of the expression system. Moreover, the 

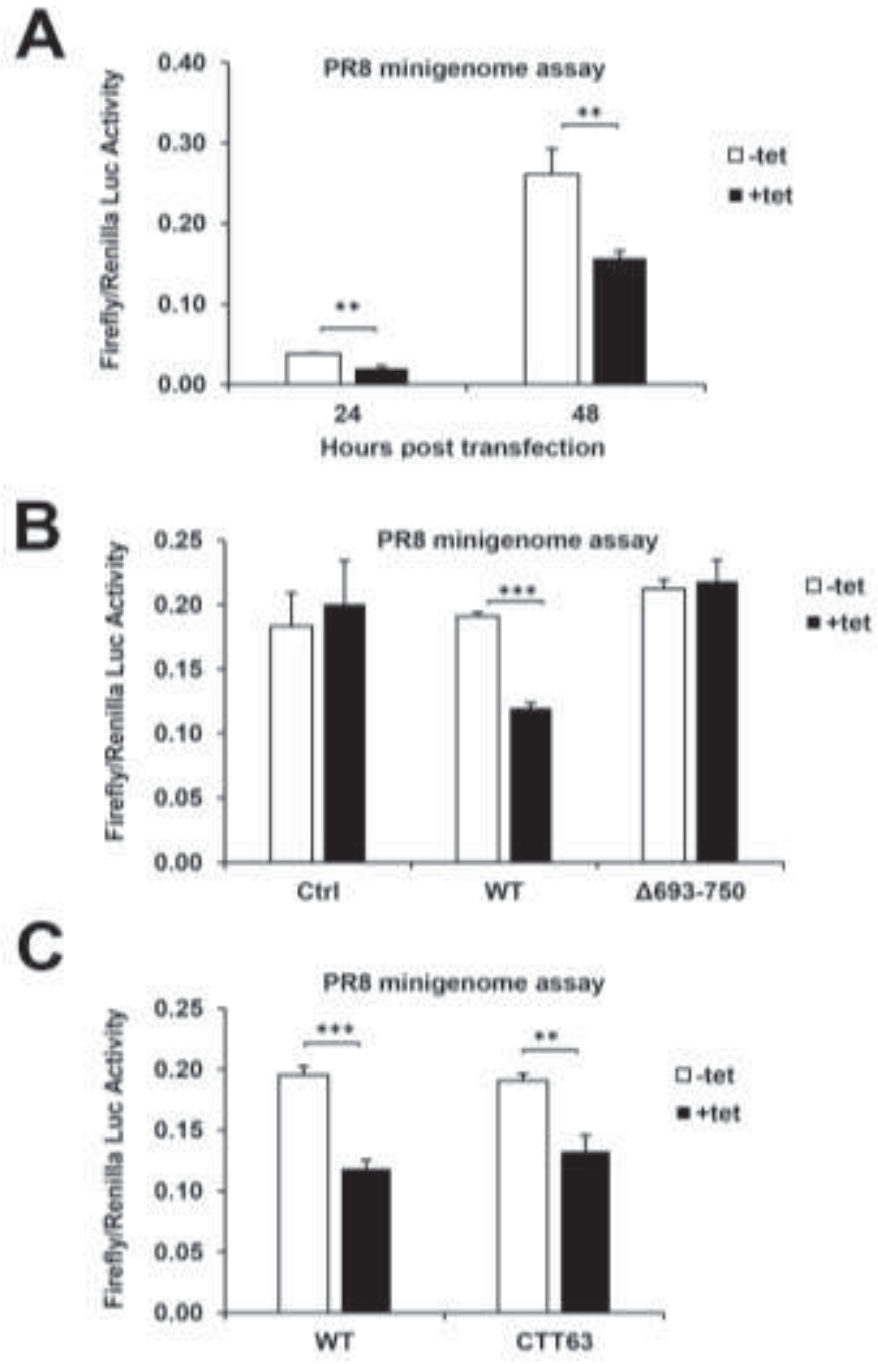

Figure 4-7. The C-terminal tail portion is required for TRIM56 suppression of influenza virus RNA synthesis.

(A) Luciferase (luc) reporter-based minigenome replication assay of IAV RNA synthesis was performed in 293-FIT-T56 cells cultured in the presence (+tet) or absence (-tet) of tet at 24 and $48 \mathrm{~h}$ post co-transfection (hpt) with polymerase II-driven $\mathrm{pHW}$ plasmids expressing PB2, PB1, PA and NP, a polymerase I-driven luc reporter plasmid pHY-Luci, and a CMV promoter-driven plasmid expressing the Renilla luc (pRL-CMV, serving as an internal control for normalization of transfection efficiency). (B) Luciferase reporterbased minigenome replication assay of IAV RNA synthesis in parental 293-FIT (Ctrl),

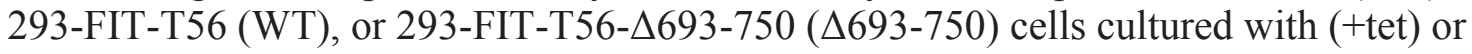
without tet (-tet) at $48 \mathrm{hpt}$. (C) Luciferase reporter-based minigenome replication assay of IAV RNA synthesis in 293-FIT-T56 (WT) or 293-FIT-T56-CTT63 (CTT63) cells cultured with (+tet) or without tet (-tet) at $48 \mathrm{hpt}$. Double and triple asterisks indicate that statistical differences exist between -tet and +tet cells with a $P$ value of $<0.01$ and $<$ 0.001 , respectively. 
Figure 4-8. TRIM56 and TRIM56-CTT63 move into the nucleus after IAV infection.

(A) HeLa cells transiently transfected with an N-terminal GFP-tagged TRIM56 (GFPT56) plasmid was mock-infected or infected with influenza A/PR/8/34 virus for 4,8 and $16 \mathrm{~h}(\mathrm{MOI}=3)$, followed by immunostaining of NP (using anti-influenza A/WSN/33 (H1N1) NP mAb). The subcellular localizations of GFP-T56 (green fluorescence) and NP (red fluorescence) were examined by confocal microscopy. Nuclei were counterstained blue with DAPI (4',6-diamidino-2-phenylindole). A scale bar represents $10 \mu \mathrm{m}$. (B) 293FIT-T56 or 293-FIT-T56-CTT63 cells were cultured in the presence of tet to induce expression of HA-tagged, TRIM56 (WT) or TRIM56-CTT63, followed by mock infection or infection with influenza $\mathrm{A} / \mathrm{PR} / 8 / 34$ virus for $16 \mathrm{~h}(\mathrm{MOI}=3)$. HA-tagged TRIM56 and TRIM56-CTT63 were immunostained with an anti-HA tag mAb (Invivogen), and IAV matrix (M) proteins with a goat anti-matrix antiserum. The subcellular localization of HA-tagged WT TRIM56 and TRIM56-CTT63 (green fluorescence) and $\mathrm{M}$ (red fluorescence) proteins was examined by confocal microscopy. Nuclei were counterstained blue with DAPI. Scale bars represent $5 \mu \mathrm{m}$. 


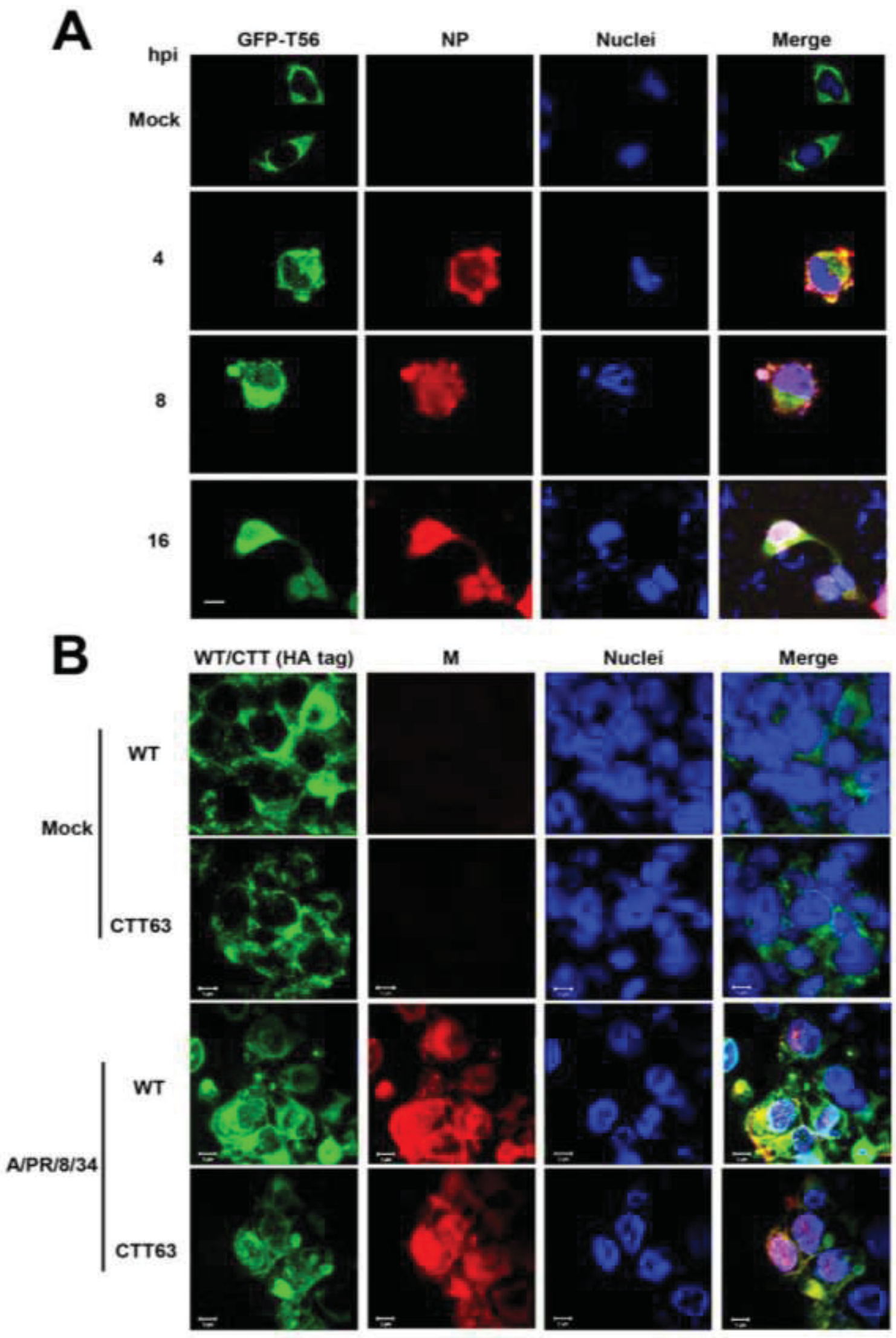


antiviral effect was specific for influenza viruses since TRIM56 did not inhibit $\mathrm{SeV}$ or hMPV (Figure 4-1), paramyxoviruses that cause respiratory infections in mice and humans, respectively. To our knowledge, this is the first study that demonstrates TRIM56 targets negative-strand RNA viruses for inhibition.

TRIM56 positively regulates innate antiviral signaling through TLR3- and STINGdependent pathways. Specifically, TRIM56 forms a complex with TRIF, the TLR3 adaptor, via its $\mathrm{C}$-terminal region, promoting IRF3 activation and subsequent IFN response in ways independent of its E3 ligase activity (39). In contrast, the mechanism underlying the augmentation of STING-dependent IFN-induction pathway is distinct. In this case TRIM56 associates with STING and acts as an E3 ligase that promotes Lys63linked ubiquitination of STING, thereby enhancing cytosolic DNA-induced IFN production (58). In previous studies $(34,35)$ we have shown that a heightened IFN response is not responsible for TRIM56-mediated inhibition of four positive-strand RNA viruses, BVDV, YFV, DENV2 or HCoV-OC43. This may be also the case for the novel anti-influenza activity of TRIM56 described in this study. First, I found no evidence of IFN induction or upregulation of ISGs in all cell types examined in this study following $\operatorname{IAV}(\mathrm{A} / \mathrm{PR} / 8 / 34)$ infection, regardless of TRIM56 expression status (data not shown). This was not surprising given that IAV encodes multiple IFN antagonists, with NS1 being the most potent and characterized one $(25,72)$. Second, in cell culture innate antiviral response to IAV is primarily mediated by RIG-I while TLR3 plays a more proinflammatory role $(22,73)$. However, TRIM56 does not contribute to or regulate RIGI/MDA5 signaling (39). Third, although STING-dependent pathway significantly contributes to host defense against DNA viruses and bacterial infections, direct evidence for this pathway in fending off RNA virus infections is lacking $(58,62)$. Also, it is worth noting that I demonstrated the anti-influenza effect of TRIM56 in cell lines derived from HEK293 cells, which have undetectable STING protein expression (35).

Of $>70$ TRIMs, two (TRIM22 and TRIM32) have been shown to inhibit influenza virus. Both critically hinge on the E3 ligase activity to operate by promoting viral protein degradation, with TRIM22 targeting NP (33) while TRIM32 acting on PB1 (74). However, TRIM56's anti-influenza mechanism is distinct, which appears to be completely uncoupled from the E3 ligase activity and does not require the RING, B-box, or coiled-coil domains, either (Figure 4-3 and 4-4). This E3 ligase-independent mechanism is also in stark contrast to what is known about the antiviral activities of TRIM56 against flaviviruses and HCoV-OC43 (35). Remarkably, a TRIM56 fragment of as few as 63 residues at the $\mathrm{C}$-terminus of the protein recapitulated the anti-influenza effect imposed by full-length TRIM56 (Figure 4-5), indicating that a fully functional motif mediating influenza virus restriction is encoded by this small portion of the protein. Given its small footprint, this anti-influenza determinant may be amenable to therapeutic manipulations. Additional, refined mapping and codon optimization may lead to a TRIM56-derived peptide/small molecule that has broad activity against different influenza viruses, since both IAV and IBV are susceptible to TRIM56's action.

Our time-course viral RNA quantification data (Figure 4-6) and influenza minigenome replication assay (Figure 4-7) show that TRIM56 specifically inhibits 
influenza viral RNA synthesis by its C-terminal tail portion. Consistent with the fact that influenza viral RNA synthesis occurs in the nucleus, I found that a fraction of TRIM56 localized into the nucleus in IAV-infected cells, in contrast with the exclusively cytosolic distribution of TRIM56 in uninfected cells (Figure 4-8). However, it is unknown whether the nuclear translocation of TRIM56 following influenza virus infection is an active, coordinate cellular process to combat the virus, or that TRIM56 is passively imported into or retained in the nucleus while it is associated with viral RNPs or host factors recruited to the RNPs. Further studies will be required to clarify this. Interestingly, TRIM32, which is another IAV-inhibitory TRIM, translocates to the nucleus following IAV infection as a result of its association with and retention by viral PB1 protein (74). In addition, a search using the NCBI conserved domain database showed that there exists sequence homology between the C-terminal residues 521-748 of TRIM56 and the NHL repeats of several well-characterized TRIM-NHL proteins (TRIM2, TRIM3, TRIM32 and TRIM71) (Figure 4-9) (75,76). Of note, some of these TRIM-NHL proteins have the ability to regulate RNA abundance and metabolism $(75,76)$, raising the possibility that TRIM56 might bear a similar function. Further investigation is still required to elucidate precisely how TRIM56 hinders influenza virus RNA synthesis, knowledge of which will provide new insights into factors and molecular interactions that control this vital step in influenza virus life cycle. 
Figure 4-9. Sequence alignment of the C-terminal portion of TRIM56 with the NHL repeats of well-characterized TRIM-NHL proteins.

The C-terminal amino acid sequence of TRIM56 (GenBank ID: NP_112223) was aligned and compared with the NHL repeat sequences of the following TRIM-NHL proteins, including NCL-1 (Swiss-Prot P34611), LIN-41 (Swiss-Prot Q9U489), KIAA0517 (GenBank ID: BAA25443), Brat (GenBank AAF53771), TRIM3 (GenBank ID: NP_001234936), TRIM2 (GenBank ID: NP_056086) and TRIM71 (GenBank ID: NP_001034200). The numbering of the repeats in each protein was indicated as blades IVI. Numbers show the beginning (left) and ending (right) positions of each repeat of the proteins. Resides that are $>50 \%$ conserved are highlighted by shade in color. Consensus residues at each conserved amino acid positions across different blades are shown at the bottom in the consensus sequence, in which " $h$ " represents the hydrophobic residues isoleucine, leucine and valine. Asterisks denote the stop codon. 


\section{Blades}

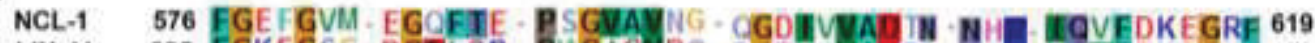

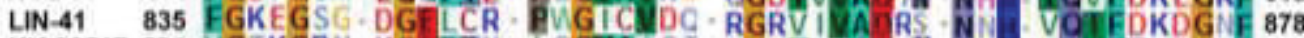
KIAA0517 524 VGTKGRN-KGIFIN- LCGVAAS T-NGKLLTA SN-NQC. VOO FSIDGCF 567

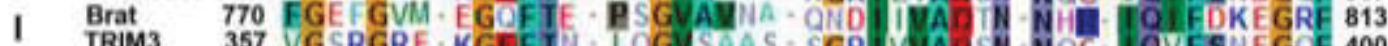
357 VGSRGRE - KGFF IN - LOGUSAAS - SGR IVUAISN-NOC. I OVFSNEGCF 400

TRIM2 503 VGTKGRN-KGEFIN-LGGVAAST-NGK LTALSN.NOC. VOTIF SIDGCF 546 TRIM71 596 FGSEGDS-DGKLCR - PVGUSNDK-EGY INAERS-Nit TOVFKPCGAE 639 TRIM56 497 FPTRIIPG - DKR SPR - T TGLCPFG - PRE I UAIEO-NRA- LKRFSLNGDY 540

NCL-1 623 FGECGKD -GOELY - PURUAPNRTTGREVNTERSPTHO- IOVYNOYGOF 668

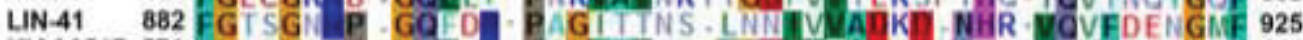

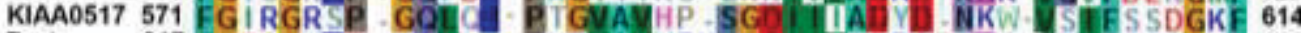

II Brat 817 FGECGKD . SQtLY. PNR VAVVRNSGOIVTERSPTHO-TO YNOYGOF $\mathbf{8 6 2}$

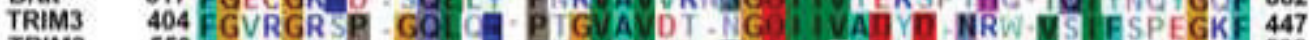
TRIM2 550 FGIRGRS. GQI

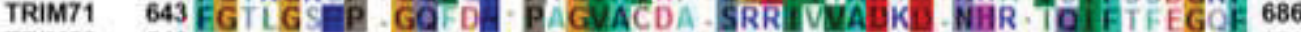
TRIM56 542 ............. GTVPV. PEGCSPC SVRALOSAVAFSASAR. LYLTNPIGEV 579

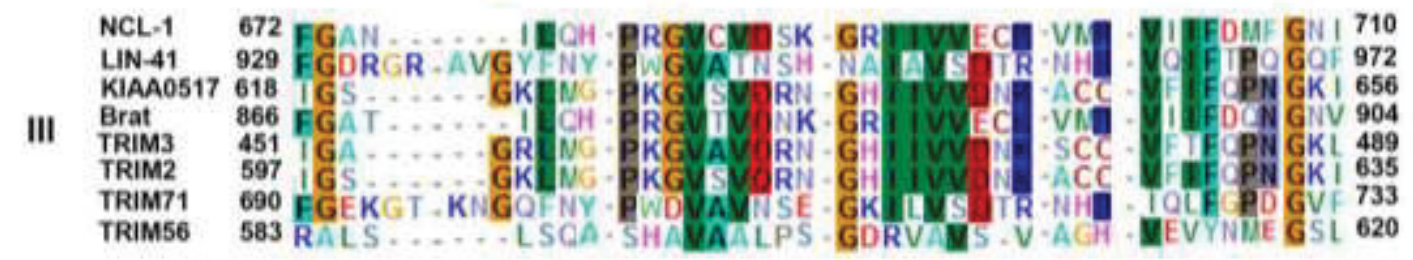

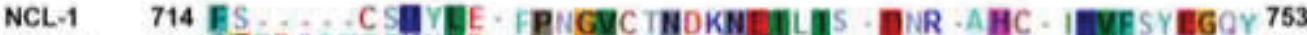

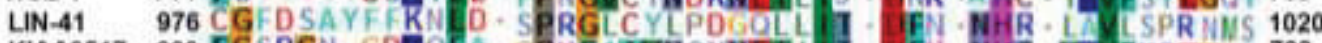

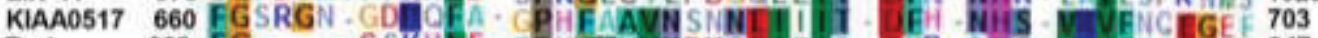
Brat $\quad 908$ FG........ CSKHLE. FPNGYVNDKO

TRIM3 493 EGCRGA TDPHEA

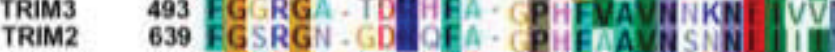

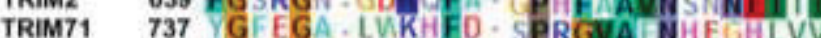

TRIM56 624 ETPGGK-ASAGER-ALVFTTTSPQGHFVGS

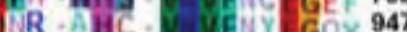
FH. NiHS. VUVSADGEF 536 FH. WHS. UNGICGE 682

NCL-1 757 IG ....GE-GVTNY-PIGVGINS - LGEVVNARNH .MNENLTVFSODGIM 797

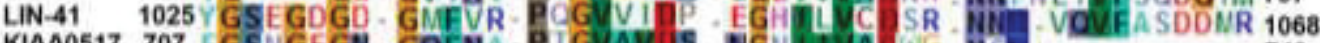
KIAA0517 707 FGSNGEGN-GOFNA.PIG ANISS NGIIIUA WG NS .

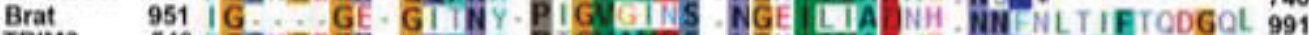

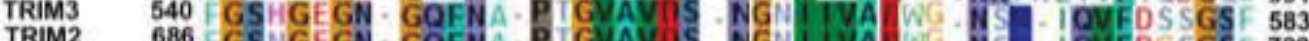

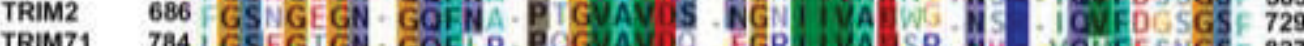

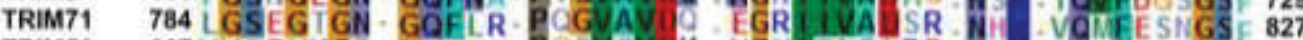
TRIM56 667 VVGEYKGP. GLHGCORGSUSVIK . KGYIFLTLRE. VNK. VVITDPKGSL 711

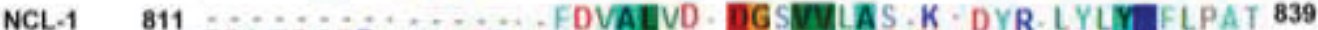

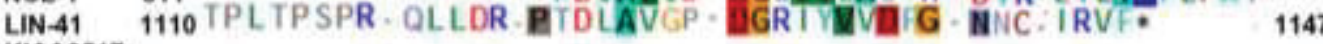
KIAA0517

VI Brat

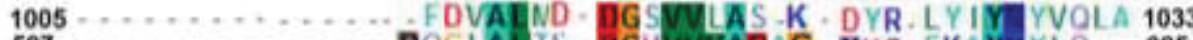
TRIM3 $597 \ldots \ldots \ldots \ldots \ldots \ldots$ PQGLAI TS - DGHVVVADAG - NHC. FKAY YLQ. 625

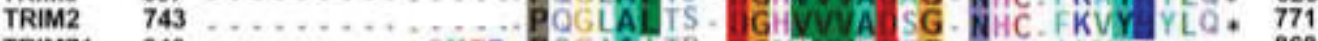

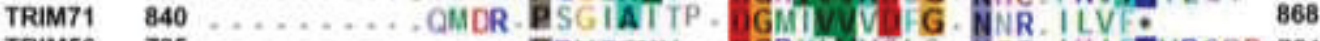

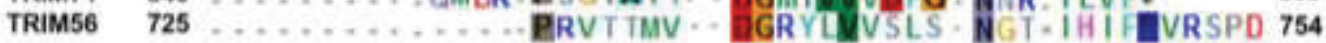

Consensus FG.,G...-GQL..-P.GVAV.S-.G.IhVAD.,-NHR-hQVF...G. F 


\section{CHAPTER 5. MOLECULAR DETERMINANTS GOVERNING TRIM56- MEDIATED POSITIVE REGULATION OF THE TLR3 ANTIVIRAL SIGNALING PATHWAY}

\section{Introduction}

In response to viral invasion, the innate immune system constitutes a front line of host defense armed with multi-layered mechanisms. Of these, pattern recognition receptors (PRR), such as Toll-like receptors (TLRs) and retinoic-inducible gene-I (RIGI)-like receptors, play important roles in recognizing viral nucleic acids as a major class of viral pathogen-associated molecular patterns. That recognition triggers intracellular signaling pathways that culminate in activation of interferon regulatory factor 3 (IRF3) and NF- $\mathrm{KB}(1,2)$. These two transcription factors play pivotal parts in production of interferons (IFNs) and pro-inflammatory cytokines/chemokines. Type I IFNs (IFN- $\beta$ and IFN- $\alpha$ ) further induce hundreds of IFN-stimulated genes (ISGs) to establish an antiviral state restricting viral propagation and spread (1-4).

Over the past decade, TRIM family members as intracellular restriction factors are increasingly recognized as active players in antiviral innate immunity (28-31).

Structurally, TRIMs possess a highly conserved arrangement of domains in the Nterminal RBCC motif (i.e., RING, B-box and coiled-coil domains), but their C-terminal tails vary. In general, RING domains confer TRIMs with E3 ubiquitin (Ub) ligase activity. B-box domains are zinc-binding motifs while coiled-coil domains facilitate selfassociation and oligomerization and act as scaffolds for recruitment and formation of multi-protein complexes $(30,31)$. Much attention has been paid to delineate direct antiviral roles of the TRIMs contributing to host restriction of specific types of viruses by targeting various steps in the viral life cycle. As an example, TRIM22 promotes polyubiquitination of the NP of influenza A virus (IAV) and targets it for proteasomedependent degradation (33). Work from our group has revealed that TRIM56 is a versatile antiviral host factor that restricts distinct RNA viruses including human coronavirus-OC43 and several members of the family Flaviviridae, via overlapping and distinct molecular determinants, $(34,35)$. I recently found that TRIM56 also restricts influenza A and B viruses by suppressing viral RNA synthesis via its C-terminal tail, independent of the E3 ligase activity and other portions of TRIM56 (36).

Recently, the "indirect" antiviral roles of various TRIMs, i.e., through positively regulating innate antiviral immune signaling pathways and augmenting IFN responses, have begun to be appreciated. Versteeg et. al reported that approximately half of the 75 TRIMs have the ability to act at different junctures in innate immune signaling pathways, thereby promoting antiviral responses (37). In this group of TRIMs, TRIM25 depends upon its E3 ligase activity to ubiquitinate and activate RIG-I, thereby potentiating the RIG-I-mediated signaling pathway and downstream IFN induction $(38,77)$. As another example, TRIM56 heightens cytosolic dsDNA-stimulated IFN production by interacting with and ubiquitinating stimulator of IFN genes (STING), an endoplasmic reticulum residing adaptor protein for innate immune signaling (58). Interestingly, our lab has 
shown that TRIM56 also promotes viral activation of IRF3 via the TLR3 pathway. In this case TRIM56 operates independent of its E3 ligase activity or the entire RING domain but, instead, involves an association with the TLR3 adaptor protein, i.e., TIR-domaincontaining adapter-inducing IFN- $\beta$ (TRIF), via its C-terminal region (39). However, the precise mechanism by which TRIM56 positively modulates TLR3 signaling remains elusive. Also unclear is the detailed TRIM56 determinants governing this property. In this study, I made efforts to delineate roles of the coiled-coil domain and several putative phosphorylation sites residing in the C-terminal region of TRIM56 in the up-regulation of TLR3-dependent antiviral immunity.

\section{Results}

\section{TRIM56 Promotes TLR3-mediated, IRF3- or NF-кB-dependent Signaling Pathway via Forming a Complex with TRIF}

We have previously reported that human TRIM56 positively regulates TLR3mediated IRF3-dependent signaling pathway (39). Bioinformatic analysis suggests there is $81 \%$ amino acid (aa) identity between human (here referred to as hTRIM56 or hT56) and murine TRIM56 (here referred to as mTRIM56 or mT56), though the latter is 22-aa shorter than the former. I wondered if mTRIM56 would have a similar property as hTRIM56 for regulating TLR3 signaling. To this end, dsRNA-induced IFN- $\beta$ promoter activity with or without ectopic expression of mTRIM56 was determined (Figure 5-1A). Compared with the control vector, ectopic expression of mTRIM56 enhanced poly-I:Cinduced IFN- $\beta$ promoter activity by 5.2 folds $(P<0.00001)$, while at the same time overexpression of hTRIM56 augmented IFN- $\beta$ promoter activity by 2.6 folds $(P<0.001$, Figure 5-1A, right). This data suggest that mTRIM56 is a stronger, positive regulator of TLR3 signaling than hTRIM56.

Previously we found TRIM56 acts at the level of TRIF to modulate the TLR3 signaling pathway. I reasoned that TRIM56 may also influence the activation of NF- $\kappa \mathrm{B}$, another transcription factor downstream of TRIF in the TLR3 signaling pathway. As expected, TRIM56 overexpression heightened NF- $\kappa \mathrm{B}$-dependent PRDII promoter activation following the TLR3 engagement. However, it had no effect on PRDII promoter activation induced by TNF- $\alpha$ - or IL-1 $\beta$ (Figure 5-1B). Thus, TRIM56 specifically positively regulates NF- $\mathrm{KB}$ activation through TLR3 signaling pathway. This evidence also confirmed that TRIM56 specifically acts on the TLR3 pathway prior to the bifurcation of NF- $\mathrm{BB}$ and IRF3. Consistent with this, I detected endogenous TRIM56 and TRIF proteins associated with each other early (0.5 - 2 hours) following exposure to extracellular poly-I:C, when there was no up-regulation of ISG56 (Figure 5-1C). Altogether, the aforementioned experiments corroborated that TRIM56 is a positive regulator of TLR3-mediated IRF3- and NF- $\kappa B$-dependent signaling via its interaction with TRIF. 
Figure 5-1. TRIM56 positively regulates TLR3-mediated IRF3- or NF-кBdependent signaling pathway by forming a complex with TRIF.

(A) (Left) Immunoblot analysis of the expression of vectors encoding C-terminally V5tagged mouse TRIM56 (mT56-V5) and human TRIM56 (hT56-V5) (using mouse antiV5 monoclonal antibody (mAb)) in human embryo kidney (HEK) 293 cells. Actin serves as loading control. (Right) HEK293-TLR3 cells which ectopically express TLR3 were co-transfected with a plasmid encoding hT56 or mT56, or the corresponding empty vector (vector), as well as internal control plasmid pRL-TK and reporter plasmid IFN- $\beta$ luc, followed by mock treatment or treatment with poly-I:C (pIC) for $8 \mathrm{~h}$. Dual luciferase (luc) reporter assay was then performed to measure the promoter activation. (B) (Left) Immunoblot analysis of the ectopic expression of N-terminally Flag-HA-tagged TRIM56 (FH-T56) (using mouse anti-HA mAb) in HEK293-TLR3-YFP (293-T3Y)-derived cells over-expressing FH-TRIM56 (293-T3Y-FH-T56). HEK293-T3Y-FH-T56 and its parental 293-T3Y cells were co-transfected with internal control plasmid pRL-TK and reporter plasmid IFN- $\beta$-luc (middle), or NF- $\kappa$ B responsive reporter plasmid PRDII-luc (right), followed by mock treatment or treatment with TNF- $\alpha$, IL-1 $\beta$ or poly-I:C for $8 \mathrm{~h}$. Dual luc reporter assay was then performed to measure the corresponding promoter activation. (C) Co-immunoprecipitation (co-IP) analysis of the interaction between endogenous TRIM56 with endogenous TRIF in HeLa cells at the indicated time points post poly-I:C treatment. Cell lysates were immunoprecipitated (IP) with anti-TRIM56 polyclonal $\mathrm{Ab}$ (pAb), followed by immunoblotting (IB) with anti-TRIF or anti-TRIM56. The upper blot showed expression of TRIM, TRIF and IFN-stimulated gene 56 in cell lysates. Single asterisk indicates that statistical differences exist between mock- and polyI:C-treated cells with a $P$ value of $<0.05$. N.S., not statistically significant. 
A

IB: actin
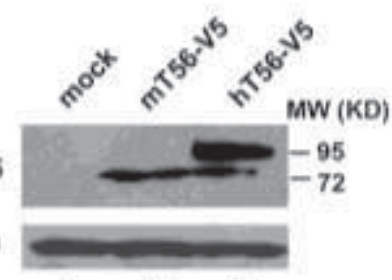

1

2

3
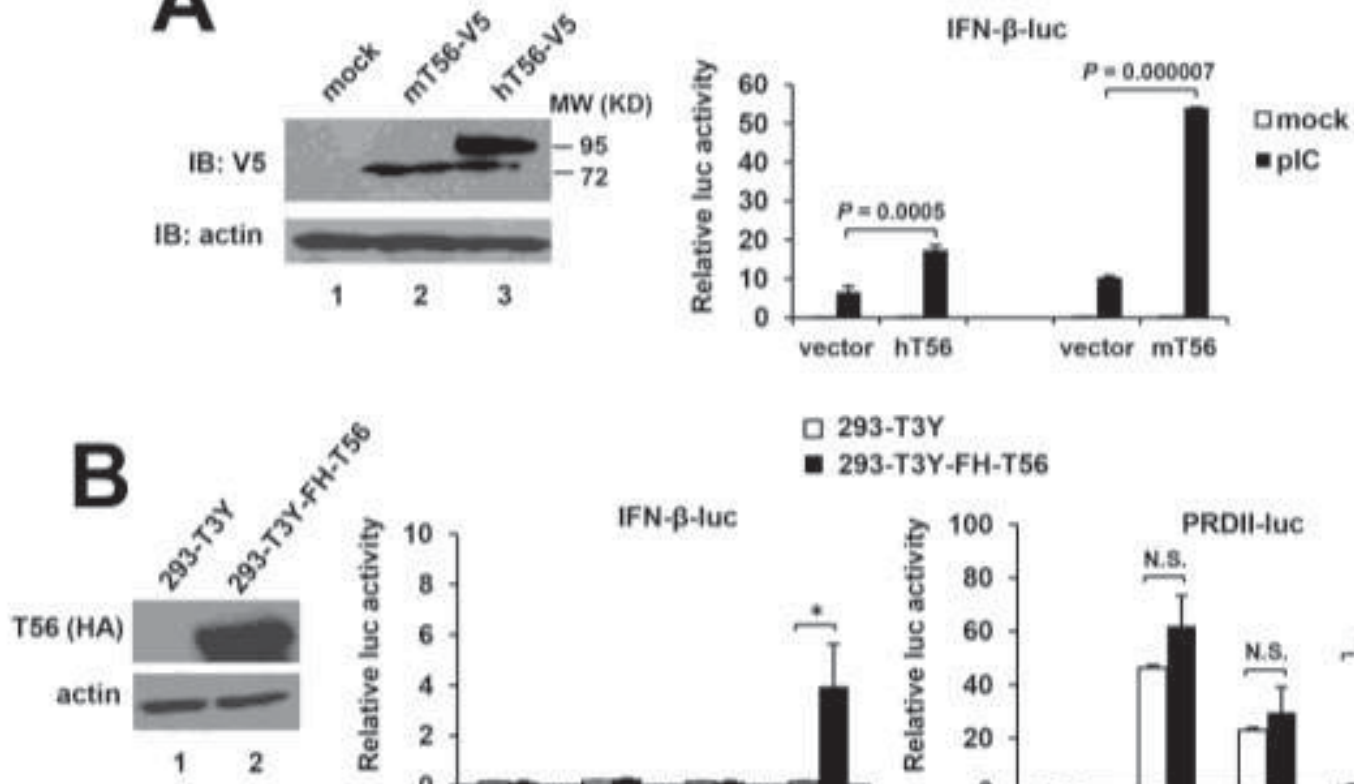

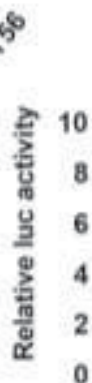

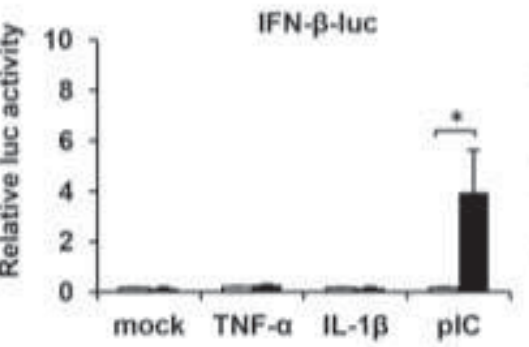

- 293-T3Y-FH-T56

293-T3Y

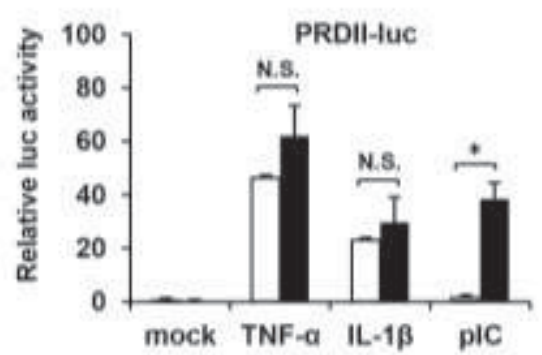

\begin{tabular}{llllll} 
Hours post plC treatment \\
\hline 0 & 0.5 & 1 & 2 & 4 & 9
\end{tabular}
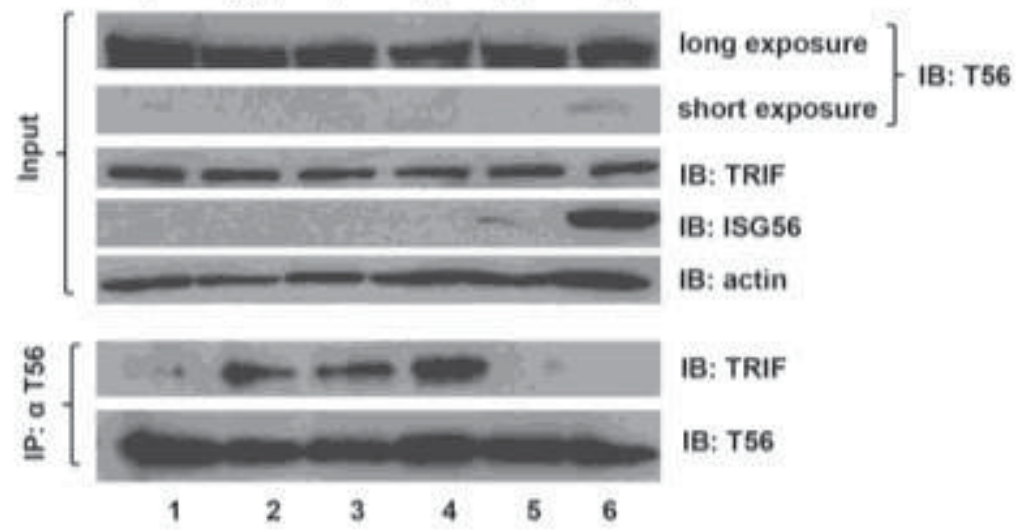

IB: TRIF

IB: T56 
The Coiled-coil Domain and Residues 431-610 of TRIM56, but Not B-box or Residues 355-433, Are Critical for Enhancing TLR3-mediated IRF3- or NF-кBdependent Signaling

We have previously demonstrated that TRIM56's C-terminal end (aa 621-750) is critical for its positive regulation of TLR3-mediated IRF3-dependent signaling, while the E3 ligase activity and the entire RING domain are dispensable (39). However, the contribution of other portions of TRIM56 to this biological process is unknown. To better understand this, I measured poly-I:C-induced IFN- $\beta$ promoter activities after transient expression of WT and various mutant TRIM56-V5 (Figure 5-2A). The mutants studied in this assay included those lacking the RING domain (referred to as $\triangle$ RING), B-box domain (referred to as $\Delta \mathrm{B}$-box), and all ( $\Delta$ Coiled-coil) or part ( $\Delta 211-284$ and $\Delta 290-353$ ) of the coiled-coil domain, respectively, and mutants bearing different deletions in the Cterminal portion $-\Delta 355-433$ (lacking aa 355-433), $\Delta 431-519$ (lacking aa 431-519), $\Delta 515-610$ (lacking aa 515-610), $\Delta 693-750$ (lacking aa 693-750) and $\Delta 369-742$ (lacking aa 369-742). In addition, we examined the $\Delta 2-363$ mutant, which lacked residues 2-363 thus losing the entire RBCC motif and a stretch of downstream sequences. The expression levels of these V5-tagged TRIM56 mutants were comparable to that of WT TRIM56 (data not shown; 34). Confirming our previous observation (39), the $\triangle$ RING mutant retained the ability to promote TLR3 signaling, acting similarly to WT TRIM56 whereas deletions in the C-terminal end ( $\Delta 693-750)$ abolished TRIM56's ability to promote TLR3 signaling $(P<0.01$, Figure 5-2A). Consistent with the latter result, the $\triangle 369-742$ mutant bearing a larger deletion in the $\mathrm{C}$-terminal region (i.e., lacking nearly the C-terminal half of TRIM56) was also devoid of such capability (Fig. 5-2A). Interestingly, I found that the C-terminal portion of TRIM56 required for positive regulation of TLR3 signaling also included aa 431-610, since ectopic expression of $\Delta 431$ 519 and $\Delta 515-610$, but not $\Delta 355-433$, failed to augment TLR3-mediated IFN- $\beta$ promoter activation, as compared with WT TRIM56 $(P<0.01)$, respectively. As to the N-terminal domains, $\Delta \mathrm{B}$-box was found to maintain the ability to enhance poly-I:C-induced IFN- $\beta$ promoter activity. In contrast, $\Delta 2-363$ lacking the RBCC motif was unable to do so, suggesting a requirement for the coiled-coil domain. Confirming this notion, deletions of the entire coiled-coil ( $\Delta$ Coiled-coil) or part of this domain $(\Delta 211-284$ and $\Delta 290-353)$ abolished the function of TRIM56 in heightening TLR3 signaling $(P<0.01$ to $<0.001)$. In aggregate, these data demonstrated that the integrity of the $\mathrm{C}$-terminal portion spanning aa 431-750 is critical for the ability of TRIM56 to promote TLR3 signaling, as is the coiled-coil domain. However, the B-box is dispensable. These data were confirmed by examining TLR3-mediated activation of IFN- $\beta$ or IL-29 (IFN- $\lambda 1$ ) promoters in 293FIT-T56 WT/Mut cells with tet-inducible expression of HA-tagged WT/mut TRIM56 (Figure 5-2B) .

TRIM56 can self-associate to form dimers, and the coiled-coil domain has been reported to mediate formation of anti-parallel dimers for TRIM molecules $(34,66)$. To ascertain that the $\Delta \mathrm{B}$-box and $\Delta 355-433$ TRIM56 mutants are indeed capable of promoting TLR3 signaling, one has to exclude the possibility that functions of these mutants are dependent upon their pairing with endogenous, WT TRIM56. Our previous (36) and current qPCR data (Figure 5-2C, bottom) suggest that the mRNA level of the 
Figure 5-2. TRIM56's coiled-coil domain and residues 431-610, but not the B-box or residues 355-433, are required for its augmentation of TLR3-mediated IRF3dependent signaling pathway.

(A) (Left) Schematic representation of wild-type (WT) and various TRIM56 mutants to be investigated in this study. R, B and CC represent RING, B-box and Coiled-coil domains, respectively. (Right) HEK293-TLR3 cells were co-transfected with an empty vector or vectors encoding WT and various mutant TRIM56-V5, as well as internal control plasmid pRL-TK and reporter plasmid IFN- $\beta$-luc, followed by mock- or poly-I:Ctreatment (for $8 \mathrm{~h}$ ) and dual luc reporter assay. (B) Tetracycline (tet)-inducible HEK293Flp-In-T Rex (FIT)-derived cells conditionally expressing N-terminally HA-tagged, WT and mutant (mut) TRIM56 (HEK293-FIT-T56 WT/Mut) in the absence (-tet) or presence (+tet) of tet, were co-transfected with TLR3-encoding vector, $\mathrm{pRL}$-TK and reporter plasmid IFN- $\beta$-luc (upper part) or IL-29-luc (lower part), followed by mock- or poly-I:Ctreatment (for $8 \mathrm{~h}$ ) and dual luc reporter assay. (C) (Top) Immunoblot analysis of expression levels of endogenous and exogenously overexpressed (HA-tagged) TRIM56 (using anti-TRIM56) in HEK293-FIT-T56 cells with (+tet) or without (-tet) tet treatment for $48 \mathrm{~h}$. qRT-PCR was carried out to measure the abundance of endogenous TRIM56 transcript (middle, using primers amplifying the 3' UTR of TRIM56 mRNA), total TRIM56 transcript including the endogenous and exogenously overexpressed TRIM56 mRNAs (bottom, using primers amplifying the coding region of TRIM56 mRNA) in 293FIT-T56 cells cultured with (+tet) or without (-tet) tet, and transfected with a negative control siRNA (Ctrl) or siRNA targeting 3' UTR of TRIM56 (siT56-UTR). Note that (1) in -tet cells only endogenous, untagged TRIM56 was detected, while in +tet cells both forms of TRIM56 were visible. Densitometry analysis of the immunoblotting data show that the total TRIM56/actin arbitrary units for +tet cells were 12-fold of that for -tet cells; (2) the siT56-UTR merely silenced the expression of the endogenous TRIM56 transcript but not that of the exogenously introduced (tet-inducible) TRIM56 which expressed only the coding region of the gene. (D) HEK293-FIT-T56 WT/Mut cells with (+tet) or without (-tet) tet were co-transfected siT56-UTR or the negative control siRNA (Ctrl), as well as a TLR3-encoding vector, $p R L-T K$ and IFN- $\beta$-luc, followed by mock- and poly-I:Ctreatment (for $8 \mathrm{~h}$ ) and dual luc reporter assay. Statistical analysis was performed between WT TRIM56 and vector or mutant TRIM56 after poly-I:C treatment in (A), between poly-I:C-treated -tet and +tet cells in (B) or (D), or between ctrl and siT56-UTR groups in (C) or (D). Single, double and triple asterisks denote that statistical differences exist with a $P$ value of $<0.05,<0.01$ and $<0.001$, respectively. N.S., not statistically significant. 


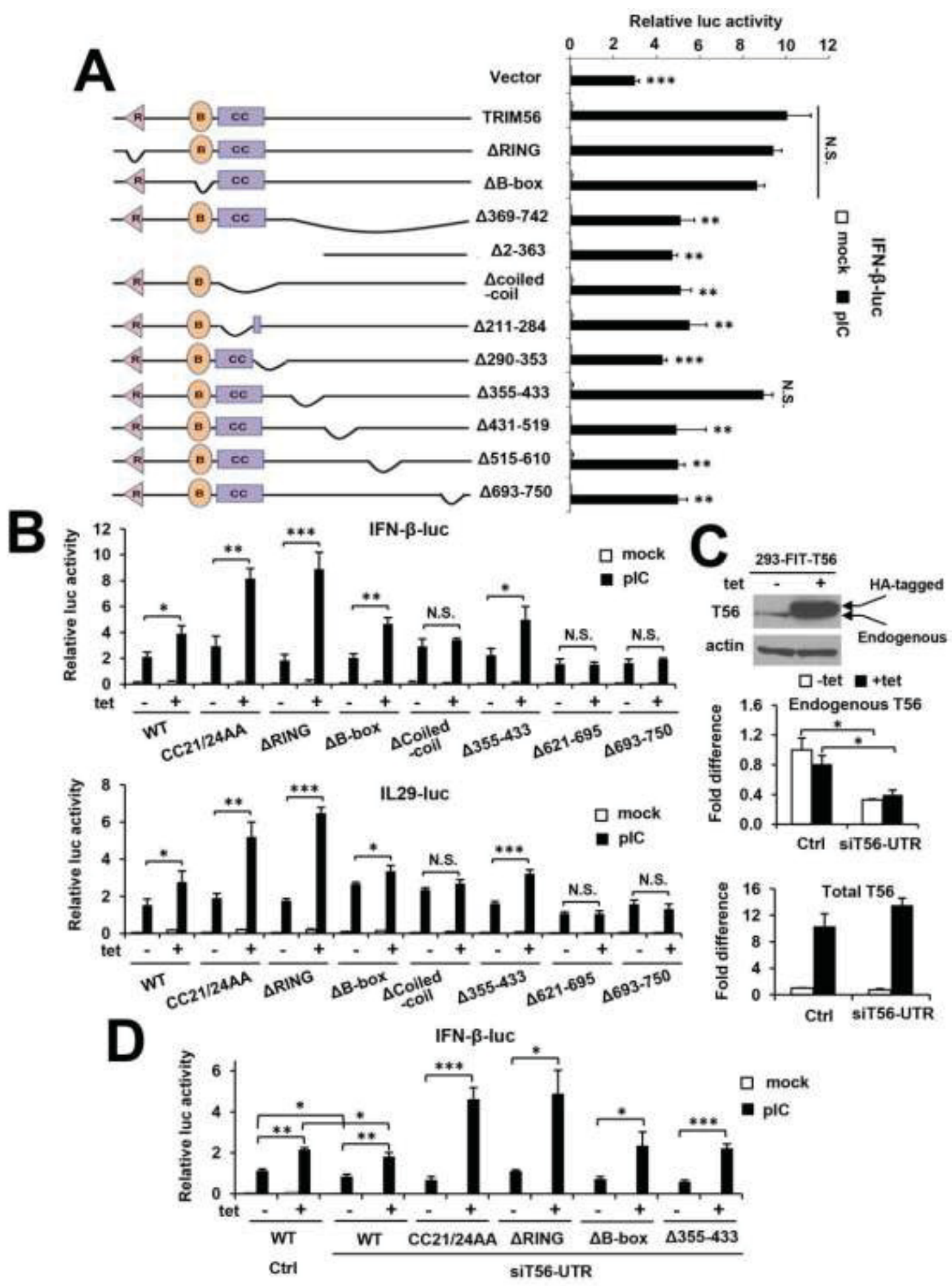


exogenously introduced HA-tagged TRIM56 in cells cultured in the presence of tet (+tet) is $~ 10-15$-fold higher than the endogenous TRIM56 transcript level (in cells cultured in the absence of tet (-tet)). The protein expression data are in line with the mRNA data, as shown in Figure 5-2C (top). When I efficiently silenced the expression of endogenous WT TRIM56 by transfecting cells with an siRNA (siT56-UTR) specifically targeting the 3' UTR of the endogenous TRIM56 transcript (Figure 5-2C), expression of the $\Delta$ B-box or $\Delta 355-433$ mutant was still able to augment poly-I:C-induced IFN-beta promoter activity (Figure 5-2D). Similar observation was also made when performing this assay with IL-29-luc (data not shown). These data confirm that the B-box and aa 355-433 are dispensable for the function of TRIM56 in promoting TLR3-mediated, IRF3-dependent signaling. I found a similar TRIM56 domain requirement profile for modulation of TLR3-mediated NF- $\kappa$ B activation (Figure 5-3), which is in agreement with our earlier finding that TRIM56 acts on the TLR3 pathway prior to the bifurcation of NF- $\kappa \mathrm{B}$ and IRF3.

\section{Putative Phosphorylation Sites S471, S475 and S710 Are Required for TRIM56- mediated Positive Regulation of TLR3 Signaling}

The C-terminal tail of TRIM56 has been shown to be indispensable for modulation of TLR3 signaling. Recently I have found there are four conserved serines in the C-terminal portions of human and murine TRIM56 (Figure 5-4A), which have been identified as phosphorylation sites in previous global proteomic studies (78-83). Specifically, the cluster of three putative phosphorylation sites (S469, S471 and S475) and S710 fall into aa 431-519 and aa 693-750, respectively, regions found to be essential for TRIM56's ability to augment TLR3 signaling (Figures 5-2A and 5-3; 39).

To determine if residue S710 plays a role in modulation of TLR3 signaling, vectors encoding TRIM56-V5 bearing S710A and S710D substitutions, respectively, were constructed in pCDNA3.1 backbone. These S710 mutants were expressed at comparable levels to that of WT TRIM56 or $\Delta 693-750$ in transfected cells as determined by immunoblotting (Figure 5-4B). I found that S710A mutation significantly $(P<0.01)$ reduced TRIM56-mediated up-regulation of poly-I:C-stimulated IFN- $\beta$ promoter activity in HEK293-TLR3 cells to an extent similar to the $\Delta 693-750$ deletion, suggesting TRIM56-S710 is important for positive regulation of TLR3 signaling (Figure 5-4C, left). Similarly, an alanine mutation (S689A) on mTRIM56-S689, the counterpart of hTRIM56 S710, also significantly impaired mTRIM56's ability to heighten TLR3 signaling (Figure 5-4C, right). To further investigate the role of putative phosphorylation on TRIM56-S710 in regulating TLR3 signaling, I expressed WT TRIM56 and the S710 mutants in HeLa-shT56 093 (clone \#9) cells stably transduced with an shRNA specifically targeting the 3'UTR of TRIM56 transcripts, which were not present in WT and mutant TRIM56 expression constructs. As shown in Figure 5-4D (left), I confirmed the knockdown efficiency of endogenous TRIM56 in HeLa-shT56 093 (clone \#9) cells (compare lane 9 with lane 1 or 2). Consistent with this, as shown in Figure 5-4D (right), poly-I:C-stimulated IFN- $\beta$ promoter activity significantly diminished in HeLa-shT56 093 cells 


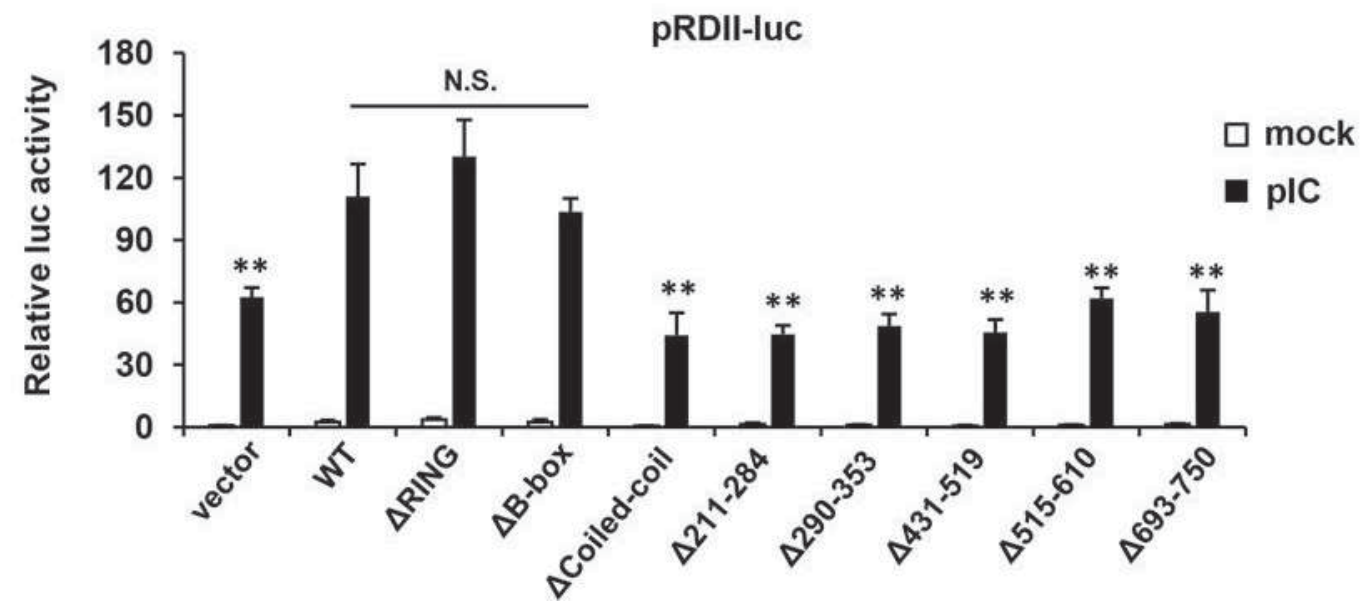

Figure 5-3. TRIM56's coiled-coil domain and residues 431-610, but not the B-box or residues 355-433, are critical for its potentiation of TLR3-mediated NF- $\mathrm{KB}$ dependent signaling pathway.

HEK293-TLR3 cells were co-transfected with an empty vector or vectors encoding WT and various mutant TRIM56-V5, as well as internal control plasmid pRL-TK and NF- $\mathrm{B}$ responsive reporter plasmid PRDII-luc, followed by mock- or poly-I:C-treatment (for 8 h) and dual luc reporter assay. Statistical analysis was performed between WT TRIM56 and vector or mutant TRIM56 after poly-I:C treatment. Double asterisks denote that statistical differences exist with a $P$ value of $<0.01$. N.S., not statistically significant. 
Figure 5-4. Putative phosphorylation on TRIM56's C-terminal serines S471, S475 and $\mathrm{S} 710$ is essential for its positive regulation of TLR3-mediated signaling pathway.

(A) Schematic representation of four conserved putative phosphorylation sites in human TRIM56 (S469, S471, S475 and S710) and mouse TRIM56. (B) Immunoblot analysis of the expression of vectors encoding WT TRIM56-V5 and its S710A, S710D and $\Delta 693$ 750 mutants (using mouse anti-V5 mAb) in HEK293 cells. Actin serves as a loading control. (C) HEK293-TLR3 cells were co-transfected with an empty vector or plasmids encoding WT or mutant hT56 (left part) or mT56 (upper right part), as well as internal control plasmid pRL-TK and reporter plasmid IFN- $\beta$-luc, followed by mock treatment or treatment with poly-I:C for $8 \mathrm{~h}$. Dual luc reporter assay was then performed to measure the promoter activation. (Lower right) Immunoblot analysis of expression of the vectors encoding WT mT56-V5 and its mutant S689A. (D) (Left) Immunoblot analysis of expression level of endogenous TRIM56 (using rabbit anti-TRIM56 pAb) in HeLa cells or those stably transduced with a non-targeting, scrambled control shRNA (Ctrl), or HeLa-shT56 cell clones stably transduced with a TRIM56 shRNA (\#093) which specifically targets the 3'UTR region of human TRIM56. Asterisk denotes a nonspecific band. (Right) HeLa-shT56 cells (clone \#9) and its knockdown control cells (Ctrl) were co-transfected with an empty vector or vectors encoding WT or mutant TRIM56 as well as internal control plasmid pRL-TK and reporter plasmid IFN- $\beta$-luc, followed by mock treatment or treatment with poly-I:C for $8 \mathrm{~h}$. Dual luc reporter assay was then performed to measure the promoter activation. (E) (upper part) Human hepatoma Huh7.5-TLR3 cells ectopically expressing TLR3 were co-transfected with an empty vector or vectors encoding WT or mutant TRIM56 as well as internal control plasmid pRL-TK and reporter plasmid IFN- $\beta$-luc, followed by mock treatment or treatment with poly-I:C (for 8 h) and dual luc reporter assay. (Lower part) Immunoblot analysis of the expression of the vectors encoding WT TRIM56-V5 and its S469A, S471A or S475A single mutant and $\mathrm{S} 469 \mathrm{~A}+\mathrm{S} 471 \mathrm{~A}+\mathrm{S} 475 \mathrm{~A}$ triple mutant (using mouse anti-V5 mAb) in HEK293 cells. Statistical analysis was performed between WT TRIM56 and vector or mutant TRIM56 after poly-I:C treatment. Single, double and triple asterisks denote that statistical differences exist with a $P$ value of $<0.05,<0.01$ and $<0.001$, respectively. N.S., not statistically significant. 

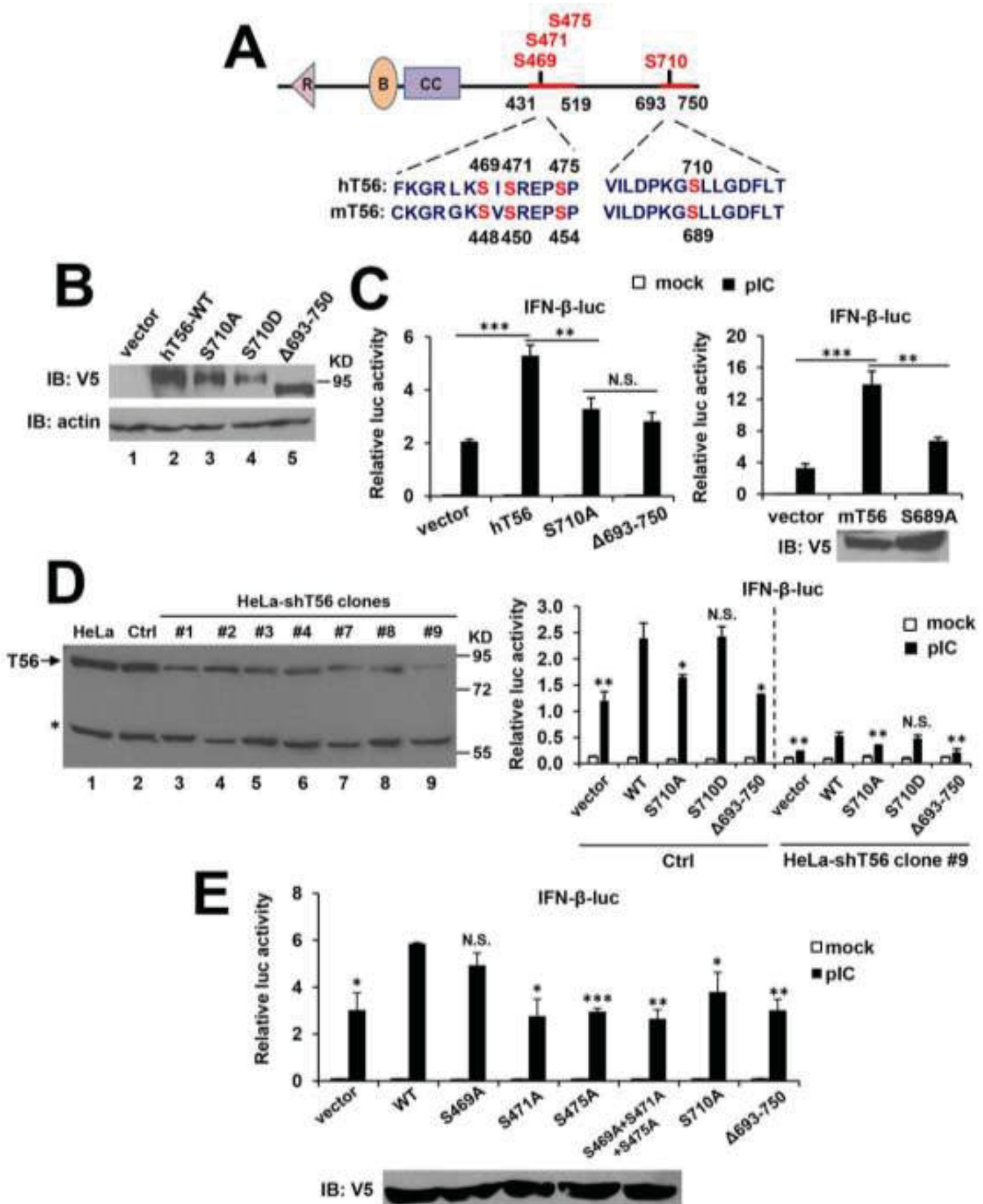
(clone \#9) compared with that in the knockdown control (Ctrl) cells. In HeLa-shT56 093 (clone \#9) cells, while the phospho-mimetic TRIM56-S710D mutant exhibited comparable activity to WT TRIM56 in promoting TLR3 signaling, the TRIM56-S710A mutant $(P<0.05)$ had a significantly impaired activity. Similar results were obtained in control HeLa cells.

Further, alanine screening mutagenesis (Figure 5-4E) suggested the S471A or S475A single mutant or $\mathrm{S} 469 \mathrm{~A}+\mathrm{S} 471 \mathrm{~A}+\mathrm{S} 475 \mathrm{~A}$ triple mutant failed to enhance TLR3 signaling $(P<0.05,<0.001$, and 0.01 , respectively), while the S469A mutant was only slightly impaired for such ability (statistically insignificant when compared with WT TRIM56), supporting a role for putative phosphorylation of TRIM56 at S471 and S475 in promoting TLR3 signaling. These findings show the importance of putative phosphorylation sites S710, S471 and S475 in TRIM56-mediated up-regulation of TLR3 signaling.

\section{Alanine Substitutions on the Important TRIM56's Putative Phosphorylation Sites and Deletion of the Coiled-coil Domain Undermine the TRIM56-mediated Enhancement of TLR3-dependent Antiviral Response}

I have shown that TRIM56 mutants on the putative phosphorylation sites and the coiled-coil domain impair TLR3-mediated IFN- $\beta$ promoter activation, which is critical for establishment of an antiviral state (39). Next I directly determined the impact of these mutations on TLR3-mediated establishment of an antiviral state. To this end, I established HEK293-FIT-derived cell lines with tet-inducible expression of HA-TRIM56 with alanine mutations on TRIM56's putative phosphorylation sites using the strategy we chose to create the 293-FIT-T56 cell line. These include cell lines with tet-inducible expression of S469A, S471A, S475A or S710A single mutant, and $\mathrm{S} 469 \mathrm{~A}+\mathrm{S} 471 \mathrm{~A}+\mathrm{S} 475 \mathrm{~A}$ triple mutant, respectively. As shown in Figure 5-5A, there was negligible expression of mutant HA-TRIM56 when these cell lines were cultured in the absence of tet (Figure 5-5A, odd-numbered lanes, data for S710A (-tet) not shown), while robust expression of mutant HA-TRIM56 of comparable levels to WT TRIM56 in HEK293-FIT-T56 cells, was detected in tet-containing medium (Figure 5-5A and lower panel of Figure 5-5B (including data for S710A + tet cells)). Next, HEK293-FIT-derived cells conditionally expressing WT and mutant HA-TRIM56 with (+tet) or without (-tet) tet were reconstituted with Flag-TLR3 by transient transfection of a TLR3-encoding plasmid (Figure 5-5B, lower), followed by poly-I:C stimulation to induce an antiviral state and then VSV-luc challenge. In mock-treated cells, regardless of cell type and tet addition status, VSV-luc replicated with similar efficiency as illustrated by the virusencoded luc activity (Figure 5-5B, upper), which is in line with our previous finding that TRIM56 itself had no influence on VSV replication (34). Poly-I:C stimulation induced an antiviral state in the cells without tet (-tet, i.e., no induction of WT or mutant TRIM56) to similar extent among WT and mutant TRIM56 cell lines, resulting in a $\sim 75 \%$ reduction in VSV-luc replication compared with unstimulated cells. By contrast, WT TRIM56 induction in 293-FIT-T56 (+tet) cells exhibited significantly $(P<0.05)$ more inhibition of VSV-luc replication (88\%), consistent with our earlier finding that TRIM56 potentiates the dsRNA-stimulated, TLR3-dependent antiviral response (39). This phenomenon was 
Figure 5-5. TRIM56's coiled-coil domain and C-terminal putative phosphorylation sites S710, S471 and S475 are important for the establishment of an antiviral state by TLR3 ligand.

(A) Generation of HEK293-FIT-derived cells that conditionally express individual TRIM56 mutants on the putative phopho-sites in a tet-inducible manner. Immunoblot analysis of expression of WT HA-TRIM56 and various mutant TRIM56 (using mouse anti-HA mAb) in 293-FIT-T56 WT/Mut cells with (+tet) or without (-tet) tet treatment for $48 \mathrm{~h}$. Actin serves as a loading control. (B) (Upper) HEK293-FIT-derived cells conditionally expressing WT HA-TRIM56 and its mutants at the indicated putative phospho-sites with (+tet) or without (-tet) tet, were reconstituted with TLR3 and mockstimulated or stimulated by poly-I:C, followed by infection with VSV-luc (MOI = 0.1). At $6 \mathrm{~h}$ postinfection, cells were lysed for firefly luciferase assay. (Lower) Immunoblot analysis of expression of the induced WT and mutant HA-TRIM56 (using mouse anti-HA $\mathrm{mAb}$ ) and reconstituted Flag-tagged TLR3 (using mouse anti-flag mAb) in the tested 293-FIT-T56 WT/Mut cells before VSV-luc infection. (C) HEK293-FIT-derived cells conditionally expressing WT HA-TRIM56 and its coiled-coil domain deletion mutant ( $\Delta$ coiled-coil) with (+tet) or without (-tet) tet, were reconstituted with TLR3 and mockstimulated or stimulated by poly-I:C, followed by infection with VSV-luc (MOI = 0.1). At $4 \mathrm{~h}$ postinfection, cells were lysed for firefly luciferase assay. (Lower) Immunoblot analysis of expression of the induced WT HA-TRIM56 and its $\Delta$ Coiled-coil mutant (using mouse anti-HA mAb) in the tested 293-FIT-T56 WT/ $\Delta$ Coiled-coil cells before VSV-luc infection. Statistical analysis was performed between poly-I:C-treated -tet and + tet cells. Single and double asterisks denote that statistical differences exist with a $P$ value of $<0.05$ and $<0.01$, respectively. N.S., not statistically significant. 


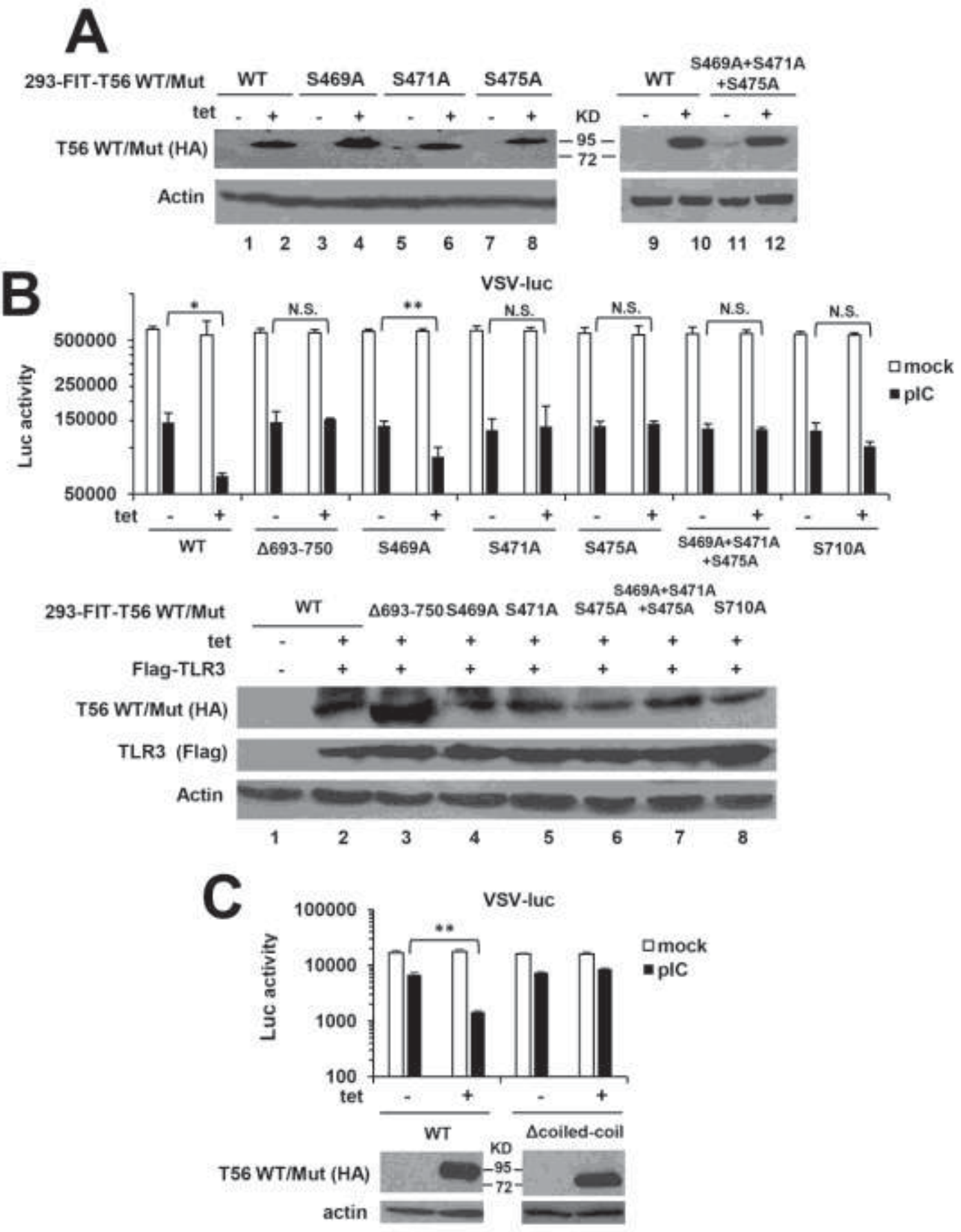


also found in TRIM56-S469A expressing cells (+tet) which had 85\% decrease in VSVluc replication, suggesting that TRIM56-S469A retains the ability to promote the TRIM56-TLR3-dependent antiviral response. On the contrary, expression of $\Delta 693-750$, S471A, S475A, S469A+S471A+S475A or S710A mutants failed to promote a poly-I:Cstimulated, TRIM56-TLR3-dependent antiviral response. There was no further statistically significant decrease in VSV-luc replication in poly-I:C-treated, +tet mutant cells as compared with poly-I:C-treated, -tet mutant cells (Figure 5-5B, upper). This could also be said for cells conditionally expressing the $\Delta$ coiled-coil mutant, since the 293-FIT- $\Delta$ coiled-coil cells cultured in the presence of tet (+tet) lost the ability to enhance poly-I:C-stimulated antiviral response against VSV-luc (Figure 5-5C). These observations show TRIM56's putative phosphorylation sites S471, S475 and S710 and its coiled-coil domain play critical roles in promoting TLR3-dependent antiviral responses.

\section{TRIM56's Putative Phosphorylation Site S710 on the C-terminus, but Not the Coiled-coil Domain, Is Critical for Its Association with TRIF}

I next sought to characterize the mechanism(s) by which TRIM56's putative phosphorylation site and coiled-coil domain help TRIM56 to promote TLR3 signaling. TRIM56 was reported to promote TLR3 signaling by forming a complex with TRIF (39). Given the fact that $\mathrm{S} 710$ falls into the region (aa 621-750) responsible for its association with TRIF, I asked if this putative phosphorylation site is important for TRIM56-TRIF interaction. Co-IP experiments showed that the S710A mutant abolished the TRIM56TRIF interaction similar to the $\Delta 693-750$ TRIM56 mutant (Figure 5-6A). Since the S710A mutant lost the ability to augment poly-I:C-induced IFN transcription (Figure 5-4) and host antiviral response (Figure 5-5B), I concluded that TRIM56's putative phosphorylation site S710 may affect the TRIM56 interaction with TRIF. Next I asked if the coiled-coil domain, which was found to be important for positive regulation of TLR3 signaling was also indispensable for TRIM56-TRIF association. As shown in the co-IP experiment with 293-FIT-T56 WT/Mut cells (Figure 5-6B), the $\Delta$ Coiled-coil mutant retained the ability to form a complex with TRIF, suggesting that the coiled-coil domain is not required for the TRIM56-TRIF association. Interestingly, this experiment also showed that aa 355-433 does not mediate the interaction with TRIF either, since the $\Delta 355-433$ mutant can still interact with TRIF.

\section{Discussion}

It has been shown that TRIM56 possesses the ability to promote TLR3-mediated IRF3-dependent antiviral signaling and IFN induction. Interestingly, TRIM56 knockdown suppressed TLR3-mediated induction of RANTES and IP-10 in hepatitis C virus (HCV) infected hepatoma cells (39), chemokines that are under coordinate control of NF- $\kappa$ B and IRF3 (84). The present study directly demonstrates that TRIM56 positively regulates TLR3-mediated NF- $\mathrm{KB}$ activation (Figure 5-1B), in addition to its effect on IRF3 activation (39). These data suggest that TRIM56 acts at a level prior to the bifurcation of IRF3 and NF- $\kappa \mathrm{B}$, and are consistent with our previous data showing that 


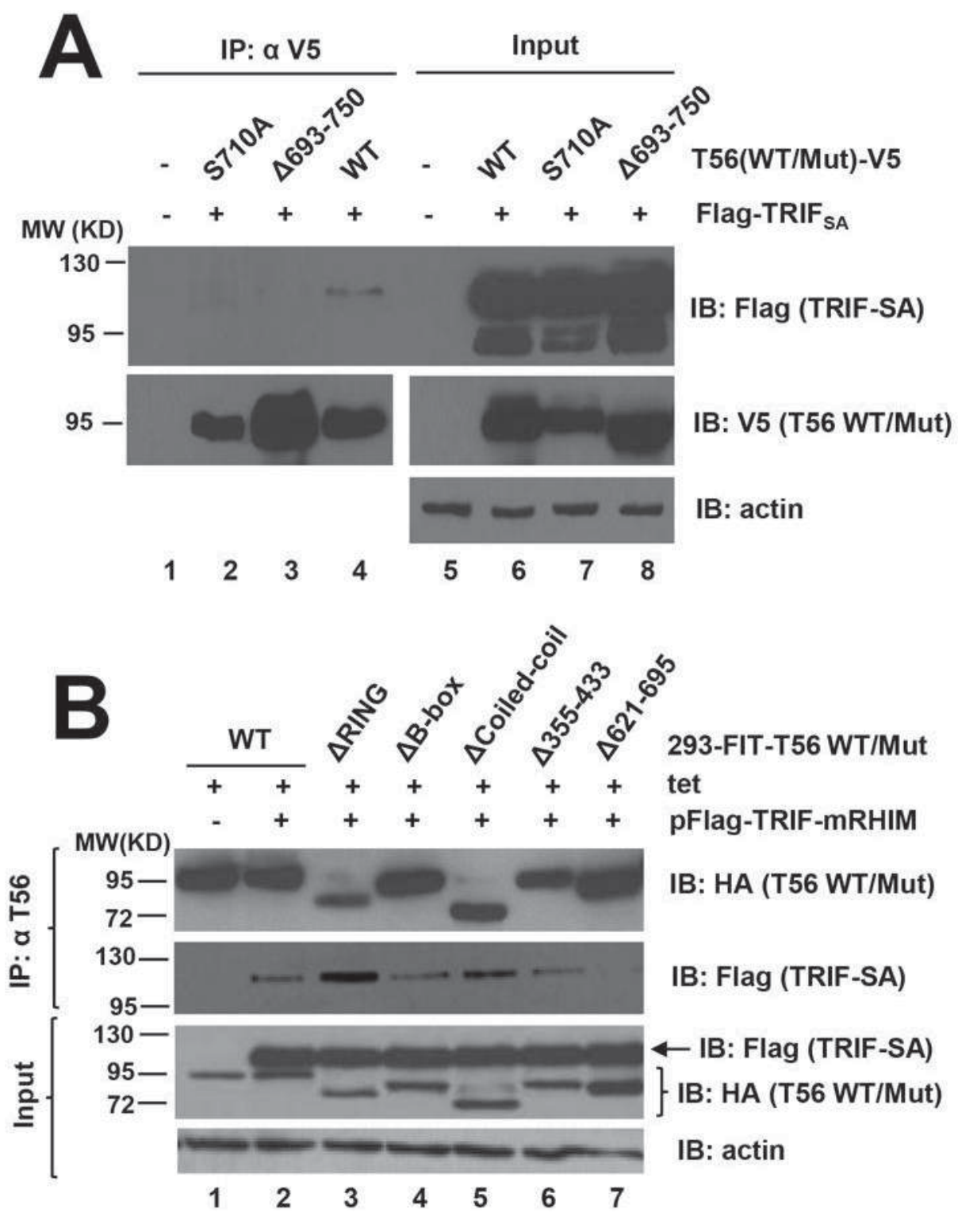

Figure 5-6. TRIM56's putative phospho-site S710, but not its coiled-coil domain, is important for its interaction with TRIF.

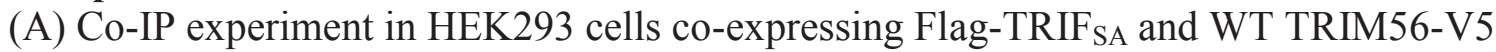
or various mutant TRIM56-V5. Cell lysates were immunoprecipitated (IP) with anti-V5, followed by immunoblotting (IB) with anti-Flag or anti-V5. The right blot showed

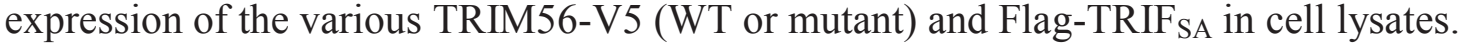

(B) Co-IP experiment in HEK293-FIT-T56 WT/mut cells co-expressing Flag-TRIF SA $_{\text {and }}$ WT or various mutant HA-TRIM56 (by addition of tet (+tet)). Cell lysates were immunoprecipitated (IP) with anti-TRIM56, followed by immunoblotting (IB) with antiFlag or anti-HA. The bottom blot showed expression of the various HA-TRIM56 (WT or

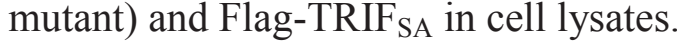


TRIM56 likely promotes TLR3 signaling by associating with TRIF. As additional evidence supporting this notion, we show here that endogenous TRIM56 and TRIF proteins formed a complex at an early stage after TLR 3 engagement, prior to induction of ISG56, an IRF3-target gene (Figure 5-1). The observation that TRIM56 promotes both IRF3 and NF- $\mathrm{KB}$ arms of TLR3 signaling suggests that it may regulate IFN antiviral responses as well as inflammatory chemokine/cytokine responses activated through this innate immune pathway.

In this study, I have comprehensively dissected the molecular determinants governing the ability of TRIM56 to positively regulate the TLR3 signaling pathway. I found that TRIM56's coiled-coil domain and an extended C-terminal portion (aa 431-610) are critical for promoting TLR3 signaling (Figures 5-2 and 5-3), while the B-box and a central region downstream of the Coiled-coil domain (aa 355-433) are not. Within the important C-terminal region, I identified three conserved, putative phosphorylation sites, i.e., S471, S475 and S710, as indispensable for TRIM56-mediated up-regulation of TLR3 signaling (Figure 5-4). Importantly, these findings were observed in different cell types and expression/silencing systems. Moreover, the effects on IFN promoter activation were recapitulated by VSV-Luc-based assay directly gauging TLR3-dependent establishment of an antiviral state. (Figure 5-5). Together, our data reveal novel insights into the mechanism of TRIM56 augmentation of the TLR3-dependent antiviral response and highlights a role for TRIM56 scaffolding and phosphorylation in positive regulation of TLR3 signaling.

In the extended C-terminal region now identified to be required for up-regulation of TLR3 signaling, aa 515-610 account for half of the NHL-like repeats of TRIM56 (aa 497-754, Figure 4-9), a barrel-like $\beta$-propeller structure which may be important for protein-RNA or protein-protein interaction (36). On the other hand, I found that putative phosphorylation sites S471 and S475 within aa 431-519 and S710 on the C-terminal end are important for TRIM56 to potentiate TLR3-dependent IFN- $\beta$ promoter activation and establishment of an antiviral state (Figures 5-4 and 5-5). Furthermore, S710A completely abolished TRIM56-TRIF interaction (Figure 5-6). Others studies of post-translational modification of TRIMs were focused on ubiquitination due to the fact that TRIMs bear E3 ligase activity and they tend to catalyze the self-reactions that are believed to be important for their functions $(29,34,37)$. Recently tyrosine phosphorylation of the E3 ubiquitin ligase TRIM21 has been reported to be critical for TLR3/4-mediated positive regulation of IRF3 activation and IFN induction (85). Interestingly, ProtScale analysis indicates that aa 425-475 of TRIM56 is a highly hydrophilic region for TRIM56, which is in agreement with our prediction that phosphorylation occurs on S471 or/and S475.

This study also demonstrates that the coiled-coil domain is indispensable for TRIM56 promotion of TLR3 signaling (Figures 5-2, 5-3 and 5-5). The coiled-coil domains of TRIMs have been reported to mediate dimerization of TRIM molecules (66). Interestingly, I observed that a coiled-coil domain deletion mutant ( $\Delta$ Coiled-coil) was still able to associate with TRIF (Figure 5-6B). This suggests that the TRIM56-TRIF interaction does not require TRIM56 dimerization. Rather, I propose that the TRIM56 coiled-coil domain may serve as a scaffold that recruits and interacts with other important 
signaling components, thereby accelerating signal transduction. However, further experiments are needed to confirm that the coiled-coil domain is indispensable for TRIM56 oligomerization. Also, further investigation is still needed to clarify the previse role of the TRIM56 coiled-coil domain and its possible scaffolding function in positive regulation of TLR3 signaling. 


\section{CHAPTER 6. DISCUSSION}

\section{The Distinct Domain Requirements for TRIM56's Anti-flaviviruses and Anti- HCoV-OC43 Actions May Reflect Different Molecular Mechanisms That TRIM56 Adopts to Restrict Different Positive-strand RNA Viruses}

In this body of work, I have demonstrated that human TRIM56 is an antiviral host factor against diverse positive-strand RNA viruses. Specifically, TRIM56 exhibits antiviral activities against two medically important flaviviruses (YFV and DENV2) and a human coronavirus (HCoV-OC43) (Figures 3-1 and 3-2). Further, I have shown that the E3 ligase activity is a shared requirement for the antiviral actions of TRIM56 against the two flaviviruses and $\mathrm{HCoV}-\mathrm{OC} 43$, and that while dispensable for restricting $\mathrm{HCoV}$ OC43, the integrity of the C-terminal portion of TRIM56 is additionally required for the anti-flavivirus functions (Figures 3-6, 3-7 and 3-9). I also found that TRIM56 suppresses intracellular viral RNA replication of yellow fever and dengue viruses but may target the packaging or release steps in $\mathrm{HCoV}-\mathrm{OC} 43$ life cycle (Figure 3-10).

The findings concerning the differing domain requirements for the novel antiviral actions of TRIM56 suggest differences in the antiviral mechanisms against different viruses. By virtue of the E3 ligase activity, TRIM56 might modulate posttranslational modification(s) of one or more viral proteins and host factors to suppress positive-strand RNA virus replication (34). Given its broad antiviral activities against positive-strand RNA viruses, I favor the hypothesis that host factors are involved. The target proteins for TRIM56 E3 ligase during infections with flaviviruses (YFV and DENV) and HCoVOC43 will need further studies. TRIM56 promotes ubiquitination (34), the best-known enzymatic activity for most TRIMs $(29,31,77,86)$. It is not known, however, how this modification of the target proteins may be responsible for the antiviral effects, including, but not limited to, promoting their degradation or altering their intracellular trafficking. It is also possible that TRIM56 antiviral function works by promoting Ub-like modifications such as sumoylation and ISGylation, as demonstrated with TRIM28 and TRIM25 $(87,88)$.

Moreover, the dichotomy in the molecular determinants governing TRIM56's antiflaviviruses and anti-HCoV-OC43 functions is that the former is additionally dependent upon TRIM56's C-terminal portion. I speculate that this might accommodate the different replication strategies between flaviviruses and coronaviruses. Given that the C-terminal region mediates the interactions of TRIM56 with STING, N-terminal protease of BVDV, and TRIF $(34,39,58)$, protein-protein interactions via the $\mathrm{C}$-terminal portion may participate in TRIM56's antiviral actions against YFV and DENV2. Besides facilitating presentation of specific substrate to E2 conjugating enzyme, TRIM56's C-terminal part may form a complex with flaviviral protein(s) and/or perhaps flavivirus-specific host factors in the cytoplasm, in ways that hinder viral RNA replication. I favor the notion that host factors are likely targeted by TRIM56 given that we did not find any BVDV replicase proteins to interact with TRIM56 in a previous study (34). In contrast to the proposed canonical TRIM E3 ligase model in which the RING domain and C-terminal 
portion work in concert $(29,65)$, the E3 ligase activity as the sole prerequisite for TRIM56's anti-HCoV-OC43 effect might suggest a reliance on some other co-factors to accomplish substrate presentation in lieu of TRIM56's C-terminal portion, which will require further investigation. Obviously, identifying the interaction partners of TRIM56 holds the key to understanding the exact antiviral mechanisms. Studies to this end are underway in our laboratory.

\section{Possible Molecular Mechanisms Underlying Which C-terminal End of TRIM56 Undermines Influenza Virus RNA Replication}

In this body of work, I have demonstrated that TRIM56 compromised propagation of both influenza A and B viruses by suppressing viral RNA replication (Figures 4-1, 4-2, 4-6 and 4-7), which only requires TRIM56's C-terminal 63 residues (Figures 4-3 and 44). Moreover, expression of this short C-terminal segment (CTT135 or CTT63) restricts influenza viruses as effectively as WT TRIM56 (Figure 4-5). In line with its function in curbing influenza virus RNA replication, which takes place in the nucleus, I found that a fraction of TRIM56 moved into the nucleus after influenza virus infection (Figure 4-8). This is also true for CTT63. These findings provide insights into the mechanisms by which TRIM56 curbs these medically-important negative-strand RNA viruses.

Precisely how the C-terminal tail portion of TRIM56 impedes influenza viral RNA synthesis is unclear. The C-terminal region of TRIM56 has been shown to interact with viral and cellular proteins imparting various biological functions, as exemplified by the TRIM56 association with the N-terminal protease of BVDV (34), TRIF (39), and STING (58). In light of this, TRIM56 may associate with a component(s) of viral RNP or a proviral host factor(s) recruited to the RNP complex, interfering with the partner's role in viral RNA synthesis. This notion, to some extent, is supported by the confocal data (Figure 4-8), which suggest co-localization of TRIM56 with NP or M proteins after IAV infection. In addition, in co-IP assays performed in HEK293-TLR3-YFP cells overexpressing Flag-HA-tagged TRIM56 (293-T3Y-FH-TRIM56), and I observed that TRIM56 formed a complex with NP during IAV infection (data not shown). More experiments are still required to confirm the TRIM56-NP interaction and determine its biological consequences.

However, another possibility is that TRIM56 interacts with influenza viral RNAs, altering their incorporation into the viral transcription/replication complex and/or their stability. Though TRIM56 was not previously identified in influenza virus particles (89), the possibility of TRIM56 associating with mRNAs of influenza virus or proviral cellular factors and regulating their translation also cannot be ruled out. Intriguingly, the Cterminal region of TRIM56 (residues 521-748, see Figures 4-5A and 4-9) exhibits sequence homology with NHL repeats based on a search of the NCBI conserved domain database. Sequence alignment of TRIM56 with several well-characterized TRIM-NHL proteins, TRIM2, TRIM3, TRIM32 and TRIM71, also showed conservation on numerous consensus residues shaping the NHL repeats (Figure 4-9) $(75,76)$. The NHL repeats fold into a barrel-like $\beta$-propeller structure, whose partial surface is positively charged and 
could directly interact with the phosphate backbone of RNA, which is negatively charged. As an example, TRIM71 can associate with miRNAs and mRNAs and regulate translation and mRNA metabolism (90). Obviously, further investigation is required to elucidate how TRIM56 hinders influenza virus RNA synthesis, knowledge of which will provide new insights into factors and molecular interactions controlling this vital step in influenza virus life cycle.

\section{Dissect the Molecular Mechanisms Dictating TRIM56-mediated Positive Regulation of the TLR3 Signaling Pathway}

In this body of work, I studied the TRIM56 molecular determinants that govern the positive regulation of TLR3-mediated IRF3 and NF- $\kappa$ B activation. Confirming our previous finding that TRIM56 formed a complex with TRIF in an overexpression setting (39), I found that an association of endogenous TRIM56 and TRIF proteins occurs at an early stage after TLR3 engagement (Figures 5-1), prior to dsRNA-induced ISG56 expression through this pathway. Detailed domain mapping suggests that TRIM56's coiled-coil domain and aa 431-610 were required for augumentation of TLR3 signaling (Figures 5-2 and 5-3). Further, three conserved, putative phosphorylation sites S471, S475 and S710 were found to be important for up-regulation of TLR3-mediated signaling (Figure 5-4). Concordant with this, I demonstrated that the putative phosphorylation sites S471, S475 and S710 as well as the coiled-coil domain are essential for TLR3-mediated establishment of an antiviral state (Figure 5-5). Given that the coiled-coil domain confers TRIMs with scaffolding function and that post-translational modification, such as phosphorylation, play an important role in regulating protein function $(34,66,85)$, our data suggest that further studies of TRIM56's scaffolding function and phosphorylation status at S471, S475 and S710 after TLR3 engagement are important to better understand the precise molecular mechanisms by which TRIM56 positively regulateTLR3 signaling.

Although TLR3 contributes to sensing viral invasion and facilitating the establishment of an antiviral state during certain viral infections, excessive and unregulated immune signaling through this PRR may lead to overwhelmingly harmful inflammatory responses detrimental to the host $(4,84)$. I have demonstrated that TRIM56

promotes TLR3-mediated NF- $\kappa$ B activation, supporting that TRIM56 might play a role in inflammatory cytokine/chemokine responses. It would be interesting to examine whether TRIM56-regulated TLR3 signaling leads to heightened up-regulation of proinflammatory cytokines/chemokines, which are NF- $\mathrm{BB}$-dependent. Another intriguing question is how host cells tune down TRIM56-promoted TLR3 signaling after PAMPs have been sensed and this pathway has been activation. Indeed, the dynamic interaction between endogenous TRIM56 and TRIF protein (Figure 5-1C) suggests the association between TRIM56 and the TLR3 adaptor protein may be tightly regulated.

To further investigate exactly how the scaffolding function conferred on TRIM56 by its coiled-coil domain contributes to positive regulation of TLR3 signaling, it would be worthwhile to correlate TRIM56's scaffolding function with its ability to promote the TLR3 signaling. This aspect could be examined by determining whether the $\Delta$ Coiled-coil 
mutant that fails to promote TLR3 signaling loses the scaffolding function. To this end, I have performed native PAGE and subsequent immunoblot analysis demonstrating that WT TRIM56 molecules self-associate to form homodimers but the $\Delta$ Coiled-coil mutant failed to do so (data not shown). Moreover, to directly demonstrate that the $\Delta$ Coiled-coil mutant loses the ability to associate with each other, V5-tagged, WT TRIM56 or $\Delta$ Coiled-coil mutant will be co-transfected into HEK293 cells with GFP-tagged WT TRIM56, followed by IP using anti-V5 and immunoblot analysis for GFP-tagged WT TRIM56. Further, key residues in TRIM56's coiled-coil domain, which are critical for self-association or oligomerization, should be identified and tested for their necessity for TRIM56-mediated augmentation of TLR3 signaling. Recent crystallographic and biochemical analyses have demonstrated that L252 and E256 in the TRIM25 coiled-coil domain are two important residues stabilizing the elongated antiparallel hairpin dimer formed by the coiled-coil domains (66). Interestingly, that leucine residue is well conserved among different TRIMs including TRIM56, in which it is at 266 position (L266). In the future, it will be interesting to determine the effect of L266 mutation on TRIM56 dimerization and TRIM56-mediated enhancement of TLR3 signaling..

My data suggest that putative phosphorylations on S471, S475 and S710 may be important for the ability of TRIM56 to promote TLR3 signaling. Obviously, directly examining this post-translational modification at these residues is warranted. Along this line, our attempts to detect phosphorylation of TRIM56 at S471, S475 and S710 following TLR3 engagement by performing IP using anti-TRIM56 antibody followed by immunoblot analysis using anti-phosphoserine antibody have thus far failed, due to the poor specificity and sensitivity of the available anti-phosphoserine antibodies. To solve this problem, we have custom-made phospho-specific antibody recognizing p-S471 or pS475 and experiments with these antibodies are under way.

\section{Conclusion}

I unveiled the spectrum and specificity of the antiviral activities of TRIM56 against distinct positive-strand RNA viruses such as yellow fever and dengue viruses as well as human coronavirus-OC43, broadening the scope of the roles this TRIM plays in antiviral immunity (Figures 3-1 and 3-2). Equally important, I showed that TRIM56 accommodates these previously unrecognized antiviral functions via overlapping and distinct molecular determinants, which dictate shared and disparate antiviral actions (Figures 3-6, 3-7 and 3-9). These new revelations provide novel insights into the detailed antiviral mechanisms of TRIM56 (Figure 3-10) and raise the possibility of targeting this TRIM for development of broad antivirals.

Furthermore, I identified TRIM56 as an intrinsic cellular restriction factor of influenza $\mathrm{A}$ and $\mathrm{B}$ viruses (Figures 4-1 and 4-2) and described a novel, E3 ligase-independent antiviral mechanism that targets influenza viral RNA synthesis (Figures 4-3 through 4-7). Our data delineate novel antiviral properties of TRIM56 against orthomyxoviruses and may open new avenues to developing influenza antivirals. 
Last but not least, I confirmed that TRIM56 is a positive regulator of TLR3-mediated NF- $\kappa \mathrm{B}$ signaling (Figure 5-1) and demonstrated that a large C-terminal portion and the coiled-coil domain of TRIM56 are both essential for promoting TLR3 signaling

(Figures 5-2 and 5-3). Further, I demonstrated a requirement for 3 putative phosphorylation sites, i.e., S471, S475 and S710 for TRIM56-mediated augmentation of TLR3 signaling. S710 may be an important binding site for TRIM56-TRIF interaction (Figures 5-4 through 5-6), or phosphorylation at this residue is critical for maintaining the proper conformation of TRIM56 C-terminus. Furthermore, I demonstrated that TRIM56's coiled-coil domain is indispensable for TLR3-mediated establishment of an antiviral state (Figure 5-5), confirming the signaling data. Together, my data revealed novel insights into the mechanism dictating TRIM56's augmentation of TLR3-dependent antiviral response and highlighted role for TRIM56 scaffolding and phosphorylation in positive-regulation of TLR3 signaling. 


\section{LIST OF REFERENCES}

1. Kawai T, Akira S. 2006. Innate immune recognition of viral infection. Nat Immunol 7:131-137.

2. Kawai T, Akira S. 2010. The role of pattern-recognition receptors in innate immunity:update on Toll-like receptors. Nat Immunol 11:373-384.

3. Yoneyama M, Fujita T. 2010. Recognition of viral nucleic acids in innate immunity. Rev Med Virol 20:4-22.

4. Lester SN, Li K. 2014. Toll-Like receptors in antiviral innate immunity. J Mol Biol 426:1246-1264.

5. Baumann JG. 2006. Intracellular restriction factors in mammalian cells-an ancient defense system finds a modern foe. Curr HIV Res 4:141-168.

6. Tsai YT, Chang SY, Lee CN, Kao CL. 2009. Human TLR3 recognizes dengue virus and modulates viral replication in vitro. Cell Microbiol 11:604-615.

7. Daffis S, Samuel MA, Suthar MS, Gale M Jr, Diamond MS. 2008. Toll-like receptor 3 has a protective role against West Nile virus infection. J Virol 82:10349-10358.

8. Hardarson HS, Baker JS, Yang Z, Purevjav E, Huang CH, Alexopoulou L, Li N, Flavell RA, Bowles NE, Vallejo JG. 2007. Toll-like receptor 3 is an essential component of the innate stress response in virus-induced cardiacinjury. Am J Physiol Heart Circ Physiol 292:H251-H258.

9. Stack J, Haga IR, Schröder M, Bartlett NW, Maloney G, Reading PC, Fitzgerald KA, Smith GL, Bowie AG. 2005. Vaccinia virus protein A46R targets multiple Toll-likeinterleukin-1 receptor adaptors and contributes to virulence. J Exp Med 201:10071018.

10. Harte MT, Haga IR, Maloney G, Gray P, Reading PC, Bartlett NW, Smith GL, Bowie A, O'Neill LA. 2003. The poxvirus protein A52R targets Toll-like receptor signaling complexes to suppress host defense. J Exp Med 197:343-351.

11. Li K, Foy E, Ferreon JC, Nakamura M, Ferreon AC, Ikeda M, Ray SC, Gale M Jr, Lemon SM. 2005. Immune evasion by hepatitis $C$ virus NS3/4A protease-mediated cleavage of the Toll-like receptor 3 adaptor protein TRIF. Proc Natl Acad Sci U S A102:2992-2997.

12. World Health Organization. 2014. Factsheet 211: influenza. World Health Organization, Geneva, Switzerland. 
13. Paddock CD, Liu L, Denison AM, Bartlett JH, Holman RC, Deleon-Carnes M, Emery SL, Drew CP, Shieh WJ, Uyeki TM, Zaki SR. 2012. Myocardial injury and bacterial pneumonia contribute to the pathogenesis of fatal influenza B virusinfection. J Infect Dis 205:895-905.

14. Nelson MI, Holmes EC. 2007. The evolution of epidemic influenza. Nat Rev Genet 8:196-205.

15. Palese P, Shaw ML. 2007. Orthomyxoviridae: the viruses and their replication, $p$ 1647-1689. In Knipe DM, Howley PM (ed), Fields virology. Lippincott Williams \& Wilkins, Philadelphia, PA.

16. Warren-Gash C, Smeeth L, Hayward AC. 2009. Influenza as a trigger for acute myocardial infarction or death from cardiovascular disease: a systematic review. Lancet Infect Dis 9:601-610.

17. Mamas MA, Fraser D, Neyses L. 2008. Cardiovascular manifestations associated with influenza virus infection. Int J Cardiol 130:304-309.

18. Davis MM, Taubert K, Benin AL, Brown DW, Mensah GA, Baddour LM, Dunbar S, Krumholz HM; American Heart Association; American College of Cardiology. 2006. Influenza vaccination as secondary prevention for cardiovascular disease: a science advisory from the American Heart Association/American College of Cardiology. Circulation 114:1549-1553.

19. Haq K, McElhaney JE. 2014. Immunosenescence: influenza vaccination and the elderly. Curr Opin Immunol 24:38-42.

20. Boulo S, Akarsu H, Ruigrok RW, Baudin F. 2007. Nuclear traffic of influenza virus proteins and ribonucleoprotein complexes. Virus Res 124:12-21.

21. Cros JF, García-Sastre A, Palese P. 2005. An unconventional NLS is critical for the nuclear import of the influenza A virus nucleoprotein and ribonucleoprotein. Traffic 6:205-213.

22. Le Goffic R, Pothlichet J, Vitour D, Fujita T, Meurs E, Chignard M, Si-Tahar M. 2007. Cutting Edge: Influenza A virus activates TLR3-dependent inflammatory and RIG-I-dependent antiviral responses in human lung epithelial cells. J Immunol 178:3368-3372.

23. Bernasconi D, Amici C, La Frazia S, Ianaro A, Santoro MG. 2005. The IkappaB kinase is a key factor in triggering influenza A virus-induced inflammatory cytokine production in airway epithelial cells. J Biol Chem 280:24127-24134. 
24. Pan HY, Yamada H, Chida J, Wang S, Yano M, Yao M, Zhu J, Kido H. 2011. Upregulation of ectopic trypsins in the myocardium by influenza A virus infection triggers acute myocarditis. Cardiovasc Res 89:595-603.

25. Talon J, Horvath CM, Polley R, Basler CF, Muster T, Palese P, García-Sastre A. 2000. Activation of interferon regulatory factor 3 is inhibited by the influenza A virus NS1 protein. J Virol 74:7989-7996.

26. Kumar N, Xin ZT, Liang Y, Ly H, Liang Y. 2008. NF-kappaB signaling differentially regulates influenza virus RNA synthesis. J Virol 82:9880-9889.

27. Flory E, Kunz M, Scheller C, Jassoy C, Stauber R, Rapp UR, Ludwig S. 2000. Influenza virus-induced NF-kappaB-dependent gene expression is mediated by overexpression of viral proteins and involves oxidative radicals and activation of IkappaB kinase. J Biol Chem 275:8307-8314.

28. Munir M. 2010. TRIM proteins: another class of viral victims. Sci Signal 3:jc2.

29. Rajsbaum R, García-Sastre A, Versteeg GA. 2014. TRIMmunity: The roles of the TRIM E3-ubiquitin ligase family in innate antiviral immunity. J Mol Biol 426:12651284.

30. Kawai T, Akira S. 2011. Regulation of innate immune signalling pathways by the tripartite motif (TRIM) family proteins. EMBO Mol Med 3:513-527.

31. Ozato K, Shin DM, Chang TH, Morse HC $3^{\text {rd }}$. 2008. TRIM family proteins and their emerging roles in innate immunity. Nat Rev Immunol 8:849-860.

32. Stremlau M, Owens CM, Perron MJ, Kiessling M, Autissier P, Sodroski J. 2004. The cytoplasmic body component TRIM5alpha restricts HIV-1 infection in Old World monkeys. Nature 427:848-853.

33. Di Pietro A, Kajaste-Rudnitski A, Oteiza A, Nicora L, Towers GJ, Mechti N, Vicenzi E. 2013. TRIM22 inhibits influenza A virus infection by targeting the viral nucleoprotein for degradation. J Virol 87:4523-4533.

34. Wang J, Liu B, Wang N, Lee YM, Liu C, Li K. 2011. TRIM56 is a virus- and interferon-inducible E3 ubiquitin ligase that restricts pestivirus infection. J Virol 85:3733-3745.

35. Liu B, Li NL, Wang J, Shi PY, Wang T, Miller MA, Li K. 2014. Overlapping and distinct molecular determinants dictating the antiviral activities of TRIM56 against flaviviruses and coronavirus. J Virol 88:13821-13835. 
36. Liu B, Li NL, Shen Y, Bao X, Fabrizio T, Elbahesh H, Webby RJ, Li K. 2016. The C-terminal tail of TRIM56 dictates antiviral restriction of influenza A and B viruses by impeding viral RNA synthesis. J Virol pii: JVI.03172-15.

37. Versteeg GA, Rajsbaum R, Sánchez-Aparicio MT, Maestre AM, Valdiviezo J, Shi M, Inn KS, Fernandez-Sesma A, Jung J, García-Sastre A. 2013. The E3-ligase TRIM family of proteins regulates signaling pathways triggered by innate immune patternrecognition receptors. Immunity 38:384-398.

38. Gack MU, Shin YC, Joo CH, Urano T, Liang C, Sun L, Takeuchi O, Akira S, Chen Z, Inoue S, Jung JU. 2007. TRIM25 RING-finger E3 ubiquitin ligase is essential for RIG-I-mediated antiviral activity. Nature 446:916-920.

39. Shen Y, Li NL, Wang J, Liu B, Lester S, Li K. 2012. TRIM56 is an essential component of the TLR3 antiviral signaling pathway. J Biol Chem 287:36404-36413.

40. Chen Z, Benureau Y, Rijnbrand R, Yi J, Wang T, Warter L, Lanford RE, Weinman SA, Lemon SM, Martin A, Li K. 2007. GB virus B disrupts RIG-I signaling by NS3/4A-mediated cleavage of the adaptor protein MAVS. J Virol 81:964-976.

41. Xie X, Wang QY, Xu HY, Qing M, Kramer L, Yuan Z, Shi PY. 2011. Inhibition of dengue virus by targeting viral NS4B protein. J Virol 85:11183-11195.

42. Wang P, Song W, Mok BW, Zhao P, Qin K, Lai A, Smith GJ, Zhang J, Lin T, Guan Y, Chen H. 2009. Nuclear factor 90 negatively regulates influenza virus replication by interacting with viral nucleoprotein. J Virol 83:7850-7861.

43. Wang N, Liang Y, Devaraj S, Wang J, Lemon SM, Li K. 2009. Toll-like receptor 3 mediates establishment of an antiviral state against hepatitis $\mathrm{C}$ virus in hepatoma cells. J Virol 83:9824-9834.

44. Cureton DK, Massol RH, Saffarian S, Kirchhausen TL, Whelan SP. 2009. Vesicular stomatitis virus enters cells through vesicles incompletely coated with clathrin that depend upon actin for internalization. PLoS Pathog 5:e1000394.

45. Bao X, Liu T, Shan Y, Li K, Garofalo RP, Casola A. 2008. Human metapneumovirus glycoprotein G inhibits innate immune responses. PLoS Pathog 4:e1000077.

46. Liu S, Qiu C, Miao R, Zhou J, Lee A, Liu B, Lester SN, Fu W, Zhu L, Zhang L, Xu J, Fan D, Li K, Fu M, Wang T. 2013. MCPIP1 restricts HIV infection and is rapidly degraded in activated CD4+ T cells. Proc Natl Acad Sci U S A 110:19083-19088.

47. Elbahesh H, Cline T, Baranovich T, Govorkova EA, Schultz-Cherry S, Russell CJ. 2014. Novel roles of focal adhesion kinase in cytoplasmic entry and replication of influenza A viruses. J Virol 88:6714-6728. 
48. Balish AL, Katz JM, Klimov AI. 2013. Influenza: propagation, quantification, and storage. Curr Protoc Microbiol Chapter 15:Unit15G.1. doi:

10.1002/9780471729259.mc15g01s29.

49. Zhou Z, Wang N, Woodson SE, Dong Q, Wang J, Liang Y, Rijnbrand R, Wei L, Nichols JE, Guo JT, Holbrook MR, Lemon SM, Li K. 2011. Antiviral activities of ISG20 in positive-strand RNA virus infections. Virology. 409:175-188.

50. Li K, Chen Z, Kato N, Gale M Jr, Lemon SM. 2005. Distinct poly(I-C) and virusactivated signaling pathways leading to interferon-beta production in hepatocytes. $\mathrm{J}$ Biol Chem 280:16739-16747.

51. Wang N, Dong Q, Li J, Jangra RK, Fan M, Brasier AR, Lemon SM, Pfeffer LM, Li K. 2010. Viral induction of the zinc-finger antiviral protein is IRF3-dependent but NF-kappa B-independent. J Biol Chem 285:6080-6090.

52. van Elden LJ, van Loon AM, van Alphen F, Hendriksen KA, Hoepelman AI, van Kraaij MG, Oosterheert JJ, Schipper P, Schuurman R, Nijhuis M. 2004. Frequent detection of human coronaviruses in clinical specimens from patients with respiratory tract infection by use of a novel real-time reverse-transcriptase polymerase chain reaction. J Infect Dis 189:652-657.

53. Chen G, Liu CH, Zhou L, Krug RM. 2014. Cellular DDX21 RNA helicase inhibits influenza A virus replication but is counteracted by the viral NS1 protein. Cell Host Microbe 15:484-493.

54. Marklund JK, Ye Q, Dong J, Tao YJ, Krug RM. 2012. Sequence in the influenza A virus nucleoprotein required for viral polymerase binding and RNA synthesis. J Virol 86:7292-7297.

55. Taylor RT, Lubick KJ, Robertson SJ, Broughton JP, Bloom ME, Bresnahan WA, Best SM. 2011. TRIM79 $\alpha$, an interferon-stimulated gene product, restricts tick-borne encephalitis virus replication by degrading the viral RNA polymerase. Cell Host Microbe 10:185-196.

56. Heinz FX, Stiasny K. 2012. Flaviviruses and flavivirus vaccines. Vaccine 30:43014306.

57. Wang RY, Li K. 2012. Host Factors in the Replication of Positive-Strand RNA Viruses. Chang Gung Med J 35:111-124.

58. Tsuchida T, Zou J, Saitoh T, Kumar H, Abe T, Matsuura Y, Kawai T, Akira S. 2010. The ubiquitin ligase TRIM56 regulates innate immune responses to intracellular double-stranded DNA. Immunity 33:765-776. 
59. Keskinen P, Nyqvist M, Sareneva T, Pirhonen J, Melén K, Julkunen I. 1999. Impaired antiviral response in human hepatoma cells. Virology 263:364-375.

60. Jiang D, Guo H, Xu C, Chang J, Gu B, Wang L, Block TM, Guo JT. 2008. Identification of three interferon-inducible cellular enzymes that inhibit the replication of hepatitis C virus. J Virol 82:1665-1678.

61. de Groot RJ, Baker SC, Baric RS, Brown CS, Drosten C, Enjuanes L, Fouchier RA, Galiano M, Gorbalenya AE, Memish ZA, Perlman S, Poon LL, Snijder EJ,Stephens GM, Woo PC, Zaki AM, Zambon M, Ziebuhr J. 2013. Middle East respiratory syndrome coronavirus (MERS-CoV): announcement of the Coronavirus Study Group. J Virol 87:7790-7792.

62. Barber GN. 2011. Innate immune DNA sensing pathways: STING, AIMII and the regulation of interferon production and inflammatory responses. Curr Opin Immunol 23:10-20.

63. Cai X, Chiu YH, Chen ZJ. 2014. The cGAS-cGAMP-STING Pathway of Cytosolic DNA Sensing and Signaling. Mol Cell 54:289-296.

64. Uchil PD, Quinlan BD, Chan WT, Luna JM, Mothes W. 2008. TRIM E3 ligases interfere with early and late stages of the retroviral life cycle. PLoS Pathog 4:e16.

65. Napolitano LM, Jaffray EG, Hay RT, Meroni G. 2011. Functional interactions between ubiquitin E2 enzymes and TRIM proteins. Biochem J 434:309-319.

66. Sanchez JG, Okreglicka K, Chandrasekaran V, Welker JM, Sundquist WI, Pornillos O. 2014. The tripartite motif coiled-coil is an elongated antiparallel hairpin dimer. Proc Natl Acad Sci U S A 111:2494-2499.

67. Hatakeyama S. 2011. TRIM proteins and cancer. Nat Rev Cancer 11:792-804.

68. Loedige I, Filipowicz W. 2009. TRIM-NHL proteins take on miRNA regulation. Cell 136:818-820.

69. Kano S, Miyajima N, Fukuda S, Hatakeyama S. 2008. Tripartite motif protein 32 facilitates cell growth and migration via degradation of Abl-interactor 2. Cancer Res 68:5572-5580.

70. Chase G, Deng T, Fodor E, Leung BW, Mayer D, Schwemmle M, Brownlee G. 2008. Hsp90 inhibitors reduce influenza virus replication in cell culture. Virology 377:431439.

71. Chou YY, Heaton NS, Gao Q, Palese P, Singer RH, Lionnet T. 2013. Colocalization of different influenza viral RNA segments in the cytoplasm before viral budding as shown by single-molecule sensitivity FISH analysis. PLoS Pathog 9:e1003358. 
72. Gack MU, Albrecht RA, Urano T, Inn KS, Huang IC, Carnero E, Farzan M, Inoue S, Jung JU, García-Sastre A. 2009. Influenza A virus NS1 targets the ubiquitin ligase TRIM25 to evade recognition by the host viral RNA sensor RIG-I. Cell Host Microbe 5:439-449.

73. Mäkelä SM, Österlund P, Westenius V, Latvala S, Diamond MS, Gale M Jr, Julkunen I. 2015. RIG-I signaling is essential for influenza B virus-induced rapid interferon gene expression. J Virol 89:12014-12025.

74. Fu B, Wang L, Ding H, Schwamborn JC, Li S, Dorf ME. 2015. TRIM32 senses and restricts Influenza A virus by ubiquitination of PB1 polymerase. PLoS Pathog 11:e1004960.

75. Edwards TA, Wilkinson BD, Wharton RP, Aggarwal AK. 2003. Model of the brain tumor-Pumilio translation repressor complex. Genes Dev 17:2508-2513.

76. Slack FJ, Ruvkun G. 1998. A novel repeat domain that is often associated with RING finger and B-box motifs. Trends Biochem Sci 23:474-475.

77. Chan YK, Gack MU. 2015. RIG-I-like receptor regulation in virus infection and immunity. Curr Opin Virol 12:7-14.

78. Mertins P, Qiao JW, Patel J, Udeshi ND, Clauser KR, Mani DR, Burgess MW, Gillette MA, Jaffe JD, Carr SA. 2013. Integrated proteomic analysis of posttranslational modifications by serial enrichment. Nat Methods 10:634-637.

79. Weber C, Schreiber TB, Daub H. 2012. Dual phosphoproteomics and chemical proteomics analysis of erlotinib and gefitinib interference in acute myeloid leukemia cells. J Proteomics 75: 1343-1356.

80. Schreiber TB, Mäusbacher N, Kéri G, Cox J, Daub H. 2010. An integrated phosphoproteomics work flow reveals extensive network regulation in earlylysophosphatidic acid signaling. Mol Cell Proteomics 9:1047-1062.

81. Sharma K, D'Souza RC, Tyanova S, Schaab C, Wiśniewski JR, Cox J, Mann M. 2014. Ultradeep human phosphoproteome reveals a distinct regulatory nature of Tyr and Ser/Thr-basedsignaling. Cell Rep 8:1583-1594.

82. Stuart SA, Houel S, Lee T, Wang N, Old WM, Ahn NG. 2015. A Phosphoproteomic Comparison of B-RAFV600E and MKK1/2 Inhibitors in Melanoma Cells. Mol Cell Proteomics 14:1599-1615.

83. Imami K, Sugiyama N, Kyono Y, Tomita M, Ishihama Y. 2008. Automated phosphoproteome analysis for cultured cancer cells by two-dimensional nanoLC-MS using a calcined titania/C18 biphasic column. Anal Sci 24:161-166. 
84. Li K, Li NL, Wei D, Pfeffer SR, Fan M, Pfeffer LM. 2012. Activation of chemokine and inflammatory cytokine response in hepatitis $\mathrm{C}$ virus-infected hepatocytes depends on Toll-like receptor 3 sensing of hepatitis $\mathrm{C}$ virus double-stranded RNA intermediates. Hepatology 55:666-675.

85. Stacey KB, Breen E, Jefferies CA. 2012. Tyrosine phosphorylation of the E3 ubiquitin ligase TRIM21 positively regulates interaction with IRF3 and henceTRIM21 activity. PLoS One $7:$ e34041.

86. Fletcher AJ, Mallery DL, Watkinson RE, Dickson CF, James LC. 2015. Sequential ubiquitination and deubiquitination enzymes synchronize the dual sensor and effector functions of TRIM21. Proc Natl Acad Sci U S A 112:10014-10019.

87. Liang Q, Deng H, Li X, Wu X, Tang Q, Chang TH, Peng H, Rauscher FJ 3rd, Ozato $\mathrm{K}$, Zhu F. 2011. Tripartite motif-containing protein 28 is a small ubiquitin-related modifier E3 ligase and negative regulator of IFN regulatory factor 7. J Immunol $187: 4754-4763$.

88. Zou W, Zhang DE. 2006. The interferon-inducible ubiquitin-protein isopeptide ligase (E3) EFP also functions as an ISG15 E3 ligase. J Biol Chem 281:3989-3994.

89. Shaw ML1, Stone KL, Colangelo CM, Gulcicek EE, Palese P. 2008. Cellular proteins in influenza virus particles. PLoS Pathog 4:e1000085.

90. Loedige I, Gaidatzis D, Sack R, Meister G, Filipowicz W. 2013. The mammalian TRIM-NHL protein TRIM71/LIN-41 is a repressor of mRNA function. Nucleic Acids Res 41:518-532. 


\section{VITA}

Baoming Liu was born in 1982 in Tianjin, China. He graduated with his Bachelor of Medicine degree from Tianjin Medical University in 2006 with a major in Laboratory Medicine. After obtaining a Master of Science degree in 2009 from Peking University Health Science Center, Baoming Liu joined Dr. Kui Li's laboratory at the University of Tennessee Health Science Center (UTHSC) to pursue basic scientific research training in virology and molecular biology as a Research Scholar. In 2012 he entered the Ph.D. program in Integrated Biomedical Sciences at UTHSC. During his doctoral dissertation research at Dr. Li's laboratory, Baoming Liu was studying the novel roles of TRIM56 in antiviral innate immunity. He graduated with the degree Doctor of Philosophy in 2016 with a focus on molecular virology and immunology. 